\title{
Lens space surgeries along certain 2-component links related with Park's rational blow down, and Reidemeister-Turaev torsion
}

\author{
Teruhisa KADOKAMI and Yuichi YAMADA
}

April 19, 2012

\begin{abstract}
We study lens space surgeries along two different families of 2-component links, denoted by $A_{m, n}$ and $B_{p, q}$, related with the rational homology 4-ball used in J. Park's (generalized) rational blow down. We determine which coefficient $r$ of the knotted component of the link yields a lens space by Dehn surgery. The link $A_{m, n}$ yields a lens space only by the known surgery with $r=m n$ and unexpectedly with $r=7$ for $(m, n)=(2,3)$. On the other hand, $B_{p, q}$ yields a lens space by infinitely many $r$. Our main tool for the proof is the Reidemeister-Turaev torsions, i.e. Reidemeister torsions with combinatorial Euler structures. Our results can be extended to the links whose Alexander polynomials are same with those of $A_{m, n}$ and $B_{p, q}$.
\end{abstract}

\section{Contents}

1 Introduction $\quad 2$

2 Reidemeister torsion $\quad 6$

2.1 Surgery formulae . . . . . . . . . . . . . . . . . 6

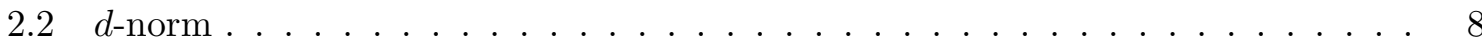

2.3 Combinatorial Euler structure (Reidemeister-Turaev torsion) $\ldots \ldots \ldots$

3 Alexander polynomial of $A_{m, n}$

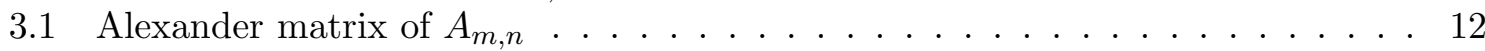

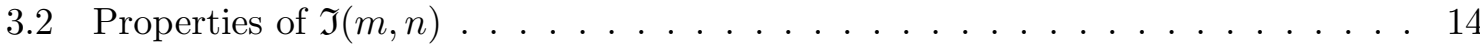

3.3 Proof of Theorem 3.2 (Alexander polynomial of $\left.A_{m, n}\right) \ldots \ldots \ldots$

4 Reidemeister torsions of $\left(A_{m, n} ; r, 0\right)$ and key lemmas $\quad 18$

4.1 The first homology of $\left(A_{m, n} ; r, 0\right) \ldots \ldots \ldots \ldots \ldots \ldots \ldots \ldots \ldots \ldots \ldots$

4.2 Reidemeister torsion of $\left(A_{m, n} ; r, 0\right) \ldots \ldots \ldots \ldots \ldots \ldots$

4.3 Necessary conditions . . . . . . . . . . . . . . . . . . . 24

\footnotetext{
${ }^{0} 2010$ Mathematics Subject Classification: 57M25, 57M27, 11R04 (primary), 57M50,11R27 (secondary).

Keywords: Dehn surgery, Alexander polynomial, Reidemeister torsion, combinatorial Euler structure, generalized rational blow down.

${ }^{1}$ The first author was supported by a grant (No.10801021/a010402) of NSFC. The second author was supported by KAKENHI (Grant-in-Aid for Scientific Research) No.21540072.
} 
5 Proof of Theorem 1.4 (Lens space surgeries along $A_{m, n}$ ) 27

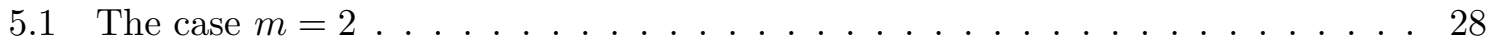

5.2 The case $n=m+1 \quad \ldots \ldots \ldots \ldots \ldots$

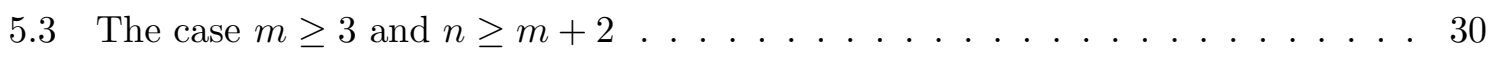

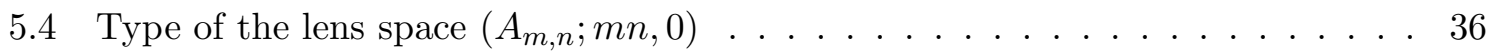

5.5 Type of the lens space $\left(A_{2,3} ; 7,0\right) \ldots \ldots \ldots \ldots \ldots$

6 Proof of Theorem 1.5 (Lens space surgeries along $B_{p, q}$ ) 38

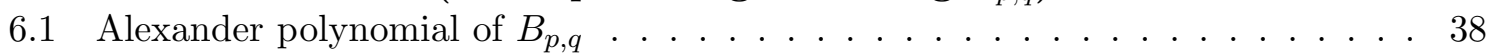

6.2 Conditions from the first homology of $\left(B_{p, q} ; r, 0\right) \ldots \ldots \ldots \ldots \ldots$

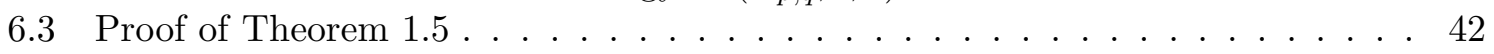

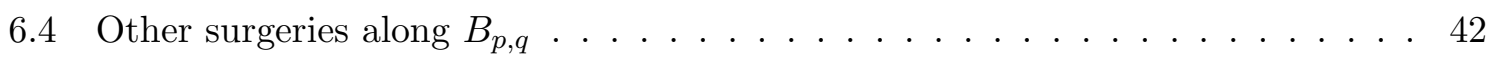

7 Lens space surgeries along $A_{m, n}$ other than $(r, 0)$-surgery 43

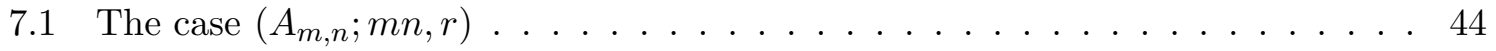

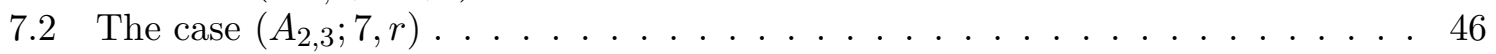

8 Final remarks $\quad 46$

8.1 Alternative proof for Theorem $1.4(1) \ldots \ldots \ldots \ldots \ldots$. . . . . . . . . . . . . . . . . . . . . .

8.2 Algebraic generalization ........................... 47

\section{Introduction}

For a coprime pair of non-zero positive integers $(m, n)$, let $A_{m, n}$ be a 2-component link in $S^{3}$ in Figure 1, where $K_{1}$ is the $(m, n)$-torus knot $T_{m, n}$ and $K_{2}$ is an unknot. The linking number of $K_{1}$ and $K_{2}$ is $m+n$. Next, for a coprime pair of non-zero integers $(p, q)$, let $B_{p, q}$ be a 2-component link in $S^{3}$ in Figure 2, where $K_{1}$ is the closure of the $(p, q)$-torus braid (the standard $p$-braid of the $(p, q)$-torus knot $\left.T_{p, q}\right)$ and $K_{2}$ is the braid axis. The linking number of $K_{1}$ and $K_{2}$ is $p$. For a $\mu$-component link $L=K_{1} \cup K_{2} \cup \cdots \cup K_{\mu}$, by $\left(L ; r_{1}, r_{2}, \ldots, r_{\mu}\right)$, we

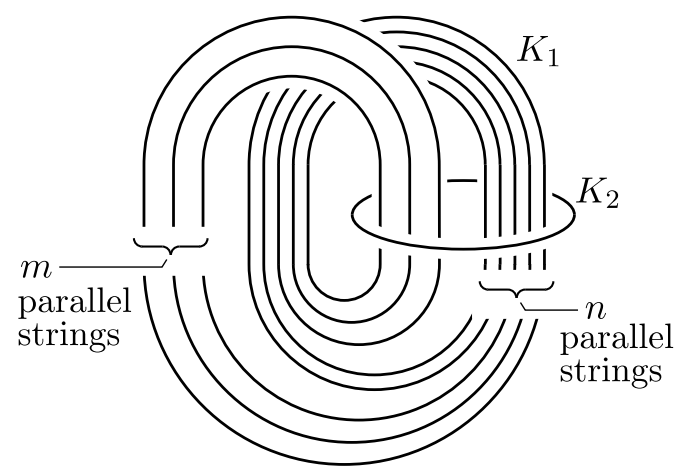

Figure 1: $A_{m, n} \quad($ ex. $(m, n)=(3,5))$

denote the result of $\left(r_{1}, r_{2}, \ldots, r_{\mu}\right)$-surgery along $L$, where $r_{i} \in \mathbb{Q} \cup\{\infty, \emptyset\}(i=1,2, \ldots, \mu)$. A Dehn surgery along a link is called a lens space surgery if the result is a lens space. We study lens space surgeries along the links $A_{m, n}$ and $B_{p, q}$, fixing the surgery coefficient of $K_{2}$ as 0 , 


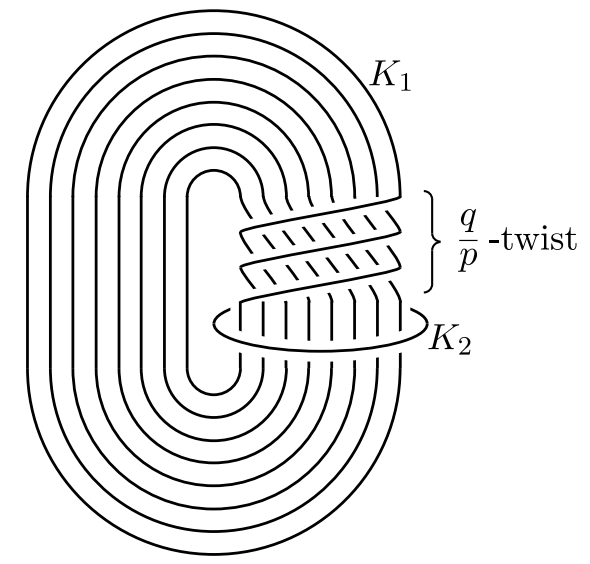

Figure 2: $B_{p, q}(\operatorname{ex} .(p, q)=(8,3))$

except in Section 7. Our convention on lens spaces is " $L(a, b)$ is the result of $-a / b$-surgery along the trivial knot".

The result of $(r, 0)$-surgery along a 2-component link $L=K_{1} \cup K_{2}$ in $S^{3}$ (as $\partial B^{4}$ ) such that $K_{2}$ is an unknot and $r \in \mathbb{Z}$ bounds a 4-manifold by attaching a 1-handle along $K_{2}$, and a 2-handle along $K_{1}$ with a framing $r$, to a 0 -handle $B^{4}$ along $S^{3}$. We denote the 4-manifold by $W^{4}(L ; r, \dot{0})$ following S. Akbulut [Ak] (see also [GS], [Kir]). Then $\pi_{1}\left(W^{4}(L ; r, \dot{0})\right) \cong \mathbb{Z} /|l| \mathbb{Z}$ and $H_{1}\left(\partial W^{4}(L ; r, \dot{0}) ; \mathbb{Z}\right) \cong \mathbb{Z} /|l|^{2} \mathbb{Z}$, where $l$ is the linking number of $K_{1}$ and $K_{2}$, and $W^{4}(L ; r, \dot{0})$ is a rational homology 4 -ball if and only if $l \neq 0$. We also note that $K_{1}$ can be regarded as a knot in $S^{1} \times S^{2}$.

We explain a background of our targets $A_{m, n}$ and $B_{p, q}$. J. Park [Pa] discussed generalized rational blow down, which is an operation on a 4-manifold cutting a certain submanifold $C_{p, q}$ and pasting a 4-manifold $W_{p, q}$ along $\partial C_{p, q} \cong \partial W_{p, q}$. The 4-manifold $W_{p, q}$ is a rational homology 4-ball that is characterized by $\pi_{1}\left(W_{p, q}\right) \cong \mathbb{Z} / p \mathbb{Z}$ and $\partial W_{p, q} \cong L\left(p^{2}, p q-1\right)$ (cf. $\left[\mathrm{FS},[\mathrm{CH}]\right.$ ). As far as the authors' knowledge, uniqueness of $W_{p, q}$ is not known well. A lens space surgery along $B_{p, q}$ whose result is $L\left(p^{2}, p q-1\right)$ is often used to describe $W_{p, q}$ (cf. [Li]). On the other hand, the second author [Yam3 found a lens space surgery along $A_{m, n}$ whose result is $L\left(p^{2}, p q-1\right)$ and defined an algorithm to determine $(m, n)$ from $(p, q)$ using the Euclidean algorithm. We also remark that the link $A_{m, n}$ appears as a bi-product of StipsiczSzabó-Wahl's construction [SSW, Remark 7.1], see also Endo-Mark-Horn-Morris [EMM]. We compare the links $A_{m, n}$ and $B_{p, q}$ by studying lens space surgeries along them. Our problem is:

Problem 1.1 When is $\left(A_{m, n} ; r, 0\right)\left(\left(B_{p, q} ; r, 0\right)\right.$, respectively) a lens space?

There are some trivial and overlapping cases: $B_{1, q}$ is the Hopf link and $A_{1, n}=B_{n+1,1}$. The links have some symmetries: $A_{m, n}=A_{n, m}$ and $B_{p,-q}$ is the mirror image of $B_{p, q}$. Throughout the paper, we assume the following:

Assumption For $A_{m, n}, \operatorname{gcd}(m, n)=1$ and $2 \leq m<n$. For $B_{p, q}, \operatorname{gcd}(p, q)=1, p \geq 2$ and $q \geq 1$.

The following theorem asserts that the link $A_{m, n}$ has at least one lens space surgery. 
Theorem 1.2 ([Yam3, Theorem 1.1]) For a pair $(m, n)$ satisfying the assumption, there exists a pair $(p, q)$ satisfying the assumption such that $\left(A_{m, n} ; m n, 0\right) \cong L\left(p^{2}, p q-1\right)$.

Notation 1.3 For integers $x$ and $N$, we denote the multiple inverse of $x$ modulo $N$ by $\bar{x}(\bmod N)$, i.e. $x \bar{x} \equiv 1(\bmod N)$. Note that, for a divisor $d(\geq 2)$ of $N$, both $x(\bmod d)$ and $\bar{x}(\bmod d)$ are uniquely determined by $x(\bmod N)$. We also use $\bar{x}$ as a representing integer of $\bar{x}(\bmod N)$.

Our first main theorem is the answer to Problem 1.1 for the link $A_{m, n}$.

Theorem 1.4 We assume $r \in \mathbb{Q}$.

(1) The result of $(r, 0)$-surgery along $A_{m, n}$ is a lens space if and only if

(i) $r=m n$, or

(ii) $r=7$ for $(m, n)=(2,3)$.

(2) The resulting lens spaces are as follows:

(i) $\left(A_{m, n} ; m n, 0\right) \cong L\left((m+n)^{2}, m \bar{n}\right)$, where $n \bar{n} \equiv 1\left(\bmod (m+n)^{2}\right)$, and

(ii) $\left(A_{2,3} ; 7,0\right) \cong L(25,7)$.

The "if part" of Theorem 1.4 (1) (i) follows from Theorem 1.2, Thus our purpose is to show the "only if part" of (1), and (2).

Our second main theorem is the answer to Problem 1.1 for the link $B_{p, q}$, which is contrast to $A_{m, n}$.

Theorem 1.5 We set $\alpha / \beta \in \mathbb{Q}$, where $\alpha$ and $\beta$ are coprime integers.

(1) The result of $(\alpha / \beta, 0)$-surgery along $B_{p, q}$ is a lens space if and only if $|\alpha-p q \beta|=1$.

(2) For $\alpha / \beta$ with $|\alpha-p q \beta|=1$, the resulting lens space is $\left(B_{p, q} ; \alpha / \beta, 0\right) \cong L\left(p^{2} \beta, \alpha\right)$.

Remark 1.6 We remark on surgeries along the mirror images of the links. Naturally, we have $B_{-p,-q}=B_{p, q}, B_{-p, q}=B_{p,-q}$ as unoriented links, and $B_{p,-q}$ is the mirror image of $B_{p, q}$. Theorem [1.5 can be extended to the cases $p<0$ or/and $q<0$. Similarly, Theorem 1.2 can be extended to the mirror image $A_{m, n}$ ! of $A_{m, n}$. We note that $A_{m, n}$ ! is not included in the family $\left\{A_{m, n}\right\}$.

We list some corollaries without the proofs.

Corollary 1.7 The lens space $\left(A_{2,3} ; 7,0\right) \cong L(25,7)$ cannot be obtained by any $(r, 0)$-surgery along $B_{p, q}$.

Corollary 1.8 (Integral lens space surgery along $B_{p, q}$ ) Suppose $\beta=1$ in Theorem 1.5 . Then $\left(B_{p, q} ; \alpha, 0\right)$ is a lens space if and only if $\alpha=p q-1$ or $p q+1$. The resulting lens space is $L\left(p^{2}, p q-1\right)$ or $L\left(p^{2}, p q+1\right)$, respectively.

By Theorem 1.2 and Corollary 1.8 , both $W^{4}\left(A_{m, n} ; m n, \dot{0}\right)$ and $W^{4}\left(B_{p, q} ; p q-1, \dot{0}\right)$ represent $W_{p, q}$, under the correspondence between $(m, n)$ and $(p, q)$ in Theorem 1.2, Our second problem is: 
Problem 1.9 Are $W^{4}\left(A_{m, n} ; m n, \dot{0}\right)$ and $W^{4}\left(B_{p, q} ; p q-1, \dot{0}\right)$ diffeomorphic, homeomorphic or homotopic relative to the boundaries?

The lens space $\left(A_{2,3} ; 7,0\right)$ in Corollary 1.7 satisfies $L(25,7) \cong-L(25,7)$. On the other hand, on the integral lens spaces surgeries along $B_{p, q}$ in Corollary 1.8, it is easy to see that $L\left(p^{2}, p q-1\right) \cong-L\left(p^{2}, p q+1\right)$ and that $L\left(p^{2}, p q-1\right) \neq L\left(p^{2}, p q+1\right)$. Thus we have

Corollary 1.10 Only in this corollary, we regard the link $A_{m, n}$ or $B_{p, q}$ as a knot $K_{1}$ in $S^{1} \times S^{2}$, the result of 0 -surgery along $K_{2}$. We assume that $2 \leq m<n, 2 \leq p$ and $1 \leq q$. Then, any knot $A_{m, n}$ is not isotopic to any knot $B_{p, q}$.

Corollary 1.10 asserts that the attaching parts $K_{1}$ of the 2-handles of $W^{4}\left(A_{m, n} ; m n, \dot{0}\right)$ and $W^{4}\left(B_{p, q} ; p q-1, \dot{0}\right)$ are not isotopic in $S^{1} \times S^{2}$. Thus, the handle decompositions of the rational homology 4-balls do not move to each other by only handle slides of the 2-handles over the 1-handles. Problem 1.9 may be still open.

Our results can be regarded as lens space surgeries along 2-component links. For usual lens space surgery along knots, see [Ba], [Ber], [CGLS], Go] and so on. We point out that Theorem 1.4 (on $\left.A_{m, n}\right)$ can also be obtained by the results " $\left(A_{m, n} ; \emptyset, 0\right)$ is a hyperbolic manifold" in [DMM1, and the Cyclic Surgery Theorem [CGLS]. If we use them, then the proof of the theorem can be shortened (see Subsection 8.1). The reason why we do not use them is to clarify effectivity of Alexander polynomials and Reidemeister-Turaev torsions from technical point of view. As consequences, they preserved information of lens space surgeries completely in the present case, and our results are generalized to wider situations, see Theorem 8.1 and Theorem 8.2. The links $A_{m, n}$ are related to subfamilies of knots, called TypeVII and Type VIII in Berge's list $\mathrm{Ber}$, see [Kad4, Yam1 and Yam2. On the other hand, the results $\left(B_{p, q} ; r, 0\right)$ with any $r$ is a Seifert manifold (or a graph manifold), thus Theorem 1.5 looks like L. Moser's result [Mos] on lens space surgeries along torus knots.

We also study a generalization of Theorem 1.4. To determine all Dehn surgeries along $A_{m, n}$ is a hard problem. As far as the authors' knowledge, the complete answer is not given. From our present results and some known results, we feel like to raise the following conjecture:

Conjecture 1.11 Let $M=\left(A_{m, n} ; \alpha_{1} / \beta_{1}, \alpha_{2} / \beta_{2}\right)$ be the result of $\left(\alpha_{1} / \beta_{1}, \alpha_{2} / \beta_{2}\right)$-surgery along $A_{m, n}$, where $\alpha_{i}$ and $\beta_{i}(i=1,2)$ are coprime integers with $\beta_{i} \geq 1$. Then $M$ is a lens space if and only if (1) $\alpha_{1} / \beta_{1}=m n$ and $\beta_{2}=1$, or (2) $\alpha_{1} / \beta_{1}=7$ for $(m, n)=(2,3)$.

K. Ichihara ([IS]) informed to the authors that, if we fix $\alpha_{1} / \beta_{1}=m n$, then $M$ is a lens space for any integer $\left(\beta_{2}=1\right)$. He says that it can be shown by a method in YYam3] (i.e. a geometric method). In Section 7 , we compute the Reidemeister torsions of $M$ under the case (1) $\alpha_{1} / \beta_{1}=m n$ and $(2) \alpha_{1} / \beta_{1}=7$ for $(m, n)=(2,3)$, respectively. A partial affirmative answer for "only if part" of the case (1) is given.

In Section 2, we explain about the Reidemeister torsion and its basic properties such as surgery formulae, $d$-norm and combinatorial Euler structure (i.e. Reidemeister-Turaev torsion). In Section 3, we compute the Alexander polynomial of $A_{m, n}$. In Section 4, we compute the Reidemeister torsions of $\left(A_{m, n} ; r, 0\right)$ by using the results in Section 2 and Section 3 , In Section 5, we prove the "only if part" of Theorem 1.4 (1) by using the Reidemeister-Turaev torsions, and Theorem 1.4 (2) by using the values of the Reidemeister torsions, Theorem 1.2, 
and Kirby moves. In Section [6, we prove Theorem 1.5 by using Seifert structures of the link complement and the Reidemeister torsions. In Section 7, we study some lens space surgeries along $A_{m, n}$ other than $(r, 0)$-surgery, related to Conjecture 1.11, In Section 8, we give an alternative proof of Theorem 1.4 (1) under some assumption, and we generalize Theorem 1.4 and Theorem 1.5 .

\section{Reidemeister torsion}

Our method to prove the main theorems is to deduce necessary conditions from the Reidemeister torsions of both the surgered manifolds and lens spaces. In this section, we state surgery formulae of the Reidemeister torsions (Subsection 2.1), and define derived invariants: one is the $d$-norm (Subsection 2.2), and the other is the Reidemeister-Turaev torsion which is a lift of the Reidemeister torsions by fixing a combinatorial Euler structure (Subsection 2.3).

\subsection{Surgery formulae}

For a precise definition of the Reidemeister torsion, the reader refer to V. Turaev [Tur1, Tur2]. Throughout this paper, we use the following notations.

Notation 2.1 (for manifolds and homologies) Let $L=K_{1} \cup \cdots \cup K_{\mu}$ be an oriented $\mu$-component link in a homology 3-sphere.

$\begin{array}{ll}E_{L} & \text { the complement of } L . \\ m_{i}, l_{i} & \text { a meridian and a longitude of the } i \text {-th component } K_{i} . \\ {\left[m_{i}\right],\left[l_{i}\right]} & \text { their homology classes. } \\ \Delta_{L}\left(t_{1}, \ldots, t_{\mu}\right) & \text { the Alexander polynomial of } L, \text { where } t_{i} \text { is represented by } m_{i} . \\ \left(L ; r_{1}, \ldots, r_{\mu}\right) & \text { the result of }\left(r_{1}, \ldots, r_{\mu}\right) \text {-surgery along } L, \\ \quad \text { where } r_{i} \in \mathbb{Q} \cup\{\infty, \emptyset\} \text { is the surgery coefficient of } K_{i} . \\ V_{i} \\ m_{i}^{\prime},\left[m_{i}^{\prime}\right] \quad \begin{array}{l}\text { the solid torus attached along } K_{i} \text { in the Dehn surgery. } \\ l_{i}^{\prime},\left[l_{i}^{\prime}\right]\end{array} \quad \text { an oridian of } V_{i}, \text { and its homology class. } \\ \end{array}$

Let $X$ be a finite CW complex and $\pi: \tilde{X} \rightarrow X$ its maximal abelian covering. Then $\tilde{X}$ has a CW structure induced by that of $X$ and $\pi$, and the cell chain complex $\mathbf{C}_{*}$ of $\tilde{X}$ has a $\mathbb{Z}[H]$-module structure, where $H=H_{1}(X ; \mathbb{Z})$ is the first homology of $X$. For an integral domain $R$ and a ring homomorphism $\psi: \mathbb{Z}[H] \rightarrow R$, "the chain complex of $\tilde{X}$ related with $\psi$ ", denoted by $\mathbf{C}_{*}^{\psi}$, is $\mathbf{C}_{*} \otimes_{\mathbb{Z}[H]} Q(R)$, where $Q(R)$ is the quotient field of $R$. The Reidemeister torsion of $X$ related with $\psi$, denoted by $\tau^{\psi}(X)$, is calculated from $\mathbf{C}_{*}^{\psi}$, and is an element of $Q(R)$ determined up to multiplication of $\pm \psi(h)(h \in H)$. If $R=\mathbb{Z}[H]$ and $\psi$ is the identity map, then we denote $\tau^{\psi}(X)$ by $\tau(X)$. We note that $\tau^{\psi}(X)$ is not zero if and only if $\mathbf{C}_{*}^{\psi}$ is acyclic.

Notation 2.2 (for algebra)

For a pair of elements $A, B$ in $Q(R)$, if there exists an element $h \in H$ such that $A= \pm \psi(h) B$, then we denote the equality by $A \doteq B$. We will often take a field $F$ and a ring homomorphism $\psi: \mathbb{Z}\left[H_{1}(M)\right] \rightarrow F$. We mainly use the d-th cyclotomic fields $\mathbb{Q}\left(\zeta_{d}\right)$ as $F$, where $\zeta_{d}$ is a primitive d-th root of unity. 
For the first lemma, we need a little general setting: Let $E$ be a compact 3-manifold whose boundary $\partial E$ consists of tori. We study the 3-manifold $M=E \cup V_{1} \cup \cdots \cup V_{n}$ obtained by attaching solid tori $V_{i}$ to $E$ by attaching maps $f_{i}: \partial V_{i} \rightarrow \partial E\left(\operatorname{Im}\left(f_{i}\right) \cap \operatorname{Im}\left(f_{j}\right)=\emptyset\right.$ for $\left.i \neq j\right)$. We let $\iota: E \hookrightarrow M$ denote the natural inclusion.

Lemma 2.3 (Surgery formula I) If $\psi\left(\left[l_{i}^{\prime}\right]\right) \neq 1$ for every $i=1, \ldots, n$, then we have

$$
\tau^{\psi}(M) \doteq \tau^{\psi^{\prime}}(E) \prod_{i=1}^{n}\left(\psi\left(\left[l_{i}^{\prime}\right]\right)-1\right)^{-1}
$$

where $\psi^{\prime}=\psi \circ \iota_{*}\left(\iota_{*}\right.$ is a ring homomorphism induced by $\left.\iota\right)$.

For the case of the link complement of a homology 3-sphere, the Reidemeister torsion is closely related with the Alexander polynomial.

Lemma 2.4 (Milnor Mil]) For a $\mu$-component link $L=K_{1} \cup \cdots \cup K_{\mu}$ in a homology 3-sphere, we have

$$
\tau\left(E_{L}\right) \doteq \begin{cases}\Delta_{L}\left(t_{1}\right)\left(t_{1}-1\right)^{-1} & (\mu=1) \\ \Delta_{L}\left(t_{1}, \ldots, t_{\mu}\right) & (\mu \geq 2) .\end{cases}
$$

By Lemma 2.1 and Lemma 2.2, we have the following:

Lemma 2.5 (Surgery formula II) (T. Sakai [Sa], V. G. Turaev [Tur1])

(1) Let $K$ be a knot in a homology 3-sphere. We set $M=(K ; p / q)(|p| \geq 2)$, where $p$ and $q$ are coprime integers. Let $T$ be a generator of $H_{1}(M)$ represented by a meridian of $K$, and $\psi_{d}: \mathbb{Z}\left[H_{1}(M)\right] \rightarrow \mathbb{Q}\left(\zeta_{d}\right)$ a ring homomorphism defined by $\psi_{d}(T)=\zeta_{d}$, where $d$ $(\geq 2)$ is a divisor of $p$. Then we have

$$
\tau^{\psi_{d}}(M) \doteq \Delta_{K}\left(\zeta_{d}\right)\left(\zeta_{d}-1\right)^{-1}\left(\zeta_{d}^{\bar{q}}-1\right)^{-1}
$$

where $q \bar{q} \equiv 1(\bmod p)$.

(2) Let $L$ be a $\mu$-component link in a homology 3 -sphere. We set $M=\left(L ; p_{1} / q_{1}, \ldots, p_{\mu} / q_{\mu}\right)(\mu \geq$ 2), where $p_{i}$ and $q_{i}$ are coprime integers for every $i=1, \ldots, \mu$. Let $F$ be a field, $\psi: \mathbb{Z}\left[H_{1}(M)\right] \rightarrow F$ a ring homomorphism with $\psi\left(\left[m_{i}\right]^{r_{i}}\left[l_{i}\right]^{s_{i}}\right) \neq 1$ for every $i=1, \ldots, \mu$, where $r_{i}$ and $s_{i}$ are integers satisfying $p_{i} s_{i}-q_{i} r_{i}=-1$. Then we have

$$
\tau^{\psi}(M) \doteq \Delta_{L}\left(\psi\left(\left[m_{1}\right]\right), \ldots, \psi\left(\left[m_{\mu}\right]\right)\right) \prod_{i=1}^{\mu}\left(\psi\left(\left[m_{i}\right]^{r_{i}}\left[l_{i}\right]^{s_{i}}\right)-1\right)^{-1} .
$$

Example 2.6 The lens space $L(p, q)$ is obtained as -p/q-surgery along the unknot. By Lemma 2.5 (1), for a divisor $d \geq 2$ of $p$, we have

$$
\tau^{\psi_{d}}(L(p, q)) \doteq\left(\zeta_{d}-1\right)^{-1}\left(\zeta_{d}^{\bar{q}}-1\right)^{-1},
$$

where $q \bar{q} \equiv 1(\bmod p)$.

We recall the Torres formula for the Alexander polynomials. 
Lemma 2.7 (Torres formula) ([Tor]) Let $L=K_{1} \cup \cdots \cup K_{\mu} \cup K_{\mu+1}(\mu \geq 1)$ be an oriented $(\mu+1)$-component link, $L^{\prime}=K_{1} \cup \cdots \cup K_{\mu}$ a $\mu$-component sublink, and $\ell_{i}=\operatorname{lk}\left(K_{i}, K_{\mu+1}\right)(i=$ $1, \ldots, \mu)$. Then we have

$$
\Delta_{L}\left(t_{1}, \ldots, t_{\mu}, 1\right) \doteq \begin{cases}\frac{t_{1}^{\ell}-1}{t_{1}-1} \Delta_{L^{\prime}}\left(t_{1}\right) & (\mu=1), \\ \left(t_{1}^{\ell_{1}} \cdots t_{\mu}^{\ell_{\mu}}-1\right) \Delta_{L^{\prime}}\left(t_{1}, \ldots, t_{\mu}\right) & (\mu \geq 2) .\end{cases}
$$

\section{$2.2 d$-norm}

About algebraic fields, the reader refer to L. C. Washington Was] for example.

For an element $x$ in the $d$-th cyclotomic field $\mathbb{Q}\left(\zeta_{d}\right)$, the $d$-norm of $x$ is defined as

$$
N_{d}(x)=\prod_{\sigma \in \operatorname{Gal}\left(\mathbb{Q}\left(\zeta_{d}\right) / \mathbb{Q}\right)} \sigma(x),
$$

where $\operatorname{Gal}\left(\mathbb{Q}\left(\zeta_{d}\right) / \mathbb{Q}\right)$ is the Galois group related with a Galois extension $\mathbb{Q}\left(\zeta_{d}\right)$ over $\mathbb{Q}$. The following is well-known.

\section{Proposition 2.8}

(1) If $x \in \mathbb{Q}\left(\zeta_{d}\right)$, then $N_{d}(x) \in \mathbb{Q}$. The map $N_{d}: \mathbb{Q}\left(\zeta_{d}\right) \backslash\{0\} \rightarrow \mathbb{Q} \backslash\{0\}$ is a group homomorphism.

(2) If $x \in \mathbb{Z}\left[\zeta_{d}\right]$, then $N_{d}(x) \in \mathbb{Z}$.

By easy calculations, we have the following.

\section{Lemma 2.9}

(1) $N_{d}\left( \pm \zeta_{d}\right)= \begin{cases} \pm 1 & (d=2) \\ 1 & (d \geq 3)\end{cases}$
(2) $N_{d}\left(1-\zeta_{d}\right)= \begin{cases}\ell & (d \text { is a power of a prime } \ell \geq 2), \\ 1 & \text { (otherwise }) .\end{cases}$

About applications of $d$-norms, for example, see [Kad1, Kad2, Kad3, KMS, KY1, KY2].

W. Franz $\mathrm{Fz}$ showed the following, and classified lens spaces by using it. We state a modified version (cf. [KY1]).

Lemma 2.10 (Franz $[\mathrm{Fz}]$ ) Let $p \geq 2$ be an integer, and $(\mathbb{Z} / p \mathbb{Z})^{\times}$the multiplicative group of a ring $\mathbb{Z} / p \mathbb{Z}$. For $a_{i}, b_{i} \in(\mathbb{Z} / p \mathbb{Z})^{\times}(i=1, \ldots, n)$, suppose

$$
\prod_{i=1}^{n}\left(\zeta_{p}^{a_{i}}-1\right) \doteq \prod_{i=1}^{n}\left(\zeta_{p}^{b_{i}}-1\right)
$$

where $\zeta_{p}$ is a primitive $p$-th root of unity. Then there exists a permutation $\sigma$ of $\{1, \ldots, n\}$ such that $a_{i}= \pm b_{\sigma(i)}$ for all $i=1, \ldots, n$. In other words, $\left\{ \pm a_{i}(\bmod p)\right\}=\left\{ \pm b_{i}(\bmod p)\right\}$ as multiple sets.

We will use this lemma in Lemma 4.9 and in Section 8 . 


\subsection{Combinatorial Euler structure (Reidemeister-Turaev torsion)}

Let $M$ be a homology lens space with $H=H_{1}(M) \cong \mathbb{Z} / p \mathbb{Z}(p \geq 2)$. Then the Reidemeister torsion $\tau^{\psi_{d}}(M)$ of $M$ related with $\psi_{d}$ is determined up to multiplication of $\pm \zeta_{d}^{m}(m \in \mathbb{Z})$, where $d \geq 2$ is a divisor of $p$ and $\psi_{d}$ is the same ring homomorphism as in Lemma 2.5 (1). Once we fix a basis of a cell chain complex for the maximal abelian covering of $M$ as a $\mathbb{Z}[H]=\mathbb{Z}\left[t, t^{-1}\right] /\left(t^{p}-1\right)$-module, the value $\tau^{\psi_{d}}(M)$ is uniquely determined as an element of $\mathbb{Q}\left(\zeta_{d}\right)$ for every $d$. The choice of the basis up to "base change equivalence" is called a combinatorial Euler structure of $M$ (cf. Turaev Tur2]). The Reidemeister torsion of a manifold with a fixed combinatorial Euler structure is said the Reidemeister-Turaev torsion.

We consider the sequence of the values $\tau^{\psi_{d}}(M)$ in $\mathbb{Q}\left(\zeta_{d}\right)$ of the Reidemeister-Turaev torsion for every divisor $d \geq 2$ of $p$, and regard them as a value sequence $\left\{\tau^{\psi_{d}}(M)\right\}_{d \mid p, d \geq 2}$ defined as below.

Definition 2.11 A sequence of values $\boldsymbol{x}=\left\{x_{d}\right\}_{d \mid p, d \geq 2}$ is a value sequence (of degree $p$ ) if $x_{d} \in \mathbb{Q}\left(\zeta_{d}\right)$ for every $d$. Two value sequences $\boldsymbol{x}=\left\{x_{d}\right\}_{d \mid p, d \geq 2}$ and $\boldsymbol{y}=\left\{y_{d}\right\}_{d \mid p, d \geq 2}$ are equal $(\boldsymbol{x}=\boldsymbol{y})$ if $x_{d}=y_{d}$ for every $d$. We are mainly concerned with the value sequence of type $\boldsymbol{x}=\left\{F\left(\zeta_{d}\right)\right\}_{d \mid p, d \geq 2}$ for a rational function $F(t) \in \mathbb{Q}(t)$. In such a case, we say that $\boldsymbol{x}$ is induced by $F(t)$ and that $F(t)$ is a lift of $\boldsymbol{x}$. A control of $\boldsymbol{x}=\left\{x_{d}\right\}_{d \mid p, d \geq 2}$ by a trivial unit $u=\eta t^{m} \in \mathbb{Q}\left[t, t^{-1}\right] /\left(t^{p}-1\right)$ is defined by

$$
u \boldsymbol{x}=\left\{\eta \zeta_{d}^{m} x_{d}\right\}_{d \mid p, d \geq 2},
$$

where $\eta=1$ or -1 (constant) and $m \in \mathbb{Z}$. Two value sequences $\boldsymbol{x}=\left\{x_{d}\right\}_{d \mid p, d \geq 2}$ and $\boldsymbol{y}=$ $\left\{y_{d}\right\}_{d \mid p, d \geq 2}$ are control equivalent if there is a trivial unit $u \in \mathbb{Q}\left[t, t^{-1}\right] /\left(t^{p}-1\right)$ such that $\boldsymbol{y}=u \boldsymbol{x}$. A value sequence $\boldsymbol{x}=\left\{x_{d}\right\}_{d \mid p, d \geq 2}$ is a real value sequence if $x_{d}$ is a real number for every $d$.

Let $M$ be a homology lens space with $H_{1}(M) \cong \mathbb{Z} / p \mathbb{Z}(p \geq 2)$. Then a sequence $\left\{\tau^{\psi_{d}}(M)\right\}_{d \mid p, d \geq 2}$ of the Reidemeister torsions of $M$ with a combinatorial Euler structure is a value sequence of degree $p$. We say the value sequence a torsion sequence of $M$.

\section{Lemma 2.12}

(1) Let $M$ and $M^{\prime}$ be homeomorphic homology lens spaces with $H_{1}(M) \cong H_{1}\left(M^{\prime}\right) \cong$ $\mathbb{Z} / p \mathbb{Z}(p \geq 2)$. Then torsion sequences $\left\{\tau^{\psi_{d}}(M)\right\}_{d \mid p, d \geq 2}$ and $\left\{\tau^{\psi_{d}^{\prime}}\left(M^{\prime}\right)\right\}_{d \mid p, d \geq 2}$ related with the corresponding representations $\psi_{d}$ and $\psi_{d}^{\prime}$ (i.e., $\psi_{d}=\psi_{d}^{\prime} \circ h_{*}$, where $h_{*}$ is the induced homomorphism of the homeomorphism) are control equivalent.

(2) Let $M$ be a homology lens space with $H_{1}(M) \cong \mathbb{Z} / p \mathbb{Z}(p \geq 2)$. Then we can control a torsion sequence of $M$ into a real value sequence.

Proof (1) It is easy to see.

(2) Here we let $\zeta$ denote any $d$-th primitive root $\left(\zeta_{d}\right)$ of unity. Since $M$ is obtained by $p / q$ surgery along a knot $K$ in a homology 3 -sphere for some $q$ (cf. [BL]). By Lemma 2.5 (1), we have

$$
\tau^{\psi_{d}}(M) \doteq \Delta_{K}(\zeta)(\zeta-1)^{-1}\left(\zeta^{\bar{q}}-1\right)^{-1}
$$


where $q \bar{q} \equiv 1(\bmod p)$. By the duality of the Alexander polynomial (cf. [Mil, Tur1, Tur2]), we may assume

$$
\Delta_{K}(t)=\Delta_{K}\left(t^{-1}\right) .
$$

This is also a control of the combinatorial Euler structure of the exterior of $K$, which induces a control of a torsion sequence of $M$. We take an odd integer lift of $\bar{q}$. Then

$$
\zeta^{\frac{1+\bar{q}}{2}} \Delta_{K}(\zeta)(\zeta-1)^{-1}\left(\zeta^{\bar{q}}-1\right)^{-1}
$$

is a real number for every $d$.

Lemma 2.13 If two real value sequences $\boldsymbol{x}=\left\{x_{d}\right\}_{d \mid p, d \geq 2}$ and $\boldsymbol{y}=\left\{y_{d}\right\}_{d \mid p, d \geq 2}$ of degree $p$ are control equivalent satisfying $\boldsymbol{y}=u \boldsymbol{x}$ for a trivial unit $u=\eta t^{m} \in \mathbb{Z}\left[t, t^{-1}\right] /\left(t^{p}-1\right)$, where $\eta= \pm 1$ and $m \in \mathbb{Z}$, then the possibility of $u$ is restricted as follows:

(i) If $p$ is odd, then $u=1$ or -1 .

(ii) If $p$ is even, then $u=1,-1, t^{p / 2}$ or $-t^{p / 2}$.

Proof Since the ratio $\zeta_{p}^{m}= \pm y_{p} / x_{p}$ is a real number, we have (i) $m \equiv 0(\bmod p)$ if $p$ is odd, and (ii) $m \equiv 0$ or $p / 2(\bmod p)$ if $p$ is even.

Definition 2.14 (Symmetric Laurent polynomial) A Laurent polynomial $F(t) \in \mathbb{Q}\left[t, t^{-1}\right]$ is symmetric if it is of the form

$$
F(t)=a_{0}+\sum_{i=1}^{\infty} a_{i}\left(t^{i}+t^{-i}\right),
$$

where $a_{i}$ is a rational number for all $i=1,2, \ldots$ and $a_{i}=0$ for every sufficiently large $i$. Note that, if $F(t)$ is a symmetric Laurent polynomial, the induced value sequence $\left\{F\left(\zeta_{d}\right)\right\}_{d \mid p, d \geq 2}$ is a real value sequence. We are concerned with symmetric Laurent polynomials that are lifts (in $\mathbb{Q}\left[t, t^{-1}\right]$ ) of a polynomial in the quotient ring $\mathbb{Q}\left[t, t^{-1}\right] /\left(t^{p}-1\right)$. We say that $F(t)$ (as above) is reduced if $a_{i}=0$ for all $i>[p / 2]$. We often reduce the symmetric polynomials by using $t^{i}+t^{-i}=t^{p+i}+t^{-(p+i)}$ modulo $\left(t^{p}-1\right)$. We let red $(F(t))$ denote the reduction of $F(t)$ (i.e., $\operatorname{red}(F(t))$ is reduced and $\operatorname{red}(F(t))=F(t)$ in $\left.\mathbb{Q}\left[t, t^{-1}\right] /\left(t^{p}-1\right)\right)$.

For a Laurent polynomial $F(t) \in \mathbb{Q}\left[t, t^{-1}\right]$, the span of $F(t)$ is the difference of the maximal degree and the minimal degree of $F(t)$, and we denote it by $\operatorname{span}(F(t))$. Note that the span of a symmetric Laurent polynomial is always even, and that the span of a reduced symmetric Laurent polynomial is less than or equal to $2[p / 2]$.

Lemma 2.15 Let $N \geq 2$ be an integer. Let $F(t), G(t)$ be symmetric Laurent polynomials and $\boldsymbol{x}=\left\{F\left(\zeta_{d}\right)\right\}_{d \mid N, d \geq 2}, \boldsymbol{y}=\left\{G\left(\zeta_{d}\right)\right\}_{d \mid N, d \geq 2}$ the induced real value sequences, respectively. If $\boldsymbol{x}$ and $\boldsymbol{y}$ are control equivalent, i.e., $u \boldsymbol{x}=\boldsymbol{y}$ for a trivial unit $u$ (here, $u=1$ or -1 if $N$ is odd, $u=1,-1, t^{N / 2}$ or $-t^{N / 2}$ if $N$ is even, by Lemma 2.13), then we have a congruence

$$
u F(t) \equiv G(t) \quad \bmod t^{N-1}+t^{N-2}+\cdots+t+1 .
$$

Furthermore, assuming $\operatorname{span}(G(t)) \leq 2([N / 2]-1)$, 
(i) In the case that $u=1$ or -1 and $\operatorname{span}(F(t)) \leq N-2$, we have an identity $u F(t)=G(t)$ in $\mathbb{Q}\left[t, t^{-1}\right]$.

(ii) Otherwise (in the case that $N$ is even and $u=\eta t^{N / 2}$ with $\eta=1$ or -1 ), if $\operatorname{span}\left(\operatorname{red}\left(t^{N / 2} F(t)\right)\right) \leq$ $N-2$, then we have $\operatorname{red}\left(t^{N / 2} F(t)\right)=\eta G(t)$ in $\mathbb{Q}\left[t, t^{-1}\right]$.

Proof By the Chinese Remainder Theorem, we have a ring isomorphism:

$$
\mathbb{Q}\left[t, t^{-1}\right] /\left(t^{N-1}+t^{N-2}+\cdots+t+1\right) \cong \bigoplus_{d \mid N, d \geq 2} \mathbb{Q}\left(\zeta_{d}\right)
$$

where $f(t)$ in the left-hand side maps to the value sequences $\left\{f\left(\zeta_{d}\right)\right\}_{d \mid N, d \geq 2}$ in the right-hand side. The isomorphism implies the required congruence.

Since $2([N / 2]-1)<N-1=\operatorname{span}\left(t^{N-1}+t^{N-2}+\cdots+t+1\right)$, we have the identities.

Lemma 2.16 Let $N \geq 2$ be an integer. Let $F(t), G(t)$ be symmetric Laurent polynomials and $\boldsymbol{x}=\left\{F\left(\zeta_{d}\right)\right\}_{d \mid N, d \geq 2}, \boldsymbol{y}=\left\{G\left(\zeta_{d}\right)\right\}_{d \mid N, d \geq 2}$ the induced real value sequences, respectively. If $\boldsymbol{x}$ and $\boldsymbol{y}$ are control equivalent, i.e., $u \boldsymbol{x}=\boldsymbol{y}$ for a trivial unit $u$ (here, $u=1$ or -1 if $N$ is odd, $u=1,-1, t^{N / 2}$ or $-t^{N / 2}$ if $N$ is even, by Lemma 2.13), and $F(1)=G(1)=0$, then we have a congruence

$$
u F(t) \equiv G(t) \quad \bmod t^{N}-1 .
$$

Furthermore, assuming $\operatorname{span}(G(t)) \leq 2[N / 2]$,

(i) In the case that $u=1$ or -1 and $\operatorname{span}(F(t)) \leq N-1$, we have an identity $u F(t)=G(t)$ in $\mathbb{Q}\left[t, t^{-1}\right]$.

(ii) Otherwise (in the case that $N$ is even and $u=\eta t^{N / 2}$ with $\eta=1$ or -1 ), we have $\operatorname{red}\left(t^{N / 2} F(t)\right)=\eta G(t)$ in $\mathbb{Q}\left[t, t^{-1}\right]$.

Proof We use the same argument with the proof of Lemma 2.15, but here we use the Chinese Remainder Theorem for the following ring isomorphism

$$
\mathbb{Q}\left[t, t^{-1}\right] /\left(t^{N}-1\right) \cong \bigoplus_{d \mid N, d \geq 1} \mathbb{Q}\left(\zeta_{d}\right)
$$

Note that $F(t)$ and $t^{N / 2} F(t)$ induce the control equivalent real value sequences by $u=t^{N / 2}$, but $\operatorname{red}\left(t^{N / 2} F(t)\right) \neq F(t)$ in general. Thus we have to care the case (ii) in Lemma 2.15] and 2.16. Here, we study relation between the coefficients of $F(t)$ and those of $\operatorname{red}\left(t^{N / 2} F(t)\right)$.

Lemma 2.17 Let $N$ be an even integer.

$$
\text { If } F(t)=a_{0}+\sum_{i=1}^{N / 2} a_{i}\left(t^{i}+t^{-i}\right), \text { then } \operatorname{red}\left(t^{N / 2} F(t)\right)=b_{0}+\sum_{i=1}^{N / 2} b_{i}\left(t^{i}+t^{-i}\right)
$$

with

$$
b_{0}=2 a_{N / 2}, b_{N / 2}=a_{0} / 2 \text { and } b_{j}=a_{N / 2-j} \quad(j=1,2, \ldots, N / 2-1) .
$$


Proof It is because

$$
t^{N / 2}\left(t^{j}+t^{-j}\right)=t^{N / 2+j}+t^{N / 2-j} \equiv t^{(N / 2-j)}+t^{-(N / 2-j)} \bmod t^{N}-1 .
$$

This will be used in the proof of Lemma 4.9, see also Remark 4.7,

\section{Alexander polynomial of $A_{m, n}$}

We compute the Alexander polynomial of the link $A_{m, n}$.

Definition 3.1 For a coprime positive pair $(m, n)$, we define a set $\mathfrak{I}(m, n)$ by

$$
\begin{aligned}
\Im(m, n) & :=(m \mathbb{Z} \cup n \mathbb{Z}) \cap\{k \in \mathbb{Z} \mid 0 \leq k \leq m n\} \\
& =\{0, m, 2 m, \ldots, n m\} \cup\{0, n, 2 n, \ldots, m n\} .
\end{aligned}
$$

Note that the cardinality of $\mathfrak{I}(m, n)$ is $m+n$. We sort the all elements in $\mathfrak{I}(m, n)$ as

$$
0=k_{0}<k_{1}<k_{2}<\cdots<k_{m+n-1}=m n \quad\left(k_{i} \in \mathfrak{I}(m, n)\right) .
$$

Here, $k_{1}$ is the smaller one in $m$ and $n$.

The goal of this section is:

Theorem 3.2 The Alexander polynomial of $A_{m, n}$ is

$$
\Delta_{A_{m, n}}(t, x) \doteq \sum_{i=0}^{m+n-1} t^{k_{i}} x^{i},
$$

where $t$ (and $x$, respectively) is represented by a meridian of $K_{1}$ (that of $K_{2}$ ).

Example 3.3 In the case $(m, n)=(3,5)$, we have $\mathfrak{I}(3,5)=\{0,3,5,6,9,10,12,15\}$ and

$$
\Delta_{A_{3,5}}(t, x) \doteq t^{15} x^{7}+t^{12} x^{6}+t^{10} x^{5}+t^{9} x^{4}+t^{6} x^{3}+t^{5} x^{2}+t^{3} x+1 \text {. }
$$

\subsection{Alexander matrix of $A_{m, n}$}

We start the proof of Theorem 3.2 with the following lemma:

Lemma 3.4 The Alexander matrix of $A_{m, n}$ is

$$
I_{m+n-1}-x M(m, n)
$$

and the Alexander polynomial of $A_{m, n}$ is obtained by

$$
\Delta_{A_{m, n}}(t, x) \doteq \operatorname{det}\left(I_{m+n-1}-x M(m, n)\right),
$$

where $M(m, n)$ is the $(m+n-1) \times(m+n-1)$-matrix of the form

$$
M(m, n):=\left[\begin{array}{ccc}
O_{n-1, m-1} & -\overrightarrow{T_{n-1}} & I_{n-1} \\
o_{m-1} & -t^{n} & o_{n-1} \\
t^{n} I_{m-1} & -t^{n} \overrightarrow{i_{m-1}} & O_{m-1, n-1}
\end{array}\right],
$$


$\overrightarrow{T_{n-1}}, \overrightarrow{i_{m-1}}$ are the following column vectors of size $(n-1) \times 1$ and $(m-1) \times 1$ respectively

$$
\overrightarrow{T_{n-1}}:=\left[\begin{array}{c}
t \\
t^{2} \\
\vdots \\
t^{n-1}
\end{array}\right], \quad \overrightarrow{i_{m-1}}:=\left[\begin{array}{c}
1 \\
1 \\
\vdots \\
1
\end{array}\right],
$$

$O_{s, s^{\prime}}$ (and $o_{s}$, respectively) is the zero matrix of size $s \times s^{\prime}$ (and of size $1 \times s$ ) and $I_{s}$ is the identity matrix of size $s \times s$.

Example 3.5

$M(3,5)=\left[\begin{array}{ccccccc}0 & 0 & -t & 1 & 0 & 0 & 0 \\ 0 & 0 & -t^{2} & 0 & 1 & 0 & 0 \\ 0 & 0 & -t^{3} & 0 & 0 & 1 & 0 \\ 0 & 0 & -t^{4} & 0 & 0 & 0 & 1 \\ 0 & 0 & -t^{5} & 0 & 0 & 0 & 0 \\ t^{5} & 0 & -t^{5} & 0 & 0 & 0 & 0 \\ 0 & t^{5} & -t^{5} & 0 & 0 & 0 & 0\end{array}\right], I-x M(3,5)=\left[\begin{array}{ccccccc}1 & 0 & t x & -x & 0 & 0 & 0 \\ 0 & 1 & t^{2} x & 0 & -x & 0 & 0 \\ 0 & 0 & 1+t^{3} x & 0 & 0 & -x & 0 \\ 0 & 0 & t^{4} x & 1 & 0 & 0 & -x \\ 0 & 0 & t^{5} x & 0 & 1 & 0 & 0 \\ -t^{5} x & 0 & t^{5} x & 0 & 0 & 1 & 0 \\ 0 & -t^{5} x & t^{5} x & 0 & 0 & 0 & 1\end{array}\right]$.

Proof of Lemma 3.4 We assumed that $2 \leq m<n$ (Section 1). We take the generators

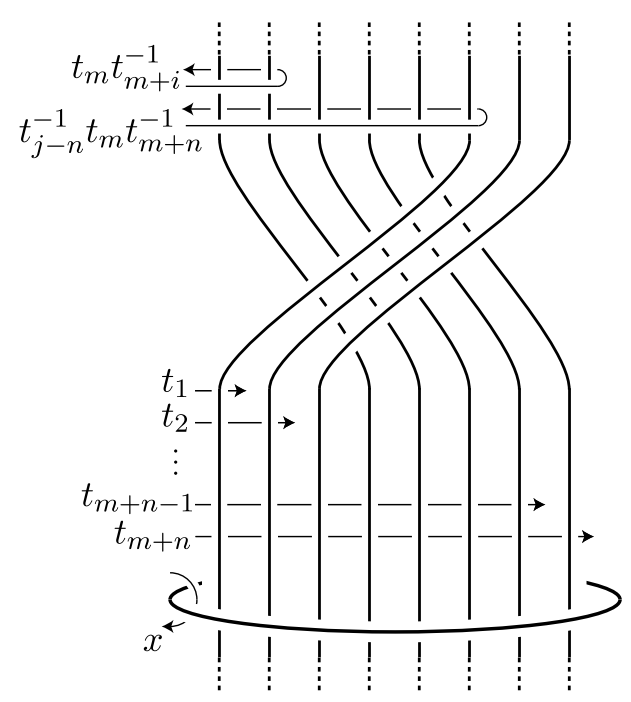

Figure 3: Generators $t_{i}, t_{j} \quad($ ex. $(m, n)=(3,5))$

of the fundamental group $\pi_{1}\left(S^{3} \backslash A_{m, n}\right)$ as in Figure 3. Then we have a presentation of the group:

$$
\begin{aligned}
& \left\langle\begin{array}{c|lr}
t_{1}, t_{2}, \ldots, t_{m+n} & \begin{array}{lr}
L_{i}:=t_{i} x t_{m} t_{m+i}^{-1} x^{-1} \\
R_{j}:=t_{j} x t_{j-n}^{-1} t_{m} t_{m+n}^{-1} x^{-1} & (j=n+1, n+2, \ldots, m+n)
\end{array}
\end{array}\right\} \\
& =\left\langle\begin{array}{c|lr}
t_{1}, t_{2}, \ldots, t_{m+n-1} & \begin{array}{lr}
L_{i}:=t_{i} x t_{m} t_{m+i}^{-1} x^{-1} & (i=1,2, \ldots, n-1) \\
C:=t_{n} x t_{m} x t_{m}^{-1} x^{-1} t_{n}^{-1} x^{-1} & \\
R_{j}:=t_{j} x t_{j-n}^{-1} x^{-1} t_{n}^{-1} & (j=n+1, \ldots, m+n-1)
\end{array}
\end{array}\right\},
\end{aligned}
$$


where we canceled $t_{m+n}=x^{-1} t_{n} x t_{m}$ by the relation $L_{n}$. Then $R_{m+n}$ is changed to the relation $C$, and the others $R_{j}$ are also changed. The non-zero free differentials of the relations $L_{i}$ and $R_{j}$ by $t_{*}$ are:

$$
\begin{aligned}
& \frac{\partial L_{i}}{\partial t_{i}}=1, \quad \frac{\partial L_{i}}{\partial t_{m}}=\delta_{i m}+t_{i} x, \quad \frac{\partial L_{i}}{\partial t_{m+i}}=-t_{i} x t_{m} t_{m+i}^{-1} \\
& \frac{\partial R_{j}}{\partial t_{j}}=1, \quad \frac{\partial R_{j}}{\partial t_{j-n}}=-t_{j} x t_{j-n}^{-1}, \quad \frac{\partial R_{j}}{\partial t_{n}}=-t_{j} x t_{j-n}^{-1} x^{-1} t_{n}^{-1},
\end{aligned}
$$

where $\delta_{i m}$ is Kronecker's delta. The non-zero free differentials of $C$ by $t_{*}$ are

$$
\frac{\partial C}{\partial t_{m}}=t_{n} x-t_{n} x t_{m} x t_{m}^{-1}, \quad \frac{\partial C}{\partial t_{n}}=1-t_{n} x t_{m} x t_{m}^{-1} x^{-1} t_{n}^{-1} .
$$

We let $\gamma$ denote the Hurewicz epimorphism $\gamma: \pi_{1}\left(S^{3} \backslash A_{m, n}\right) \rightarrow H_{1}\left(S^{3} \backslash A_{m, n} ; \mathbb{Z}\right) \cong\langle t, x \mid-\rangle$, defined as $\gamma\left(t_{i}\right)=t^{i}, \gamma(x)=x$. We redefine $L_{n}:=C$ and $L_{i}:=R_{i}$ for $n+1 \leq i \leq m+n-1$. Then the well-known formula ([Kaw, Lemma 7.3.2 (p.93)]) on the Alexander polynomial of links says

$$
\operatorname{det}\left[\gamma\left(\frac{\partial L_{i}}{\partial t_{j}}\right)\right] \doteq(1-x) \Delta_{A_{m, n}}(t, x),
$$

where $j$ (columns) runs in $1 \leq j \leq m+n-1$.

A submatrix from the first row to the $(n-1)$-th row of $\left[\gamma\left(\partial L_{i} / \partial t_{j}\right)\right]$ coincides with $I-x M(m, n)$ by the first half of (3.1). In the $n$-th row of $\left[\gamma\left(\partial L_{i} / \partial t_{j}\right)\right]$, non-zero entries are only

$$
\gamma\left(\frac{\partial C}{\partial t_{m}}\right)=(1-x) t^{n} x \text { at }(n, m) \text { and } \gamma\left(\frac{\partial C}{\partial t_{n}}\right)=(1-x) \text { at }(n, n),
$$

by (3.2). Thus the $n$-th row of $\left[\gamma\left(\partial L_{i} / \partial t_{j}\right)\right]$ coincides with $(1-x)$ times the $n$-th row of $I-x M(m, n)$. We add $1 /(1-x)$ times the $n$-th row of $\left[\gamma\left(\partial L_{i} / \partial t_{j}\right)\right]$ to each $j$-th row of $\left[\gamma\left(\partial L_{i} / \partial t_{j}\right)\right]$ with $j \geq n+1$. The resulting $j$-th row coincides with the $j$-th row of $I-x M(m, n)$ by the second half of (3.1). Therefore we have

$$
\operatorname{det}\left[\gamma\left(\frac{\partial L_{i}}{\partial t_{j}}\right)\right] \doteq(1-x) \cdot \operatorname{det}(I-x M(m, n)) .
$$

Remark 3.6 The matrix $M(m, n)$ can also be obtained by the Burau representation (see Birman [Bir, p.121]) of the braid group.

\subsection{Properties of $\mathfrak{I}(m, n)$}

We need some properties on elements $k_{i}$ of $\mathfrak{I}(m, n)$, see Definition 3.1. They will be also used in Subsection 4.2, Subsection 5.4.

Definition 3.7 We define $u_{j}(j=0,1,2, \ldots, n)$ and $w_{j}(j=0,1,2, \ldots, m)$ by

$$
k_{u_{j}}=j m \quad \text { and } \quad k_{w_{j}}=j n .
$$


It is easy to see:

\section{Proposition 3.8}

(1) $k_{i}+k_{m+n-1-i}=m n, \quad(i=0,1, \ldots, m+n-1)$.

(2) $u_{0}=w_{0}=0, u_{n}=w_{m}=m+n-1$, and

$$
u_{j}=\left[\frac{j m}{n}\right]+j \quad(j=1,2, \ldots, n-1), \quad w_{j}=\left[\frac{j n}{m}\right]+j \quad(j=1,2, \ldots, m-1),
$$

where $[\cdot]$ is the gaussian symbol.

(3) Both $u_{j}, w_{j}$ are increasing sequences.

(4) $u_{j}+u_{n-j}=w_{j}+w_{m-j}=m+n-1$.

$$
\sum_{i=0}^{m+n-1} t^{k_{i}} x^{i}=1+\sum_{i=1}^{n-1} t^{i m} x^{u_{i}}+\sum_{j=1}^{m-1} t^{j n} x^{w_{j}}+t^{m n} x^{m+n-1}
$$

Notation 3.9 For an integer $N$, we denote by $[N]_{n}$ the unique integer satisfying

$$
[N]_{n} \equiv N \quad(\bmod n) \quad \text { and } \quad 0 \leq[N]_{n} \leq n-1
$$

We let $\sigma$ (and its inverse $\sigma^{\prime}$, respectively) denote the bijection

$$
\sigma, \sigma^{\prime}:\{0,1,2, \ldots, n-1\} \rightarrow\{0,1,2, \ldots, n-1\}
$$

defined by $\sigma(i):=[i \bar{m}]_{n}$ (and $\left.\sigma^{\prime}(i):=[i m]_{n}\right)$, where $\bar{m}$ is regarded as an integral lift of $\bar{m}(\bmod n)$. We also define

$$
\rho(i):=\frac{\sigma(i) m-i}{n} \quad(i=1,2, \ldots, n-1) .
$$

By the definition of $\sigma(i), \rho(i)$ is an integer.

\section{Lemma 3.10}

(1) $\sigma(0)=0, \sigma(m)=1$ and $\sigma(n-m)=n-1$.

(2) For $i=1,2, \ldots, n-1$ and $i \neq n-m$, we have $u_{\sigma(i)+1}-u_{\sigma(i)}=1$ or 2 . Furthermore, $u_{\sigma(i)+1}-u_{\sigma(i)}=2$ if and only if $n-m+1 \leq i \leq n-1$.

(3) $\sigma(m+i)=\sigma(i)+1, u_{\sigma(m+i)}=u_{\sigma(i)}+1, \quad(i=1,2, \ldots, n-m-1)$.

(4) $\sigma(n-m+i)=\sigma(i)-1, u_{\sigma(n-m+i)}=u_{\sigma(i)}-2, \quad(i=1,2, \ldots, m-1)$.

(5) $\rho:\{1,2, \ldots, m-1\} \rightarrow\{1,2, \ldots, m-1\}$ is a bijection.

(6) $w_{\rho(i)}=u_{\sigma(i)}-1,(i=1,2, \ldots, m-1)$. 
Proof (1) It is easy to see.

(2) Suppose that $i=1,2, \ldots, n-1$ and $i \neq n-m$. Since $\sigma$ is a bijection, (1) and Proposition 3.8 (3), we have $1 \leq \sigma(i) \leq n-2$ and $u_{\sigma(i)+1}-u_{\sigma(i)}=1$ or 2 . We set $j=\sigma(i)$. It holds that $u_{j+1}-u_{j}=2$ if and only if there exists an integer $j^{\prime}$ such that $j m<j^{\prime} n<(j+1) m$, which implies $j^{\prime} n-m<j m<j^{\prime} n$ and

$$
n-m<[j m]_{n}<n .
$$

Since $[j m]_{n}=\sigma^{\prime}(j)=\sigma^{\prime} \circ \sigma(i)=i$, we have $n-m+1 \leq i \leq n-1$.

(3) Suppose that $i=1,2, \ldots, n-m-1$. We have $1 \leq \sigma(i) \leq n-2$ again. Since $\sigma(m+i) \equiv$ $(m+i) \bar{m} \equiv \sigma(i)+1(\bmod n)$, we have $\sigma(m+i)=\sigma(i)+1$. By $(2)$, we have $u_{\sigma(m+i)}=$ $u_{\sigma(i)+1}=u_{\sigma(i)}+1$.

(4) Suppose that $i=1,2, \ldots, m-1$. We have $2 \leq \sigma(i) \leq n-1$. Since $\sigma(n-m+i) \equiv(n-m+$ $i) \bar{m} \equiv \sigma(i)-1(\bmod n)$, we have $\sigma(n-m+i)=\sigma(i)-1$. By $(2)$ and $n-m+1 \leq n-m+i \leq n-1$, we have $u_{\sigma(n-m+i)}=u_{\sigma(i)}-2$.

(5) Suppose that $i=1,2, \ldots, m-1$. Since $\sigma(i) m=[i \bar{m}]_{n} m \equiv i(\bmod n)$, and $0 \leq \sigma(i) \leq n-1$, we have

$$
0 \leq \rho(i)=\frac{\sigma(i) m-i}{n} \leq \frac{(n-1) m}{n}<m,
$$

and hence the image of the map $\rho$ is included in $\{0,1,2, \ldots, m-1\}$. By the definition, we have $\rho(i) n \equiv-i(\bmod m)$, thus $\rho$ is bijective. In fact, $\rho(i)=[(m-i) \bar{n}]_{m}$.

(6) Suppose that $i=1,2, \ldots, m-1$. By (4), we have $u_{\sigma(n-m+i)}=u_{\sigma(i)-1}=u_{\sigma(i)}-2$. Then, as in the proof of $(2)$, there exists an integer $j$ such that $w_{j}=u_{\sigma(i)}-1$ such that

$$
(\sigma(i)-1) m=k_{u_{\sigma(i)-1}}<k_{w_{j}}=j n<k_{u_{\sigma(i)}}=\sigma(i) m .
$$

Since $\sigma(i) m-i \equiv 0(\bmod n)$, we have $j n=\sigma(i) m-i$ and $j=\rho(i)$.

Definition 3.11 We define the monomial $e_{i}$ of $t, x$ by

$$
e_{i}=t^{\sigma(i) m-i} x^{u_{\sigma(i)}}, \quad(i=1,2, \ldots, n-1) .
$$

\section{Lemma 3.12}

(1) $e_{m}=x$ and $e_{n-m}=t^{m n-n} x^{m+n-2}$.

(2) $e_{m+i}=x e_{i},(i=1,2, \ldots, n-m-1)$.

(3) $e_{n-m+i}=t^{-n} x^{-2} e_{i},(i=1,2, \ldots, m-1)$.

(4) $t^{\sigma(i) m-i} x^{u_{\sigma(i)}-1}=t^{\rho(i) n} x^{w_{\rho(i)}},(i=1,2, \ldots, m-1)$.

Proof They are proved by the Lemma $3.10(1),(3),(4)$ and (6) respectively. 


\subsection{Proof of Theorem 3.2 (Alexander polynomial of $A_{m, n}$ )}

We regard

$$
\sum_{i=0}^{m+n-1} t^{k_{i}} x^{i}=0
$$

as an algebraic equation over $\mathbb{Z}\left[t, t^{-1}\right]$, and $x$ as a root of the equation.

Lemma 3.13 The algebraic equation (3.3) over $\mathbb{Z}\left[t, t^{-1}\right]$ has no multiple root.

Proof If we substitute $t=1$ into the equation (3.3), then we have

$$
\sum_{i=0}^{m+n-1} x^{i}=0 .
$$

Since this equation has no multiple root, and its degree is equal to that of (3.3) $(=m+n-1)$, the equation (3.3) has no multiple root.

Let $\boldsymbol{v}$ be a row vector of size $1 \times(m+n-1)$, decomposed as

$$
\boldsymbol{v}=\left[\begin{array}{lll}
\boldsymbol{e} & f & \boldsymbol{g}
\end{array}\right]
$$

where $f$ is a scalar and

$$
\boldsymbol{e}=\left[\begin{array}{llll}
e_{1} & e_{2} & \cdots & e_{n-1}
\end{array}\right], \quad \boldsymbol{g}=\left[\begin{array}{llll}
g_{1} & g_{2} & \cdots & g_{m-1}
\end{array}\right]
$$

are row vectors of size $1 \times(n-1)$ and $1 \times(m-1)$, respectively. Then the matrix $M(m, n)$ in Lemma 3.4 satisfies

$$
\boldsymbol{v} \cdot M(m, n)=\left[\begin{array}{lll}
t^{n} \boldsymbol{g} & \left(-\boldsymbol{e} \cdot \overrightarrow{T_{n-1}}-t^{n} f-t^{n} \boldsymbol{g} \cdot \overrightarrow{i_{m-1}}\right) & \boldsymbol{e}
\end{array}\right] .
$$

Lemma 3.14 If we take $e_{i}=t^{\sigma(i) m-i} x^{u_{\sigma(i)}}(i=1,2, \ldots, n-1)$ as in Definition [3.11, $f=x e_{n-m}$ and $g_{i}=t^{-n} x^{-1} e_{i}(i=1,2, \ldots, m-1)$, then we have

$$
\boldsymbol{v} \cdot M(m, n)=x^{-1} \boldsymbol{v} .
$$

Proof By Lemma 3.12, and the definition of $e_{i}$ and $g_{i}$, we can see immediately that the $i$-th entry of $\boldsymbol{v} \cdot M(m, n)$ is equal to that of $M(m, n)$ multiplied by $x^{-1}$ except the case $i=m$. The $m$-th entry of (3.4) is computed as

$$
\begin{aligned}
-\boldsymbol{e} \cdot \overrightarrow{T_{n-1}}-t^{n} f-t^{n} \boldsymbol{g} \cdot \overrightarrow{i_{m-1}} & =-\sum_{i=1}^{n-1} t^{i} e_{i}-t^{n} f-t^{n} \sum_{j=1}^{m-1} g_{j} \\
& =-\sum_{i=1}^{n-1} t^{\sigma(i) m} x^{u_{\sigma(i)}}-t^{m n} x^{m+n-1}-\sum_{j=1}^{m-1} t^{\rho(j) n} x^{w_{\rho(j)}} \\
& =1
\end{aligned}
$$


by Definition 3.11, Lemma 3.12, bijectivities of $\sigma$ and $\rho$, the equation (3.3) and Proposition 3.8 (5). We have the lemma.

Proof of Theorem 3.2 By Lemma 3.14, $x^{-1}$ is an eigenvalue of the matrix $M(m, n)$ where $x$ is a root of (3.3). Since the degree of the equation (3.3) and the size of $M(m, n)$ are identical $(=m+n-1)$, and the equation (3.3) have no multiple root by Lemma 3.13, we have

$$
\sum_{i=0}^{m+n-1} t^{k_{i}} x^{i}=x^{m+n-1} \cdot \operatorname{det}\left(x^{-1} I_{m+n-1}-M(m, n)\right) .
$$

By Lemma 3.4, we have the result.

\section{Reidemeister torsions of $\left(A_{m, n} ; r, 0\right)$ and key lemmas}

We compute the Reidemeister torsions of $\left(A_{m, n} ; r, 0\right)$. The goal is Lemma 4.3.

\subsection{The first homology of $\left(A_{m, n} ; r, 0\right)$}

We calculate the first homology of $M=\left(A_{m, n} ; \alpha / \beta, 0\right)$. Let $E$ denote the complement of $A_{m, n}$. We regard $M=E \cup V_{1} \cup V_{2}$, see Notation in Subsection 2.1 We set $M_{1}:=E \cup V_{1} \subset M$.

From now on, we always assume that $\operatorname{gcd}(\alpha, \beta)=1, \beta>0$, and

$$
\operatorname{gcd}(m+n, \alpha)=1
$$

which is equivalent to the condition for the first homology $H_{1}(M ; \mathbb{Z})$ to be finite cyclic by the elementary divisor theory. Then the order is $(m+n)^{2} \beta: H_{1}(M ; \mathbb{Z}) \cong \mathbb{Z} /(m+n)^{2} \beta \mathbb{Z}$.

We determine the first homologies of $E, M_{1}$ and $M$, define generators and study relations. First, $H_{1}(E)$ is a free abelian group of rank 2 generated by $\left[m_{1}\right]$ and $\left[m_{2}\right]$ :

$$
H_{1}(E) \cong\left\langle\left[m_{1}\right],\left[m_{2}\right] \mid-\right\rangle \cong \mathbb{Z}^{2} .
$$

We have

$$
\left[l_{1}\right]=\left[m_{2}\right]^{m+n} \quad \text { and } \quad\left[l_{2}\right]=\left[m_{1}\right]^{m+n} .
$$

Next, we attach $V_{1}$ to $E$ to make $M_{1}$. We take integers $\gamma, \delta$ such that $\alpha \delta-\beta \gamma=-1$, and fix the meridian-longitude system $m_{1}^{\prime}, l_{1}^{\prime}$ of the solid torus $V_{1}$. In $H_{1}\left(M_{1}\right)$, we have the relations (4.1) and

$$
\left[m_{1}^{\prime}\right]=\left[m_{1}\right]^{\alpha}\left[l_{1}\right]^{\beta}=1 \quad \text { and } \quad\left[l_{1}^{\prime}\right]=\left[m_{1}\right]^{\gamma}\left[l_{1}\right]^{\delta} .
$$

Thus, we have

$$
\begin{aligned}
H_{1}\left(M_{1}\right) & \cong\left\langle\left[m_{1}\right],\left[m_{2}\right] \mid\left[m_{1}\right]^{\alpha}\left[m_{2}\right]^{(m+n) \beta}=1\right\rangle \\
& \cong\langle T \mid-\rangle \cong \mathbb{Z}
\end{aligned}
$$


where $T=\left[m_{1}\right]^{\gamma^{\prime}}\left[m_{2}\right]^{\delta^{\prime}}$ by taking integers $\gamma^{\prime}, \delta^{\prime}$ satisfying $\alpha \delta^{\prime}-p \beta \gamma^{\prime}=-1$. By the relations above, we have

$$
\begin{aligned}
{\left[m_{1}\right] } & =\left[m_{1}\right]^{-\alpha \delta^{\prime}+(m+n) \beta \gamma^{\prime}} \\
& =\left(\left[m_{1}\right]^{\alpha}\left[m_{2}\right]^{(m+n) \beta}\right)^{-\delta^{\prime}}\left(\left[m_{1}\right]^{\gamma^{\prime}}\left[m_{2}\right]^{\delta^{\prime}}\right)^{(m+n) \beta}=T^{(m+n) \beta}, \\
{\left[m_{2}\right] } & =\left[m_{2}\right]^{-\alpha \delta^{\prime}+(m+n) \beta \gamma^{\prime}} \\
& =\left(\left[m_{1}\right]^{\alpha}\left[m_{2}\right]^{(m+n) \beta}\right)^{\gamma^{\prime}}\left(\left[m_{1}\right]^{\gamma^{\prime}}\left[m_{2}\right]^{\delta^{\prime}}\right)^{-\alpha}=T^{-\alpha}, \\
{\left[l_{1}^{\prime}\right] } & =\left[m_{1}\right]^{\gamma}\left[l_{1}\right]^{\delta} \\
& =\left[m_{1}\right]^{\gamma}\left[m_{2}\right]^{(m+n) \delta}=T^{(m+n) \beta \gamma-(m+n) \alpha \delta}=T^{m+n} .
\end{aligned}
$$

Finally, we attach $V_{2}$ to $M_{1}$ to make $M$. By (4.1) and (4.2) in $H_{1}(M)$, we have

$$
\left[m_{2}^{\prime}\right]=\left[l_{2}\right]=\left[m_{1}\right]^{m+n}=T^{(m+n)^{2} \beta}=1, \quad\left[l_{2}^{\prime}\right]=\left[m_{2}\right]=T^{-\alpha},
$$

and

$$
H_{1}(M) \cong\left\langle T \mid T^{(m+n)^{2} \beta}=1\right\rangle \cong \mathbb{Z} /(m+n)^{2} \beta \mathbb{Z} .
$$

\subsection{Reidemeister torsion of $\left(A_{m, n} ; r, 0\right)$}

In this subsection, we compute the Reidemeister torsion of $\left(A_{m, n} ; r, 0\right)$. The goal is Lemma 4.3 .

First, by Surgery formula II (Lemma 2.5) and the results on the first homology in the last subsection, we have

$$
\tau\left(M_{1}\right) \doteq \Delta_{A_{m, n}}\left(T^{(m+n) \beta}, T^{-\alpha}\right)\left(T^{m+n}-1\right)^{-1}
$$

By the Alexander polynomial in Theorem 3.2 and (4.3),

$$
\begin{aligned}
\tau\left(M_{1}\right) & \doteq\left(\sum_{i=0}^{m+n-1} T^{k_{i}(m+n) \beta-i \alpha}\right)\left(T^{m+n}-1\right)^{-1} \\
& =\frac{\sum_{i=0}^{m+n-1}\left(T^{k_{i}(m+n) \beta-i \alpha}-T^{-i \alpha}\right)}{\sum_{i=0}^{m+n-1} T^{-i \alpha}} \\
& =\sum_{i=0}^{m+n-1}\left(T^{-i \alpha} \cdot \frac{T^{k_{i}(m+n) \beta}-1}{T^{m+n}-1}\right)+\frac{T^{-(m+n) \alpha}-1}{T^{m+n}-1} \cdot\left(T^{-\alpha}-1\right)^{-1} .
\end{aligned}
$$

Note that $\left(T^{a b}-1\right) /\left(T^{b}-1\right)$ is a polynomial $1+T^{b}+T^{2 b}+\cdots+T^{(a-1) b}$.

Next, let $d \geq 2$ be a divisor of $(m+n)^{2} \beta$. It holds that $\operatorname{gcd}(d, \alpha)=1$. By Surgery formula I (Lemma 2.3) and the results on the first homology, the Reidemeister-Turaev torsion of $M$ is

$$
\tau^{\psi_{d}}(M) \doteq \tau^{\rho_{d}}\left(M_{1}\right)\left(\zeta_{d}^{\alpha}-1\right)^{-1}
$$

where $\rho_{d}:=\psi_{d} \circ \iota$ is the composite of a ring homomorphism $\iota: \mathbb{Z}\left[H_{1}\left(M_{1}\right)\right] \rightarrow \mathbb{Z}\left[H_{1}(M)\right]$ induced from the natural inclusion, and a ring homomorphism $\psi_{d}: \mathbb{Z}\left[H_{1}(M)\right] \rightarrow \mathbb{Q}\left(\zeta_{d}\right)$ such that $\psi_{d}(T)=\zeta_{d}$. 
We take $d$ as a divisor of $(m+n)$ and a ring homomorphism $\psi_{d}^{\prime}: \mathbb{Z}\left[H_{1}(M)\right] \rightarrow \mathbb{Q}\left(\zeta_{d}\right)$ such that $\psi_{d}^{\prime}(T)=\zeta_{d}^{-\bar{\alpha}}$ where $\alpha \bar{\alpha} \equiv 1(\bmod m+n)$. Then $d$ is still a divisor of $(m+n)^{2} \beta$, and $\zeta_{d}^{-\bar{\alpha}}$ is still a primitive $d$-th root of unity, since $\operatorname{gcd}(d, \alpha)=1$. By (4.4) and (4.5), we have

$$
\tau_{d}^{\psi_{d}^{\prime}}(M) \doteq\left\{\beta\left(\zeta_{d}-1\right) \sum_{i=0}^{m+n-1} k_{i} \zeta_{d}^{i}-\alpha\right\}\left(\zeta_{d}-1\right)^{-2} .
$$

Definition 4.1 For a divisor $d \geq 2$ of $(m+n)$, and the primitive $d$-th root $\left(\zeta=\zeta_{d}\right)$ of unity, we define

$$
R(m, n):=(\zeta-1) \sum_{i=0}^{m+n-1} k_{i} \zeta^{i}
$$

By (4.6), the Reidemeister-Turaev torsion of $M$ is expressed as

$$
\tau^{\psi_{d}^{\prime}}(M) \doteq\{\beta R(m, n)-\alpha\}(\zeta-1)^{-2} .
$$

\section{Lemma 4.2}

(1) $R(m, n)$ is a real number.

(2) $R(m, n)=m n+\frac{1}{2} \sum_{i=1}^{m+n-1}\left(k_{i-1}-k_{i}\right)\left(\zeta^{i}+\zeta^{-i}\right)$.

(3) $R(m, n)=m(n+1)+\sum_{j=1}^{m-1}\left(m-s_{j}\right)\left(\zeta^{w_{j}}+\zeta^{-w_{j}}\right)$,

where $s_{j}$ is defined by $s_{j}:=[j n]_{m}$ for an integer $j$, see Notation in Subsection 3.2 .

(4) $R(m, n)=m(n+1)+\sum_{j=1}^{m-1}(m-j)\left(\xi^{j}+\xi^{-j}\right)$.

(5) $R(m, n)=\xi^{-(m-1)} \cdot\left(\frac{\xi^{m}-1}{\xi-1}\right)^{2}+m n=\left|\frac{\xi^{m}-1}{\xi-1}\right|^{2}+m n$,

where $\xi=\zeta^{\bar{m}}$ with $m \bar{m} \equiv 1 \bmod (m+n)$.

Proof (1) The complex conjugate $\overline{R(m, n)}$ of $R(m, n)$ is

$$
\begin{aligned}
\overline{R(m, n)} & =\left(\zeta^{-1}-1\right) \sum_{i=0}^{m+n-1} k_{i} \zeta^{-i}=(\zeta-1) \sum_{i=0}^{m+n-1}-k_{i} \zeta^{-i-1} \\
& =(\zeta-1) \sum_{i=0}^{m+n-1}\left(m n-k_{i}\right) \zeta^{m+n-i-1} \\
& =(\zeta-1) \sum_{i=0}^{m+n-1} k_{m+n-i-1} \zeta^{m+n-i-1} \\
& =(\zeta-1) \sum_{i=0}^{m+n-1} k_{i} \zeta^{i}=R(m, n)
\end{aligned}
$$


by Proposition 3.8 (1) and the equality $1+\zeta+\cdots+\zeta^{m+n-1}=0$.

(2) First, we have

$$
\begin{aligned}
R(m, n) & =\sum_{i=0}^{m+n-1} k_{i}\left(\zeta^{i+1}-\zeta^{i}\right)=\sum_{i=1}^{m+n} k_{i-1} \zeta^{i}-\sum_{i=0}^{m+n-1} k_{i} \zeta^{i} \\
& =m n+\sum_{i=1}^{m+n-1}\left(k_{i-1}-k_{i}\right) \zeta^{i} .
\end{aligned}
$$

By the proof of (1), we have

$$
\begin{aligned}
R(m, n) & =\frac{1}{2}\{R(m, n)+\overline{R(m, n)}\} \\
& =m n+\frac{1}{2} \sum_{i=1}^{m+n-1}\left(k_{i-1}-k_{i}\right)\left(\zeta^{i}+\zeta^{-i}\right) .
\end{aligned}
$$

Before the proof of (3), (4) and (5), we prove the following:

Claim (Property on $s_{j}$ )

(a) The map from $j$ to $s_{j}\left(=[j n]_{m}\right)$ is a bijection on $\{1,2, \ldots, m-1\}$ to itself.

(b) It holds that $s_{j}+s_{m-j}=m$.

(c) There exists a unique element $h$ in $\{1,2, \ldots, m-1\}$ such that $\operatorname{gcd}(h, m)=1$ and $s_{h}=1$. It holds that $w_{h} \equiv-\bar{m}(\bmod m+n)$.

(d) For the same $h$ in (c) and each element $a$ in $\{1,2, \ldots, m-1\}$, we have

$$
s_{a h}=a \quad \text { and } \quad w_{a h} \equiv a w_{h} \quad(\bmod m+n),
$$

where we regard $w_{a h}$ as $w_{j}$ with $j=[a h]_{m}$, precisely.

Proof of Claim (a) The map is induced by the multiplication of $n$ (i.e., $j \mapsto j n$ ) over $(\mathbb{Z} / m \mathbb{Z}) \backslash\{0\}$. It is a bijection, since $\operatorname{gcd}(m, n)=1$.

(b) It is easy to see.

(c) By (a), there exists a unique element $h$ in $\{1,2, \ldots, m-1\}$ such that $s_{h}=1$. In fact, it holds that $h \equiv \bar{n}(\bmod m)$. We have $\operatorname{gcd}(h, m)=1$. The second half is shown by $-m w_{h}=$ $-m\left(h+\left[\frac{h n}{m}\right]\right) \equiv h n-m\left[\frac{h n}{m}\right]=[h n]_{m}=s_{h}=1 \quad(\bmod m+n)$, see Proposition $3.8(2)$.

(d) Since $s_{a h} \equiv a h n \equiv a(\bmod m)$, we have $s_{a h}=a$, for $a$ in $\{1,2, \ldots, m-1\}$. Since $m w_{a h}=m a h+m\left[\frac{a h n}{m}\right] \equiv-\left(a h n-m\left[\frac{a h n}{m}\right]\right)=-[a h n]_{m}=-s_{a h}=-a \quad(\bmod m+n)$.

(3) We go back to the expression (2). We divide the set $\{1,2, \ldots, m+n-1\}$ of indices of $k_{i}$, into $M, R$ and $L$ according to whether $k_{i-1}$ and/or $k_{i}$ belongs to $m \mathbb{Z} \cap \mathfrak{I}(m, n)$.

$$
\{1,2, \ldots, m+n-1\}=M \cup R \cup L \quad \text { (a disjoint union) }
$$




\begin{tabular}{llc} 
Definition of the subset & parameter $j$ & $k_{i-1}-k_{i}$ \\
\hline$M:=\left\{i \mid k_{i-1} \in m \mathbb{Z}\right.$ and $\left.k_{i} \in m \mathbb{Z}\right\}$ & - & $-m$ \\
$R:=\left\{i \mid k_{i-1} \in m \mathbb{Z}\right.$ and $\left.k_{i} \notin m \mathbb{Z}\right\}$ & $k_{i}=j n$ & $m\left[\frac{j n}{m}\right]-j n$ \\
$L:=\left\{i \mid k_{i-1} \notin m \mathbb{Z}\right.$ and $\left.k_{i} \in m \mathbb{Z}\right\}$ & $k_{i-1}=j n$ & $j n-m\left(\left[\frac{j n}{m}\right]+1\right)$ \\
\hline
\end{tabular}

Note that the case both $k_{i-1} \notin m \mathbb{Z}$ and $k_{i} \notin m \mathbb{Z}$ (in other words, the case that both $k_{i-1}$ and $k_{i}$ belong to $n \mathbb{Z}$ ) never occur, since $m<n$ (Section 1).

For each $i \in R$, there exists $j$ with $1 \leq j<m$ such that $k_{i}=j n$, equivalently $i=w_{j}$. Then, by Proposition $3.8(4), i^{\prime}:=w_{m-j}+1$ belongs to $L$ and it holds that $i^{\prime}=\left(m+n-1-w_{j}\right)+1=$ $m+n-i$. The correspondence between $i \in R$ and $i^{\prime} \in L$ above is one to one. It also holds that $\zeta^{i^{\prime}}=\zeta^{w_{m-j}+1}=\zeta^{m+n-i}=\zeta^{-i}$ and

$$
\begin{aligned}
k_{i-1}-k_{i} & =m\left[\frac{j n}{m}\right]-j n=-[j n]_{m}=-s_{j}, \\
k_{i^{\prime}-1}-k_{i^{\prime}} & =(m-j) n-m\left(\left[\frac{(m-j) n}{m}\right]+1\right) \\
& =[(m-j) n]_{m}-m=s_{m-j}-m=-s_{j},
\end{aligned}
$$

by Claim (b). Thus

$$
\begin{aligned}
& \left(k_{i-1}-k_{i}\right)\left(\zeta^{i}+\zeta^{-i}\right)+\left(k_{i^{\prime}-1}-k_{i^{\prime}}\right)\left(\zeta^{i^{\prime}}+\zeta^{-i^{\prime}}\right) \\
& =-2 s_{j}\left(\zeta^{i}+\zeta^{-i}\right) \\
& =-2 s_{j}\left(\zeta^{w_{j}}+\zeta^{-w_{j}}\right)
\end{aligned}
$$

and

$$
\begin{aligned}
& \sum_{i \in R}\left(k_{i-1}-k_{i}+m\right)\left(\zeta^{i}+\zeta^{-i}\right)+\sum_{i \in L}\left(k_{i-1}-k_{i}+m\right)\left(\zeta^{i}+\zeta^{-i}\right) \\
& =\sum_{i \in R}\left\{\left(k_{i-1}-k_{i}+m\right)\left(\zeta^{i}+\zeta^{-i}\right)+\left(k_{i^{\prime}-1}-k_{i^{\prime}}+m\right)\left(\zeta^{i^{\prime}}+\zeta^{-i^{\prime}}\right)\right\} \\
& =2 \sum_{j=1}^{m-1}\left(m-s_{j}\right)\left(\zeta^{w_{j}}+\zeta^{-w_{j}}\right) .
\end{aligned}
$$


Thus, using $1+\zeta+\zeta^{2}+\cdots+\zeta^{m+n-1}=0$,

$$
\begin{aligned}
R(m, n) & =m n+\frac{1}{2} \sum_{i=1}^{m+n-1}\left(k_{i-1}-k_{i}\right)\left(\zeta^{i}+\zeta^{-i}\right) \\
& =m n+\frac{1}{2} \sum_{i=1}^{m+n-1}\left(k_{i-1}-k_{i}\right)\left(\zeta^{i}+\zeta^{-i}\right)+\frac{1}{2} \sum_{i=0}^{m+n-1} m\left(\zeta^{i}+\zeta^{-i}\right) \\
& =m n+m+\frac{1}{2} \sum_{i=1}^{m+n-1}\left(k_{i-1}-k_{i}+m\right)\left(\zeta^{i}+\zeta^{-i}\right) \\
& =m(n+1)+\frac{1}{2} \sum_{i \in M \cup R \cup L}\left(k_{i-1}-k_{i}+m\right)\left(\zeta^{i}+\zeta^{-i}\right) \\
& =m(n+1)+\sum_{j=1}^{m-1}\left(m-s_{j}\right)\left(\zeta^{w_{j}}+\zeta^{-w_{j}}\right) .
\end{aligned}
$$

(4) We take $h(=\bar{n} \bmod m)$ in Claim (c). By Claim (d), we have

$$
\begin{aligned}
R(m, n) & =m(n+1)+\sum_{j=1}^{m-1}\left(m-s_{j}\right)\left(\zeta^{w_{j}}+\zeta^{-w_{j}}\right) \\
& =m(n+1)+\sum_{a=1}^{m-1}\left(m-s_{a h}\right)\left(\zeta^{w_{a h}}+\zeta^{-w_{a h}}\right) \\
& =m(n+1)+\sum_{a=1}^{m-1}(m-a)\left(\zeta^{-a \bar{m}}+\zeta^{a \bar{m}}\right) \\
& =m(n+1)+\sum_{a=1}^{m-1}(m-a)\left(\xi^{a}+\xi^{-a}\right),
\end{aligned}
$$

where we set $\xi=\zeta^{\bar{m}}$, which is also a $d$-th primitive root of unity.

(5) Using elementary calculus

$$
\sum_{a=1}^{m-1}(m-a) x^{a}=\frac{x^{m}-m x-1}{x-1}+\frac{x^{m}-1}{(x-1)^{2}}=x \frac{x^{m}-1}{(x-1)^{2}}-m \frac{x}{x-1},
$$

we have

$$
R(m, n)=\xi^{-(m-1)} \cdot\left(\frac{\xi^{m}-1}{\xi-1}\right)^{2}+m n .
$$

The proof of Lemma 4.2 is completed.

The result of this subsection is summarized as:

Lemma 4.3 Let $M=\left(A_{m, n} ; \alpha / \beta, 0\right)$, and $d \geq 2$ a divisor of $m+n$. Then the ReidemeisterTuraev torsion of $M$ related with $\psi_{d}^{\prime}(T)=\zeta_{d}^{-\bar{\alpha}}$ is

$$
\begin{aligned}
\tau^{\psi_{d}^{\prime}}(M) & \doteq \xi^{-m}\{\beta R(m, n)-\alpha\}\left(\xi^{m}-1\right)^{-2} \\
& =\xi^{-m}\left\{\beta\left|\frac{\xi^{m}-1}{\xi-1}\right|^{2}-(\alpha-m n \beta)\right\}\left(\xi^{m}-1\right)^{-2}
\end{aligned}
$$


where $\xi=\zeta^{\bar{m}}$ (thus $\xi^{m}=\zeta$ ) is a primitive $d$-th root of unity.

\subsection{Necessary conditions}

We study some necessary conditions for $\left(A_{m, n} ; \alpha / \beta, 0\right)$ to be a lens space by the ReidemeisterTuraev torsions.

Lemma 4.4 Suppose that $\left(A_{m, n} ; \alpha / \beta, 0\right)$ is a lens space. Then there exists integers $i$ and $j$ such that $\operatorname{gcd}(i, m+n)=\operatorname{gcd}(j, m+n)=1$ and

$$
\{\beta R(m, n)-\alpha\}\left(\xi^{m}-1\right)^{-2} \doteq\left(\xi^{i}-1\right)^{-1}\left(\xi^{j}-1\right)^{-1},
$$

equivalently,

$$
\left\{\beta\left|\frac{\xi^{m}-1}{\xi-1}\right|^{2}-\alpha^{\prime}\right\}\left(\xi^{m}-1\right)^{-2} \doteq \frac{1}{\left(\xi^{i}-1\right)\left(\xi^{j}-1\right)},
$$

where $\alpha^{\prime}=\alpha-m n \beta$, and $\xi$ is a primitive $d$-th root of unity.

The equalities (4.8), (4.9) correspond to two expressions of $\tau^{\psi_{d}^{\prime}}(M)$ in Lemma 4.3.

Lemma 4.5 Let $d \geq 2$ be a divisor of $m+n$. Suppose that $\left(A_{m, n} ; \alpha / \beta, 0\right)$ is a lens space. Then we have

(1) $\left|N_{d}(\beta R(m, n)-\alpha)\right|=1$, where $N_{d}$ is the $d$-norm, see Subsection 2.2.

(2) $\alpha^{\prime}=\alpha-m n \beta \geq 0$.

Proof (1) We take the $d$-norm of the equality (4.8). Since $N_{d}\left(\xi^{m}-1\right)=N_{d}\left(\xi^{i}-1\right)=$ $N_{d}\left(\xi^{j}-1\right) \neq 0$ by Proposition 2.8 (1) and Lemma 2.9, we have the result.

(2) Suppose that the integer $\alpha^{\prime}=\alpha-m n \beta<0$. Then we have

$$
\beta R(m, n)-\alpha=\beta\left|\frac{\xi^{m}-1}{\xi-1}\right|^{2}-\alpha^{\prime}>1
$$

hence $\left|N_{d}(\beta R(m, n)-\alpha)\right|>1$. By (1), we have the result.

Fixing the combinatorial Euler structure, we will regard (4.9) as a control equivalence of the value sequences of degree $m+n$, in the sense of Lemma 2.12 (1). Note that the first factor in the left-hand side is a real value. On the right-hand side, we have to control $(i, j)$ to use Lemma 2.15 or Lemma 2.16.

Conditions on $(i, j)$ and $(e, f)$ We can take $i$ and $j$ satisfying $1 \leq i \leq j \leq(m+n-1) / 2$. If $i+j$ is odd (then $m+n$ is odd), then we replace $j$ with $m+n-j$ and denote it by $j$ again. Then, as a condition of $(i, j)$, we may assume

$$
1 \leq i \leq j \leq m+n-1,2 \leq i+j \leq m+n-1 \text { and } i+j \text { is even. }
$$

From now on, we regard the equality (4.9) as a controll equivalence between the real value sequences

$$
u \xi^{-\frac{i+j}{2}}\left\{\beta\left|\frac{\xi^{m}-1}{\xi-1}\right|^{2}-\alpha^{\prime}\right\}\left(\xi^{i}-1\right)\left(\xi^{j}-1\right)=\xi^{-m}\left(\xi^{m}-1\right)^{2}
$$


where $u= \pm 1$, or $\pm \xi^{\frac{m+n}{2}}$ only if $m+n$ is even, by Lemma 2.13. We define the integers

$$
e:=\frac{j-i}{2} \text { and } f:=\frac{i+j}{2} \text {. They satisfies } 0 \leq e<f \leq(m+n-1) / 2 .
$$

Using $(e, f)$, we can deform (4.11) as

$$
\begin{aligned}
& u\left\{\beta\left(\xi^{m}+\xi^{-m}\right)-\alpha^{\prime}\left(\xi+\xi^{-1}\right)+2\left(\alpha^{\prime}-\beta\right)\right\}\left\{\left(\xi^{f}+\xi^{-f}\right)-\left(\xi^{e}+\xi^{-e}\right)\right\} \\
& =\left(\xi^{m+1}+\xi^{-(m+1)}\right)-2\left(\xi^{m}+\xi^{-m}\right)+\left(\xi^{m-1}+\xi^{-(m-1)}\right)-2\left(\xi+\xi^{-1}\right)+4
\end{aligned}
$$

We define two symmetric Laurent polynomials

$$
\begin{aligned}
& F(t)=\left\{\beta\left(t^{m}+t^{-m}\right)-\alpha^{\prime}\left(t+t^{-1}\right)+2\left(\alpha^{\prime}-\beta\right)\right\}\left\{\left(t^{f}+t^{-f}\right)-\left(t^{e}+t^{-e}\right)\right\}, \\
& G(t)=\left(t^{m+1}+t^{-(m+1)}\right)-2\left(t^{m}+t^{-m}\right)+\left(t^{m-1}+t^{-(m-1)}\right)-2\left(t+t^{-1}\right)+4,
\end{aligned}
$$

then (4.11) means that two real value sequences (of degree $m+n$ ) induced by $F(t)$ and $G(t)$ are control equivalent, see Subsection 2.3. Note that $F(1)=G(1)=0$. By Lemma 2.16, (4.13) lifts to a congruence of the symmetric Laurent polynomials.

Lemma 4.6 (Necessary condition) Suppose that $\left(A_{m, n} ; \alpha / \beta, 0\right)$ is a lens space. We set $\alpha^{\prime}=\alpha-m n \beta$. Then there exist integers $e$ and $f$ such that $0 \leq e<f \leq(m+n-1) / 2$, and the following congruence holds:

(a) If $m+n$ is odd, we have $F(t) \equiv \pm G(t)\left(\bmod t^{m+n}-1\right)$.

(b) If $m+n$ is even, we have $F(t) \equiv \pm G(t)$ or $F(t) \equiv \pm t^{\frac{m+n}{2}} G(t)\left(\bmod t^{m+n}-1\right)$.

If $m+n$ is even, then $\operatorname{span}(G(t))=2(m+1) \leq m+n$, since the pair $m, n$ is coprime, thus both are odd and $m+2 \leq n$. Furthermore, the congruence also induces an identity

$$
\text { (i) } \operatorname{red}(F(t))= \pm G(t) \quad \text { or } \quad \text { (ii) } \operatorname{red}\left(t^{\frac{m+n}{2}} F(t)\right)= \pm G(t) \text {, }
$$

as in the second half of Lemma 2.16. We will regard it as an equation of $(e, f)$ on the surgery coefficient $\alpha / \beta$ for $M=\left(A_{m, n} ; \alpha / \beta, 0\right)$ to be a lens space: Suppose that $M$ is a lens space, then there exists a solution $(e, f)$ of the equation. We mainly use its contraposition: If the equation has no solution $(e, f)$, then $M$ is not a lens space. The case (b) looks troublesome. To prove that $M$ is not a lens space, we have to show that neither (i) nor (ii) has a solution. Fortunately, we only have to show one of them.

Remark 4.7 In either case $m+n$ is odd or even, to prove that $\left(A_{m, n} ; \alpha / \beta, 0\right)$ is not a lens space, it is sufficient to show that $\operatorname{red}(F(t))= \pm G(t)$ has no solution $(e, f)$, because we can prove the following.

Lemma 4.8 In the case (b) $m+n$ is even in Lemma 4.6, if the equation (i) $\operatorname{red}(F(t))= \pm G(t)$ in (4.15) has a solution, the other equation (ii) $\operatorname{red}\left(t^{\frac{m+n}{2}} F(t)\right)=\mp G(t)$ has a solution, and vice versa. 
Proof We concentrate on the factor $\left\{\left(t^{f}+t^{-f}\right)-\left(t^{e}+t^{-e}\right)\right\}$ of $F(t)$. We transform $(e, f)$ to $\left(e^{\prime}, f^{\prime}\right)$ by

$$
e^{\prime}=\frac{m+n}{2}-f \quad \text { and } \quad f^{\prime}=\frac{m+n}{2}-e,
$$

which satisfies the same condition $0 \leq e^{\prime}<f^{\prime} \leq(m+n-1) / 2$ with (4.12). For a solution $(e, f)$ of the equation $\operatorname{red}(F(t))= \pm G(t)$, its transformation $\left(e^{\prime}, f^{\prime}\right)$ is a solution of $\operatorname{red}\left(t^{\frac{m+n}{2}} F(t)\right)=$ $\mp G(t)$, and vice versa.

In Subsection 5.3, we will prove that $(e, f)=(0,1)$ with $\alpha^{\prime}=0$ is the only solution for the equation $\operatorname{red}(F(t))= \pm G(t)$ in general cases (see Lemma 4.9 (1) below). Note that $\alpha^{\prime}=0$ implies $\alpha / \beta=m n$, which is related to the lens space surgery in Theorem 1.2 .

Using the expression of $R(m, n)$ in Lemma 4.2 (4), we can prove the following.

\section{Lemma 4.9}

(1) The condition $\alpha / \beta=m n$ (i.e. $\alpha^{\prime}=0$ ) is equivalent to $(e, f)=(0,1)$ (i.e. $i=j=1$ ).

(2) In 4.9), if $(e, f)=(0, m)$ (i.e. $i=j=m)$, then there is no root for $\alpha / \beta$.

Proof (1) Suppose that $\alpha / \beta=m n$. Then we have $\tau^{\psi_{d}^{\prime}}(M) \doteq(\xi-1)^{-2}$ by Lemma 4.3, and the equality (4.9) becomes

$$
\frac{1}{(\xi-1)^{2}} \doteq \frac{1}{\left(\xi^{i}-1\right)\left(\xi^{j}-1\right)}
$$

We have $i=j=1$ by the Franz lemma (Lemma 2.10).

Conversely suppose that $i=j=1$. Then the equality (4.8) can be deformed into

$$
\beta R(m, n)-\alpha=u \xi^{-(m-1)}\left(\frac{\xi^{m}-1}{\xi-1}\right)^{2}
$$

where $u= \pm 1$ or $\pm \xi^{\frac{m+n}{2}}$. Using the expression of $R(m, n)$ in Lemma 4.2 (4) and the calculus in the proof of Lemma 4.2 (5), we have

$$
\beta m(n+1)-\alpha+\sum_{j=1}^{m-1} \beta(m-j)\left(\xi^{j}+\xi^{-j}\right)=u\left\{m+\sum_{j=1}^{m-1}(m-j)\left(\xi^{j}+\xi^{-j}\right)\right\} .
$$

By taking the symmetric polynomial lift and Lemma 2.15 (Note that the span is $2(m-1) \leq$ $2([(m+n) / 2]-1))$, the case $u= \pm \xi^{\frac{m+n}{2}}$ does not occur, by Lemma 2.17. We have $\beta=1$ and $\alpha=m n$.

(2) Suppose that $i=j=m$. Then the equality (4.8) is deformed into

$$
\beta R(m, n)-\alpha=u
$$

where $u= \pm 1$ or $\pm \xi^{\frac{m+n}{2}}$. By the same method with above, we have

$$
\beta m(n+1)-\alpha+\sum_{j=1}^{m-1} \beta(m-j)\left(\xi^{j}+\xi^{-j}\right)=u .
$$

By Lemma 2.15 and Lemma 2.17, we have $\beta=0$. Hence there is no root for $\alpha / \beta$. 
Lemma 4.10 Suppose that $\alpha^{\prime}>0$.

(1) $\operatorname{gcd}\left(\alpha^{\prime}, \beta\right)=1$.

(2) If $\alpha^{\prime}=\beta$, then the congruence $F(t) \equiv \pm G(t)$ (mod $\left.t^{m+n}-1\right)$ has a unique solution $(m, n)=(2,3)$.

Proof (1) By the Euclidean algorithm, we have

$$
\operatorname{gcd}\left(\alpha^{\prime}, \beta\right)=\operatorname{gcd}(\alpha-m n \beta, \beta)=\operatorname{gcd}(\alpha, \beta)=1 .
$$

(2) Suppose $\alpha^{\prime}=\beta$. Then, by (1), we have $\alpha^{\prime}=\beta=1$ and the polynomials (4.14) are

$$
\begin{aligned}
& F(t)=t^{-m-f}\left(t^{m+1}-1\right)\left(t^{m-1}-1\right)\left(t^{i}-1\right)\left(t^{j}-1\right), \\
& G(t)=t^{-m-1}\left(t^{m}-1\right)^{2}(t-1)^{2} .
\end{aligned}
$$

The congruence $F(t) \equiv \pm G(t)\left(\bmod t^{m+n}-1\right)$ implies

$$
F(\zeta) \doteq G(\zeta)
$$

where $\zeta$ is a primitive $(m+n)$-th root of unity. Suppose $\operatorname{gcd}(m-1, m+n) \geq 2$ or $\operatorname{gcd}(m+$ $1, m+n) \geq 2$. Then the left-hand side of the equation above is 0 for some $d$. Hence we have $\operatorname{gcd}(m-1, m+n)=1$ and $\operatorname{gcd}(m+1, m+n)=1$. By the Franz lemma [Fz] (Lemma 2.10), we have

$$
\{ \pm(m-1), \pm(m+1), \pm i, \pm j(\bmod m+n)\}=\{ \pm 1, \pm 1, \pm m, \pm m(\bmod m+n)\}
$$

as multiple sets. It has a unique solution $(m, n)=(2,3)$ with $(i, j)=(1,3)$.

\section{Proof of Theorem 1.4 (Lens space surgeries along $A_{m, n}$ )}

The "if part" of Theorem 1.4 (1) follows from Theorem 1.2, thus our main purpose is to prove the "only if part". We study the condition on $\alpha / \beta$ for the equations (4.11) or (4.15)(i) has a solution $(i, j)$ or $(e, f)$, respectively. Our proof is divided into three cases: $m=2$ (Subsection 5.1), $n=m+1$ (Subsection 5.2), and the general case where $m \geq 3$ and $n \geq m+2$ (Subsection [5.3). Note that the first two cases contains the exceptional case $\left(A_{2,3} ; 7,0\right)$. In Subsection 5.4 and 5.5, we prove Theorem 1.4 (2) by using the values of the Reidemeister torsions. We also use Kirby moves.

To make expressions of symmetric Laurent polynomials short, we use the notation $\left\langle t^{x}\right\rangle=$ $t^{x}+t^{-x}$ for any integer $x$. We regard $\left\langle t^{x}\right\rangle$ as $\left\langle t^{-x}\right\rangle$ if $x<0$, and $\left\langle t^{0}\right\rangle=2$. For the terminologies "reduce, reduction (denoted by $\operatorname{red}(P(t))$ )" of symmetric Laurent polynomials, see Definition 2.14. 


\subsection{The case $m=2$}

In this case, $n$ and $m+n=n+2$ are odd. Let $\xi$ denote any $d$-th root of unity, where $d$ is a divisor of $n+2$ with $d \geq 2$. We have $R(2, n)=|\xi+1|^{2}+m n=\xi+\xi^{-1}+m n+2$ by Lemma 4.2 (5), thus the equation (4.11), divided by $\xi^{-1}(\xi-1)^{2}$ as a value sequence, becomes

$$
\xi^{-\frac{i+j-2}{2}} \cdot\left\{\beta\left(\xi+\xi^{-1}\right)-\alpha^{\prime \prime}\right\} \frac{\left(\xi^{i}-1\right)\left(\xi^{j}-1\right)}{(\xi-1)^{2}}=\eta \xi^{-1} \cdot \frac{\left(\xi^{2}-1\right)^{2}}{(\xi-1)^{2}}
$$

where $\alpha^{\prime \prime}=\alpha^{\prime}-2 \beta=\alpha-2(n+1) \beta, \eta= \pm 1$ and $(i, j)$ satisfies the condition (4.10) in the last section. We regard (5.1) as an equality between real value sequences, defined in Subsection 2.3.

(1) The case $2 \leq i+j<n+1$. Note that $i+j$ is even, see (4.10).

By Lemma 2.15, the equalities (5.1) lift to a congruence and

$$
t^{-\frac{i+j-2}{2}} \cdot\left\{\beta\left(t+t^{-1}\right)-\alpha^{\prime \prime}\right\} \frac{\left(t^{i}-1\right)\left(t^{j}-1\right)}{(t-1)^{2}}=\eta t^{-1} \cdot \frac{\left(t^{2}-1\right)^{2}}{(t-1)^{2}} .
$$

Note that $\left(t^{x}-1\right) /(t-1)$ is a polynomial for an integer $x$ and that both hand sides are symmetric Laurent polynomials. The span of the left-hand side is $i+j \leq 2([(m+n) / 2]-1)=$ $2 n$. From $\beta>0$, we have $i=j=1, \beta\left(t+t^{-1}\right)-\alpha^{\prime \prime}=t^{-1}(t+1)^{2}, \beta=1, \alpha^{\prime \prime}=-2$, and $\alpha=2 n$.

(2) The case $i+j=n+1(=2 f$, see (4.12) in Subsection 4.3) $)$.

Then $e=(j-i) / 2$ is an integer satisfying $1 \leq e<\frac{n+1}{2}$. The equation (4.11) is

$$
\xi^{-\frac{n+1}{2}} \cdot\left\{\beta\left(\xi+\xi^{-1}\right)-\alpha^{\prime \prime}\right\}\left(\xi^{i}-1\right)\left(\xi^{j}-1\right)=\eta \xi^{-2} \cdot\left(\xi^{2}-1\right)^{2} .
$$

By Lemma 2.16, it lifts to

$$
\begin{aligned}
& \left(\beta-\alpha^{\prime \prime}\right)\left(t^{\frac{n+1}{2}}+t^{-\frac{n+1}{2}}\right)+\beta\left(t^{\frac{n-1}{2}}+t^{-\frac{n-1}{2}}\right) \\
& -\beta\left(t^{e+1}+t^{-(e+1)}\right)+\alpha^{\prime \prime}\left(t^{e}+t^{-e}\right)-\beta\left(t^{e-1}+t^{-(e-1)}\right) \\
= & \eta\left\{\left(t^{2}+t^{-2}\right)-2\right\},
\end{aligned}
$$

which is, using notations $\left\langle t^{x}\right\rangle=t^{x}+t^{-x}$,

$$
\left(\beta-\alpha^{\prime \prime}\right)\left\langle t^{\frac{n+1}{2}}\right\rangle+\beta\left\langle t^{\frac{n-1}{2}}\right\rangle-\beta\left\langle t^{e+1}\right\rangle+\alpha^{\prime \prime}\left\langle t^{e}\right\rangle-\beta\left\langle t^{e-1}\right\rangle=\eta\left(\left\langle t^{2}\right\rangle-2\right) .
$$

Here we used $\left\langle t^{\frac{n+3}{2}}\right\rangle \equiv\left\langle t^{\frac{n+1}{2}}\right\rangle\left(\bmod t^{n+2}-1\right)$. Note that the span of the left-hand side is at most $n+1=2[(m+n) / 2]$. We have $e=1, \eta=\beta=1$ and $n=3$. Then (5.2) is deformed into

$$
-\alpha^{\prime \prime}\left(t^{2}+t^{-2}\right)+\left(1+\alpha^{\prime \prime}\right)\left(t+t^{-1}\right)-2=\left(t^{2}+t^{-2}\right)-2 .
$$

Hence we have $\alpha^{\prime \prime}=-1$ and $\alpha=7$. In this case, $(i, j)=(1,3)$. This corresponds to the case $\left(A_{2,3} ; 7,0\right)$, and it is a lens space, see Subsection 5.5 . 


\subsection{The case $n=m+1$}

In this case, $m+n=2 m+1$ is odd. Let $\xi$ denote any $d$-th root of unity, where $d$ is a divisor of $2 m+1$ with $d \geq 2$. We use $\zeta=\xi^{m}$ as in Lemma 4.3, then $\xi=\zeta^{-2}$. The Reidemeister torsion in Lemma 4.3 is deformed to

$$
\begin{aligned}
\tau_{d}^{\psi_{d}^{\prime}}(M) & =\xi^{-m}\left\{\beta\left|\frac{\xi^{m}-1}{\xi-1}\right|^{2}-\alpha^{\prime}\right\}\left(\xi^{m}-1\right)^{2}=\zeta\left\{\beta \zeta\left(\frac{\zeta-1}{\zeta^{2}-1}\right)^{2}-\alpha^{\prime}\right\}(\zeta-1)^{-2} \\
& =-\zeta^{2}\left\{\alpha^{\prime} \zeta^{-1}\left(\frac{\zeta^{2}-1}{\zeta-1}\right)^{2}-\beta\right\}\left(\zeta^{2}-1\right)^{-2}=-\zeta^{2}\left\{\alpha^{\prime}\left(\zeta+\zeta^{-1}\right)-\beta\right\}\left(\zeta^{2}-1\right)^{-2} .
\end{aligned}
$$

We apply the same argument on this equality as in Subsection 4.3 and retake $(i, j)$ satisfying the condition (4.10). The equality of the real value sequence (4.11) is

$$
\zeta^{-\frac{i+j}{2}}\left\{\alpha^{\prime} \zeta^{-1}\left(\frac{\zeta^{2}-1}{\zeta-1}\right)^{2}-\beta\right\}\left(\zeta^{i}-1\right)\left(\zeta^{j}-1\right)=\eta \zeta^{-2}\left(\zeta^{2}-1\right)^{2} .
$$

Divided by $\zeta^{-1}(\zeta-1)^{2}$, it induces similar equation to (5.1). We can apply the same argument as in Subsection 5.1. Instead of (5.1), we study

$$
\zeta^{-\frac{i+j-2}{2}} \cdot\left\{\alpha^{\prime}\left(\zeta+\zeta^{-1}\right)-\beta^{\prime}\right\} \frac{\left(\zeta^{i}-1\right)\left(\zeta^{j}-1\right)}{(\zeta-1)^{2}}=\eta \zeta^{-1} \cdot \frac{\left(\zeta^{2}-1\right)^{2}}{(\zeta-1)^{2}}
$$

where $\beta^{\prime}=\beta-2 \alpha^{\prime}(=\beta-2(\alpha-m n \beta))$. Equation (5.3) is obtained from (5.1) by changing $(\xi$ to $\zeta), \beta$ to $\alpha^{\prime}$ and $\alpha^{\prime \prime}$ to $\beta$, respectively. Thus, using the correspondence, we can study their roots by the same argument. In the last subsection, $\beta=0$ was not allowed, but here $\alpha^{\prime}=0$ is allowed (Lemma 4.5).

(1) The case $\alpha^{\prime}=0(\alpha / \beta=m(m+1)=m n)$.

In this case, $\alpha / \beta=m(m+1)$ is a root by Lemma 4.9 (1).

(2) The case $\alpha^{\prime} \geq 1$. The argument is similar to the case $m=2$ in Subsection 5.1 ,

(i) The case $2 \leq i+j \leq 2 m-2$.

There is no root for $\alpha^{\prime}, \beta$, by the argument with (1) in Subsection 5.1, since the corresponding root $\left(\alpha^{\prime}, \beta^{\prime}\right)=(1,-2)$ implies $\beta=0$, which is not allowed.

(ii) The case $i+j=2 m$.

Then $e=(j-i) / 2$ is an integer satisfying $1 \leq e<m$. The argument is similar to that of (2) in Subsection [5.1. We have

$$
\left(\alpha^{\prime}-\beta^{\prime}\right)\left\langle t^{m}\right\rangle+\alpha^{\prime}\left\langle t^{m-1}\right\rangle-\alpha^{\prime}\left\langle t^{e+1}\right\rangle+\beta^{\prime}\left\langle t^{e}\right\rangle-\alpha^{\prime}\left\langle t^{e-1}\right\rangle=\eta\left(\left\langle t^{2}\right\rangle-2\right)
$$

Here we used $\left\langle t^{m+1}\right\rangle \equiv\left\langle t^{m}\right\rangle\left(\bmod t^{2 m+1}-1\right)$. The span of the left-hand side is at most $2 m=2[(m+n) / 2]$. Corresponding to that the equation (5.1) has a root $(\beta, \alpha)=(-1,1)$, this equation has a root $\left(\alpha^{\prime}, \beta^{\prime}\right)=(1,-1)$ only if $m=2$, which implies $(\alpha, \beta)=(7,1)$. This corresponds to the case $\left(A_{2,3} ; 7,0\right)$, and it is a lens space, see Subsection 5.5 . 


\subsection{The case $m \geq 3$ and $n \geq m+2$}

Let $P(t)$ be a symmetric Laurent polynomial in Definition 2.14 of the form:

$$
P(t)=a_{0}+\sum_{i=1}^{\infty} a_{i}\left(t^{i}+t^{-i}\right)=a_{0}+\sum_{i=1}^{\infty} a_{i}\left\langle t^{i}\right\rangle .
$$

We call $a_{0}$ the constant term, $a_{i}\left\langle t^{i}\right\rangle$ the $i$-th term, and $a_{i}$ the $i$-th coefficient. When $P(t)$ is considered in $\mathbb{Q}\left[t, t^{-1}\right] /\left(t^{N}-1\right), P(t)$ (as above) is reduced if $a_{i}=0$ for all $i>[N / 2]$. We denote the reduction of $P(t)$ by $\operatorname{red}(P(t))$.

Assumption (of this subsection) Let $(m, n)$ be a fixed pair of positive coprime integers with $m \geq 3$ and $n \geq m+2$. We assume that $\beta \geq 1, \alpha^{\prime}=\alpha-m n \beta \geq 0$, see Lemma 4.5. We set $F(t)$ and $G(t)$ as:

$$
\begin{aligned}
& F(t)=\left\{\beta\left\langle t^{m}\right\rangle-\alpha^{\prime}\left\langle t^{1}\right\rangle+2\left(\alpha^{\prime}-\beta\right)\right\}\left\{\left\langle t^{f}\right\rangle-\left\langle t^{e}\right\rangle\right\} \\
& G(t)=\left\langle t^{m+1}\right\rangle-2\left\langle t^{m}\right\rangle+\left\langle t^{m-1}\right\rangle-2\left\langle t^{1}\right\rangle+4
\end{aligned}
$$

in (4.14) in the last section. From now on, we fix $N=m+n$. Note that $G(t)$ is already reduced. Let

$$
\operatorname{red}(F(t))= \pm G(t)
$$

be an equation on $(e, f)$. Existence of an integral solution is a necessary condition for $\left(A_{m, n} ; \alpha / \beta, 0\right)$ to be a lens space. We show that there exist no integral solution of (5.4) with $0 \leq e<f \leq(m+n-1) / 2$ under $\alpha^{\prime}>0$, see Lemma 4.9 (1) for the case $\alpha^{\prime}=0$. By Lemma 4.10, we assume that $\alpha^{\prime} \neq \beta$.

Using $\left\langle t^{a}\right\rangle \cdot\left\langle t^{b}\right\rangle=\left\langle t^{a+b}\right\rangle+\left\langle t^{a-b}\right\rangle$, we have

$$
\begin{aligned}
F(t)= & \beta\left\langle t^{m+f}\right\rangle+\beta\left\langle t^{m-f}\right\rangle-\alpha^{\prime}\left\langle t^{f+1}\right\rangle-\alpha^{\prime}\left\langle t^{f-1}\right\rangle+2\left(\alpha^{\prime}-\beta\right)\left\langle t^{f}\right\rangle \\
& -\beta\left\langle t^{m+e}\right\rangle-\beta\left\langle t^{m-e}\right\rangle+\alpha^{\prime}\left\langle t^{e+1}\right\rangle+\alpha^{\prime}\left\langle t^{e-1}\right\rangle-2\left(\alpha^{\prime}-\beta\right)\left\langle t^{e}\right\rangle .
\end{aligned}
$$

This consists of non-zero ten terms, but may not be reduced. On the other hand, $G(t)$ has five terms and is already reduced. Thus our problem is "Which term in $F(t)$ is reduced to a term in $G(t)$ ?" and "Which terms in $F(t)$ are cancelled with other terms?" On the terms $\left\langle t^{m+f}\right\rangle,\left\langle t^{m-f}\right\rangle,\left\langle t^{m+e}\right\rangle$ and $\left\langle t^{m-e}\right\rangle$, we have

$$
\operatorname{red}\left(\left\langle t^{m+x}\right\rangle\right)=\left\{\begin{array}{ll}
\left\langle t^{m+x}\right\rangle & \text { if } x \leq(n-m) / 2 \\
\left\langle t^{n-x}\right\rangle & \text { if } x>(n-m) / 2
\end{array}, \quad \operatorname{red}\left(\left\langle t^{m-x}\right\rangle\right)=\left\langle t^{|m-x|}\right\rangle,\right.
$$

for $x=e$ or $f$ (thus $0 \leq x \leq(m+n-1) / 2)$. The term $\left\langle t^{f+1}\right\rangle$ is already reduced except only one case:

Case $\mathbf{F}$ If $(m+n)$ is odd and $f=(m+n-1) / 2$, it holds that $\operatorname{red}\left(\left\langle t^{f+1}\right\rangle\right)=\left\langle t^{f}\right\rangle$.

In Case F, it holds that $f-m=(n-m-1) / 2 \geq 1 / 2$, thus we have:

Lemma 5.1 In Case $F$, it holds that $f \geq m+1$. Furthermore, for an integer $a \geq 1$, $f=m+a$ is equivalent to $n=m+2 a+1$. 
We will often take care of this exceptional case. The other five terms are already reduced. It is easy to see:

\section{Lemma 5.2}

(1) Neither $\left\langle t^{m+x}\right\rangle$ nor $\left\langle t^{x+1}\right\rangle$ can be reduced to the constant term.

(2) The term $\left\langle t^{2 m}\right\rangle\left(\left\langle t^{m+x}\right\rangle\right.$ with $\left.x=m\right)$ can be reduced to neither the constant term nor the 1-st term.

The graph of the degrees of $\operatorname{red}\left(\left\langle t^{m+x}\right\rangle\right), \operatorname{red}\left(\left\langle t^{m-x}\right\rangle\right), \operatorname{red}\left(\left\langle t^{x+1}\right\rangle\right)$ and $\operatorname{red}\left(\left\langle t^{x-1}\right\rangle\right)$ are useful, see Figure 4. We are interested in only the points whose coordinates are integers. We set the rectangle formed by $y=\operatorname{deg}\left(\operatorname{red}\left(\left\langle t^{m+x}\right\rangle\right)\right)$ and $y=\operatorname{deg}\left(\operatorname{red}\left(\left\langle t^{m-x}\right\rangle\right)\right)$ as $R$. The intersection

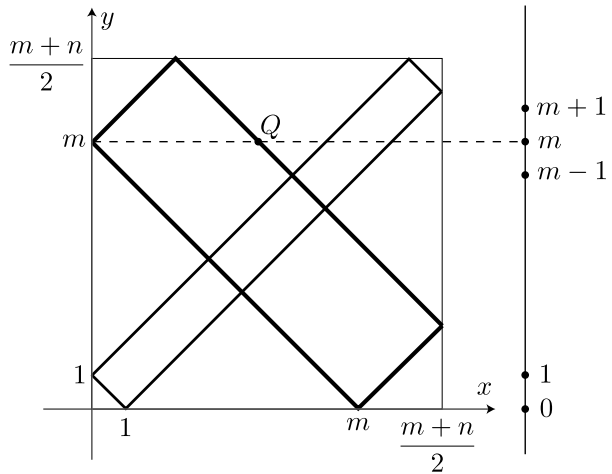

(a) $n<3 m$

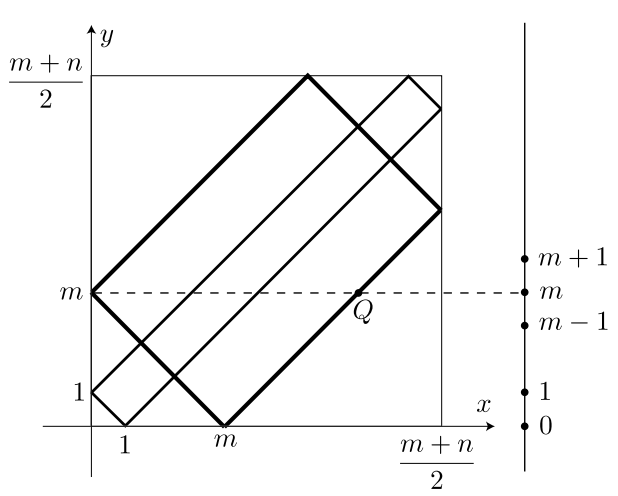

(b) $n>3 m$

Figure 4: Graph of the degrees I

points of $R$ and the line $y=k$ correspond to solve the equation $\operatorname{red}\left(\left\langle t^{m \pm x}\right\rangle\right)=\left\langle t^{k}\right\rangle$. The point $Q$ is one of the intersections for $k=m$ other than $(0, m)$. The $(x, y)$-coordinate of $Q$ is as follows:

If $n<3 m$, then $Q(n-m, m)$, which corresponds to $\operatorname{red}\left(\left\langle t^{m+x}\right\rangle\right)=\left\langle t^{m}\right\rangle$,

If $n>3 m$, then $Q(2 m, m)$, which corresponds to $\operatorname{red}\left(\left\langle t^{m-x}\right\rangle\right)=\left\langle t^{m}\right\rangle$.

Here, note that $n=3 m$ contradicts coprimeness of $m$ and $n$.

Lemma 5.3 (on the case $\operatorname{red}\left(\left\langle t^{m \pm x}\right\rangle\right)=\left\langle t^{m}\right\rangle$ )

By $x_{Q}$, we denote the $x$-coordinate $(=n-m$ or $2 m)$ of $Q$. Then we have

(A) $x_{Q} \neq m$,

(B) $x_{Q}=m+a$ only if $n=2 m+a$, for $a=-2,-1,1$ or 2 ,

(C) $x_{Q}=a$ only if $n=m+a$, for $a=2$ or 3 .

Since $G(t)$ has the non-zero constant term, by Lemma 5.2 (1), we have

$$
\{e, f\} \cap\{0,1, m\} \neq \emptyset .
$$

The proof is divided into the five cases: (1) $e=0$, (2) $e=1$ with $m \geq 4$, (3) $e=m$, (4) " $e \neq 0,1$ and $f=m "$, and (5) $e=1$ with $m=3$. Note that $f=0$ is impossible and that the case $f=1$ is included by the case $e=0$, because of the assumption $e<f$. 
Case $1(e=0)$

$$
\begin{aligned}
F(t)= & \beta\left\langle t^{m+f}\right\rangle+\beta\left\langle t^{m-f}\right\rangle-\alpha^{\prime}\left\langle t^{f+1}\right\rangle-\alpha^{\prime}\left\langle t^{f-1}\right\rangle+2\left(\alpha^{\prime}-\beta\right)\left\langle t^{f}\right\rangle \\
& -2 \beta\left\langle t^{m}\right\rangle+2 \alpha^{\prime}\left\langle t^{1}\right\rangle-4\left(\alpha^{\prime}-\beta\right) .
\end{aligned}
$$

Since $G(t)$ has a non-trivial $(m+1)$ - and $(m-1)$-term, by Lemma 5.3 , we have (caring Case F)

$$
f \in\left\{1, x_{Q}+\epsilon, m, m+1, m+2\right\} \cap\left\{1, x_{Q}-\epsilon, m-2, m-1, m\right\},
$$

where $\epsilon=-1$ if $n<3 m$ (and $\epsilon=+1$ if $n>3 m$, respectively). Figure 5 helps to understand

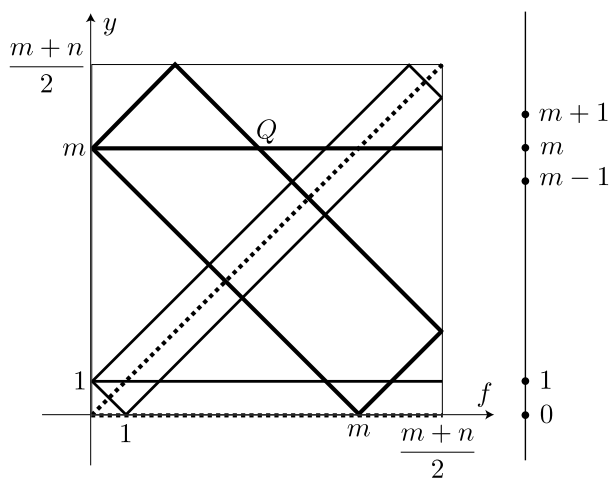

(a) $n<3 m$

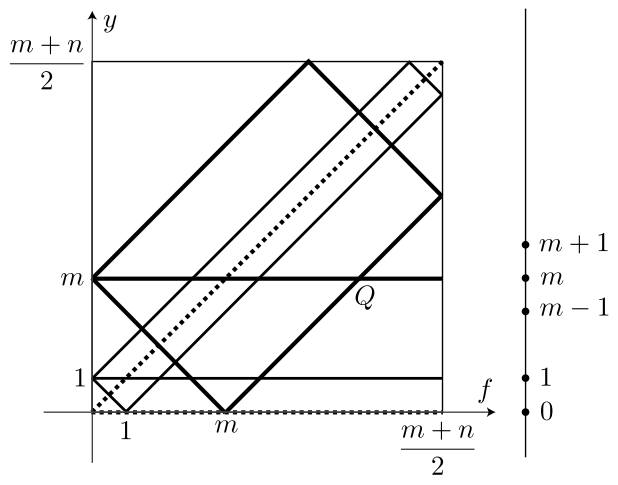

(b) $n>3 m$

Figure 5: Graph of the degrees II

it. Thus we have four cases: (i) $f=1$, (ii) $f=m$, (iii) $f=m+2\left(\right.$ if $n=2 m+1\left(x_{Q}=m+1\right)$ ) or (iv) $f=m-2$ (if $n=2 m-1\left(x_{Q}=m-1\right)$ ) by Lemma 5.3 (B).

In each case, Case $\mathrm{F}$ (i.e., $\left.\operatorname{red}\left(\left\langle t^{f+1}\right\rangle\right)=\left\langle t^{f}\right\rangle\right)$ does not occur because $f \leq m$ in (i), (ii) and (iv), and because $f \neq(m+n-1) / 2$ in (iii), by Lemma 5.1 .

Subcase(1-i): $(e, f)=(0,1)$.

$$
F(t)=\beta\left\langle t^{m+1}\right\rangle-2 \beta\left\langle t^{m}\right\rangle+\beta\left\langle t^{m-1}\right\rangle-\alpha^{\prime}\left\langle t^{2}\right\rangle+2\left(2 \alpha^{\prime}-\beta\right)\left\langle t^{1}\right\rangle-2\left(3 \alpha^{\prime}-2 \beta\right) .
$$

Since $\alpha^{\prime} \neq 0$, to cancel $-\alpha^{\prime}\left\langle t^{2}\right\rangle$, we need $m=3$ and $\alpha^{\prime}=\beta$, which contradicts the assumption. (If we admit $\alpha^{\prime}=0$, then $(e, f)=(0,1)$ is a solution, see Lemma 4.9 (1).)

Subcase(1-ii): $(e, f)=(0, m)$.

$$
F(t)=\beta\left\langle t^{2 m}\right\rangle-\alpha^{\prime}\left\langle t^{m+1}\right\rangle+2\left(\alpha^{\prime}-2 \beta\right)\left\langle t^{m}\right\rangle-\alpha^{\prime}\left\langle t^{m-1}\right\rangle+2 \alpha^{\prime}\left\langle t^{1}\right\rangle-2\left(2 \alpha^{\prime}-3 \beta\right) .
$$

Since $2 m>m+1$, the $2 m$-th term is not reduced. By Lemma $5.2(2)$, and the ratio of the $(m+1)$-th and the $(m-1)$-th coefficients $(=1)$, we have $\operatorname{red}\left(\left\langle t^{2 m}\right\rangle\right)=\left\langle t^{m}\right\rangle$ and $n-m=m$. It contradicts coprimeness of $m$ and $n$.

Subcase(1-iii): $(e, f)=(0, m+2)$ with $n=2 m+1$. It holds that $\operatorname{red}\left(\left\langle t^{2 m+2}\right\rangle\right)=\left\langle t^{m-1}\right\rangle$.

$$
\begin{aligned}
F(t)= & -\alpha^{\prime}\left\langle t^{m+3}\right\rangle+2\left(\alpha^{\prime}-\beta\right)\left\langle t^{m+2}\right\rangle \\
& -\alpha^{\prime}\left\langle t^{m+1}\right\rangle-2 \beta\left\langle t^{m}\right\rangle+\beta\left\langle t^{m-1}\right\rangle+\beta\left\langle t^{2}\right\rangle+2 \alpha^{\prime}\left\langle t^{1}\right\rangle-4\left(\alpha^{\prime}-\beta\right) .
\end{aligned}
$$


Since $m \geq 3$ (thus $m+2 \leq(m+n) / 2)$ and $\alpha^{\prime} \neq \beta$, the $(m+2)$-th term cannot be canceled. We have a contradiction.

Subcase(1-iv): $(e, f)=(0, m-2)$ with $n=2 m-1$. If $m=3$, we go back to (1-i), thus we assume $m \geq 4$. It holds that $\operatorname{red}\left(\left\langle t^{2 m-2}\right\rangle\right)=\left\langle t^{m+1}\right\rangle$.

$$
\begin{aligned}
F(t)= & \beta\left\langle t^{m+1}\right\rangle-2 \beta\left\langle t^{m}\right\rangle-\alpha^{\prime}\left\langle t^{m-1}\right\rangle \\
& +2\left(\alpha^{\prime}-\beta\right)\left\langle t^{m-2}\right\rangle-\alpha^{\prime}\left\langle t^{m-3}\right\rangle+\beta\left\langle t^{2}\right\rangle+2 \alpha^{\prime}\left\langle t^{1}\right\rangle-4\left(\alpha^{\prime}-\beta\right) .
\end{aligned}
$$

All terms are already reduced. By the signs of the $(m+1)$-th and the $(m-1)$-th coefficients, we have a contradiction.

Case $2(e=1$ with $m \geq 4)$

$$
\begin{aligned}
F(t)= & \beta\left\langle t^{m+f}\right\rangle+\beta\left\langle t^{m-f}\right\rangle-\alpha^{\prime}\left\langle t^{f+1}\right\rangle-\alpha^{\prime}\left\langle t^{f-1}\right\rangle+2\left(\alpha^{\prime}-\beta\right)\left\langle t^{f}\right\rangle \\
& -\beta\left\langle t^{m+1}\right\rangle-\beta\left\langle t^{m-1}\right\rangle+\alpha^{\prime}\left\langle t^{2}\right\rangle-2\left(\alpha^{\prime}-\beta\right)\left\langle t^{1}\right\rangle+2 \alpha^{\prime} .
\end{aligned}
$$

Recall the assumption $f>e=1$. Comparing $F(t)$ and $G(t)$, we have that at least one term

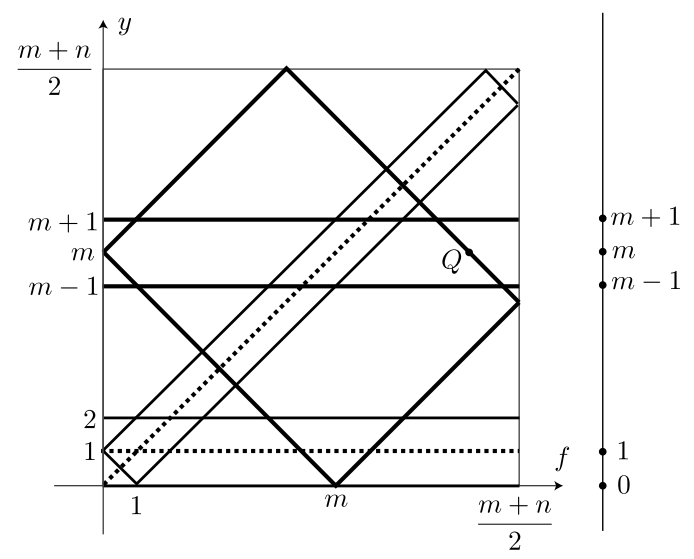

Figure 6: Graph of the degrees III

in $F(t)$ is equal to or reduced to the $m$-th term, and that the term $\alpha^{\prime}\left\langle t^{2}\right\rangle$ is canceled with another term whose coefficient is negative. Here we used $m \geq 4$ (i.e., $\left\langle t^{m-1}\right\rangle \neq\left\langle t^{2}\right\rangle$ ). We have (caring Case F)

$$
f \in\left\{m-1, m, m+1, x_{Q}\right\} \cap\{2,3\},
$$

where $x_{Q}$ is the value defined in Lemma 5.3. Figure 6 helps to understand it. Since $f \leq 3$, Case $\mathrm{F}$ does not occur by Lemma 5.1. Thus we have three cases: (i) $m=4$ and $f=3$, (ii) $f=2$ (if $n=m+2\left(x_{Q}=2\right)$ ), (iii) $f=3$ (if $n=m+3\left(x_{Q}=3\right)$ ), see Lemma 5.3 (C).

Subcase(2-i): $m=4$ and $(e, f)=(1,3)$.

$$
F(t)=\beta\left\langle t^{7}\right\rangle-\beta\left\langle t^{5}\right\rangle-\alpha^{\prime}\left\langle t^{4}\right\rangle+\left(2 \alpha^{\prime}-3 \beta\right)\left\langle t^{3}\right\rangle-\left(2 \alpha^{\prime}-3 \beta\right)\left\langle t^{1}\right\rangle+2 \alpha^{\prime} .
$$

The term $\beta\left\langle t^{7}\right\rangle$ has to be reduced: $\operatorname{red}\left(\left\langle t^{7}\right\rangle\right)=\left\langle t^{n-3}\right\rangle$ and $3 \leq n-3 \leq 5$. By coprimeness of $m$ and $n$, we have $n=7$. By the 5 -th and the 4 -th coefficients, we have a contradiction. 
Subcase(2-ii): $m \geq 4, f=2$ (if $n=m+2\left(x_{Q}=2\right)$ ). It holds that $\operatorname{red}\left(\left\langle t^{m+2}\right\rangle\right)=\left\langle t^{m}\right\rangle$.

$F(t)=-\beta\left\langle t^{m+1}\right\rangle+\beta\left\langle t^{m}\right\rangle-\beta\left\langle t^{m-1}\right\rangle+\beta\left\langle t^{m-2}\right\rangle-\alpha^{\prime}\left\langle t^{3}\right\rangle+\left(3 \alpha^{\prime}-2 \beta\right)\left\langle t^{2}\right\rangle-\left(3 \alpha^{\prime}-2 \beta\right)\left\langle t^{1}\right\rangle+2 \alpha^{\prime}$.

All terms are already reduced. Considering the ratio of the $(m+1)$-st and the $m$-th coefficients, we have a contradiction.

Subcase(2-iii): $m \geq 4, f=3$ (if $n=m+3\left(x_{Q}=3\right)$ ). It holds that $\operatorname{red}\left(\left\langle t^{m+3}\right\rangle\right)=\left\langle t^{m}\right\rangle$.

$F(t)=-\beta\left\langle t^{m+1}\right\rangle+\beta\left\langle t^{m}\right\rangle-\beta\left\langle t^{m-1}\right\rangle+\beta\left\langle t^{m-3}\right\rangle-\alpha^{\prime}\left\langle t^{4}\right\rangle+2\left(\alpha^{\prime}-\beta\right)\left\langle t^{3}\right\rangle-2\left(\alpha^{\prime}-\beta\right)\left\langle t^{1}\right\rangle+2 \alpha^{\prime}$

All terms are already reduced. If $m \geq 5$, then we have a contradiction by the same method with the last case. If $m=4$,

$$
\operatorname{red}(F(t))=-\beta\left\langle t^{5}\right\rangle-\left(\alpha^{\prime}-\beta\right)\left\langle t^{4}\right\rangle+\left(2 \alpha^{\prime}-3 \beta\right)\left\langle t^{3}\right\rangle-\left(2 \alpha^{\prime}-3 \beta\right)\left\langle t^{1}\right\rangle+2 \alpha^{\prime} .
$$

Considering the ratio of the first two coefficients, $-\beta:-\left(\alpha^{\prime}-\beta\right)=1:-2$, i.e., $\beta=-\alpha^{\prime}<0$. We have a contradiction.

In the rest of the proof, we will often use the following:

Lemma 5.4 Even if $\operatorname{red}\left(\left\langle t^{a}\right\rangle\right)=\left\langle t^{b}\right\rangle$, the sum $\pm \beta\left\langle t^{a}\right\rangle \pm \alpha^{\prime}\left\langle t^{b}\right\rangle$ of any signs never cancel by the reduction.

This lemma is easily shown by the assumption $\alpha^{\prime}>0, \beta>0$ and $\alpha^{\prime} \neq \beta$.

Case $3(e=m)$

$$
\begin{aligned}
F(t)= & \beta\left\langle t^{m+f}\right\rangle+\beta\left\langle t^{m-f}\right\rangle-\alpha^{\prime}\left\langle t^{f+1}\right\rangle-\alpha^{\prime}\left\langle t^{f-1}\right\rangle+2\left(\alpha^{\prime}-\beta\right)\left\langle t^{f}\right\rangle \\
& -\beta\left\langle t^{2 m}\right\rangle-2 \beta+\alpha^{\prime}\left\langle t^{m+1}\right\rangle+\alpha^{\prime}\left\langle t^{m-1}\right\rangle-2\left(\alpha^{\prime}-\beta\right)\left\langle t^{m}\right\rangle .
\end{aligned}
$$

Recall the assumption $f>e=m$. Comparing $F(t)$ and $G(t)$, at least one term in $F(t)$ is equal to or reduced to the 1-st term. By Lemma 5.2 (2), we have two cases: (i) $f=m+1$ (It can be in Case F) and (ii) $\operatorname{red}\left(\left\langle t^{m+f}\right\rangle\right)=\left\langle t^{1}\right\rangle$. But in the latter case, $f=n-1 \leq(m+n) / 2$ (see the graph in Figure 44), thus $n=m+2$ and $f=m+1$. We only have to study the case (i).

$\operatorname{Subcase}(3-\mathrm{i}):(e, f)=(m, m+1)$.

$$
\begin{aligned}
F(t)= & \beta\left\langle t^{2 m+1}\right\rangle-\beta\left\langle t^{2 m}\right\rangle-\alpha^{\prime}\left\langle t^{m+2}\right\rangle \\
& +\left(3 \alpha^{\prime}-2 \beta\right)\left\langle t^{m+1}\right\rangle-\left(3 \alpha^{\prime}-2 \beta\right)\left\langle t^{m}\right\rangle+\alpha^{\prime}\left\langle t^{m-1}\right\rangle+\beta\left\langle t^{1}\right\rangle-2 \beta .
\end{aligned}
$$

We focus on $-\alpha^{\prime}\left\langle t^{m+2}\right\rangle$. Neither $\beta\left\langle t^{2 m+1}\right\rangle-\alpha^{\prime}\left\langle t^{m+2}\right\rangle$ nor $-\beta\left\langle t^{2 m}\right\rangle-\alpha^{\prime}\left\langle t^{m+2}\right\rangle$ cancel by the reduction by Lemma 5.4. Thus, if $m+2 \leq(m+n) / 2$ (i.e., $n \geq m+4)$, the term $-\alpha^{\prime}\left\langle t^{m+2}\right\rangle$ is left after the reduction, which is a contradiction. We have $n=m+2$ or $n=m+3$.

First we assume $n=m+2$, then $\operatorname{red}\left(\beta\left\langle t^{2 m+1}\right\rangle-\beta\left\langle t^{2 m}\right\rangle-\alpha^{\prime}\left\langle t^{m+2}\right\rangle\right)=\beta\left\langle t^{1}\right\rangle-\beta\left\langle t^{2}\right\rangle-\alpha^{\prime}\left\langle t^{m}\right\rangle$. Considering the ratio of the $(m+1)$-th and the $(m-1)$-th coefficient, we need $\alpha^{\prime}=\beta$. We have a contradiction. Next we assume $n=m+3$, which implies $m \geq 4$. Then $\operatorname{red}\left(\beta\left\langle t^{2 m+1}\right\rangle-\right.$ $\left.\beta\left\langle t^{2 m}\right\rangle-\alpha^{\prime}\left\langle t^{m+2}\right\rangle\right)=\beta\left\langle t^{2}\right\rangle-\beta\left\langle t^{3}\right\rangle-\alpha^{\prime}\left\langle t^{m+1}\right\rangle$. (It is in Case F.) The second coefficient $\beta \neq 0$, we have a contradiction. 
Case $4(f=m)$ It is not in Case F by Lemma 5.1 ,

$$
\begin{aligned}
F(t)= & -\beta\left\langle t^{m+e}\right\rangle-\beta\left\langle t^{m-e}\right\rangle+\alpha^{\prime}\left\langle t^{e+1}\right\rangle+\alpha^{\prime}\left\langle t^{e-1}\right\rangle-2\left(\alpha^{\prime}-\beta\right)\left\langle t^{e}\right\rangle \\
& +\beta\left\langle t^{2 m}\right\rangle+2 \beta-\alpha^{\prime}\left\langle t^{m+1}\right\rangle-\alpha^{\prime}\left\langle t^{m-1}\right\rangle+2\left(\alpha^{\prime}-\beta\right)\left\langle t^{m}\right\rangle .
\end{aligned}
$$

Recall the assumption $e<f=m$. Comparing $F(t)$ and $G(t)$, we have that at least one term in $F(t)$ is equal to or reduced to the 1-st term. By Lemma $5.2(2),\left\langle t^{2 m}\right\rangle$ is not reduced to the 1-st term. It does not hold that $\operatorname{red}\left(\left\langle t^{m+e}\right\rangle\right)=\left\langle t^{1}\right\rangle$, because it implies $e=n-1$, which contradicts to $e<f=m$. Thus we need $\operatorname{red}\left(\left\langle t^{m-e}\right\rangle\right)=\left\langle t^{1}\right\rangle$, i.e., $e=m-1$.

$$
\begin{aligned}
F(t)= & \beta\left\langle t^{2 m}\right\rangle-\beta\left\langle t^{2 m-1}\right\rangle \\
& -\alpha^{\prime}\left\langle t^{m+1}\right\rangle+\left(3 \alpha^{\prime}-2 \beta\right)\left\langle t^{m}\right\rangle-\left(3 \alpha^{\prime}-2 \beta\right)\left\langle t^{m-1}\right\rangle+\alpha^{\prime}\left\langle t^{m-2}\right\rangle-\beta\left\langle t^{1}\right\rangle+2 \beta .
\end{aligned}
$$

One of the first two terms has to cancel $\alpha^{\prime}\left\langle t^{m-2}\right\rangle$, but it is impossible, because neither $\beta\left\langle t^{2 m}\right\rangle+$ $\alpha^{\prime}\left\langle t^{m-2}\right\rangle$ nor $-\beta\left\langle t^{2 m-1}\right\rangle+\alpha^{\prime}\left\langle t^{m-2}\right\rangle$ cancel by Lemma 5.4.

Case $5(e=1$ with $m=3)$ Note that $G(t)=\left\langle t^{4}\right\rangle-2\left\langle t^{3}\right\rangle+\left\langle t^{2}\right\rangle-2\left\langle t^{1}\right\rangle+4$.

$$
\begin{aligned}
F(t)= & \beta\left\langle t^{3+f}\right\rangle+\beta\left\langle t^{3-f}\right\rangle-\alpha^{\prime}\left\langle t^{f+1}\right\rangle-\alpha^{\prime}\left\langle t^{f-1}\right\rangle+2\left(\alpha^{\prime}-\beta\right)\left\langle t^{f}\right\rangle \\
& -\beta\left\langle t^{4}\right\rangle+\left(\alpha^{\prime}-\beta\right)\left\langle t^{2}\right\rangle-2\left(\alpha^{\prime}-\beta\right)\left\langle t^{1}\right\rangle+2 \alpha^{\prime} .
\end{aligned}
$$

The proof is divided into four cases: (i) $f=2$, (ii) $f=3$, (iii) $f=4$ or (iv) $f \geq 5$.

Subcase(5-i): $(e, f)=(1,2)$.

$$
F(t)=\beta\left\langle t^{5}\right\rangle-\beta\left\langle t^{4}\right\rangle-\alpha^{\prime}\left\langle t^{3}\right\rangle+3\left(\alpha^{\prime}-\beta\right)\left\langle t^{2}\right\rangle-3\left(\alpha^{\prime}-\beta\right)\left\langle t^{1}\right\rangle+2 \alpha^{\prime}
$$

The term $\beta\left\langle t^{5}\right\rangle$ has to be reduced: $\operatorname{red}\left(\left\langle t^{5}\right\rangle\right)=\left\langle t^{n-2}\right\rangle$ and $n-2 \geq m \geq 3$. Considering the ratio of the 1-st coefficient and the constant term, we have $-3\left(\alpha^{\prime}-\beta\right): 2 \alpha^{\prime}=-2: 4$, i.e., $2 \alpha^{\prime}=3 \beta$. Considering the ratio of the 2 -nd and the 1 -st coefficients, we have a contradiction.

Subcase(5-ii): $(e, f)=(1,3)$.

$$
F(t)=\beta\left\langle t^{6}\right\rangle-\left(\alpha^{\prime}+\beta\right)\left\langle t^{4}\right\rangle+2\left(\alpha^{\prime}-\beta\right)\left\langle t^{3}\right\rangle-\beta\left\langle t^{2}\right\rangle-2\left(\alpha^{\prime}-\beta\right)\left\langle t^{1}\right\rangle+2\left(\alpha^{\prime}+\beta\right)
$$

The term $\beta\left\langle t^{6}\right\rangle$ has to be reduced: $\operatorname{red}\left(\left\langle t^{6}\right\rangle\right)=\left\langle t^{n-3}\right\rangle$ and $n-3 \geq 2$. Considering the ratio of the 1-st coefficient and the constant term, we have $-2\left(\alpha^{\prime}-\beta\right): 2\left(\alpha^{\prime}+\beta\right)=-2: 4$, i.e., $\alpha^{\prime}=3 \beta$.

$$
F(t)=\beta \cdot\left(\left\langle t^{6}\right\rangle-4\left\langle t^{4}\right\rangle+4\left\langle t^{3}\right\rangle-\left\langle t^{2}\right\rangle-4\left\langle t^{1}\right\rangle+8\right) .
$$

In any cases $\operatorname{red}\left(\left\langle t^{6}\right\rangle\right)=\left\langle t^{4}\right\rangle,\left\langle t^{3}\right\rangle$ or $\left\langle t^{2}\right\rangle$, it does not hold that $\operatorname{red}(F(t))= \pm G(t)$. We have a contradiction.

Subcase(5-iii): $(e, f)=(1,4)$.

$$
F(t)=\beta\left\langle t^{7}\right\rangle-\alpha^{\prime}\left\langle t^{5}\right\rangle+\left(2 \alpha^{\prime}-3 \beta\right)\left\langle t^{4}\right\rangle-\alpha^{\prime}\left\langle t^{3}\right\rangle+\left(\alpha^{\prime}-\beta\right)\left\langle t^{2}\right\rangle-\left(2 \alpha^{\prime}-3 \beta\right)\left\langle t^{1}\right\rangle+2 \alpha^{\prime} .
$$

By Lemma 5.4, both terms $\beta\left\langle t^{7}\right\rangle$ and $-\alpha^{\prime}\left\langle t^{5}\right\rangle$ have to be reduced. It is possible only if $(m+n) / 2<5$, i.e., $n<7$. Since $n \geq m+2=5$, and $n$ is coprime to $m=3$, we have $n=5$. Then $\operatorname{red}\left(\left\langle t^{7}\right\rangle\right)=\left\langle t^{1}\right\rangle$ and $\operatorname{red}\left(\left\langle t^{5}\right\rangle\right)=\left\langle t^{3}\right\rangle$. Considering the ratio of the 4 -th and the 2-nd 
coefficients, we have $\left(2 \alpha^{\prime}-3 \beta\right)=\left(\alpha^{\prime}-\beta\right)$, i.e., $\alpha^{\prime}=2 \beta$. In this case, we lose the 1 -st term: $\operatorname{red}\left(\beta\left\langle t^{7}\right\rangle-\left(2 \alpha^{\prime}-3 \beta\right)\left\langle t^{1}\right\rangle\right)=0$. We have a contradiction.

Subcase(5-iv): $e=1$ and $f \geq 5$.

$$
\begin{aligned}
F(t)= & \beta\left\langle t^{f+3}\right\rangle-\alpha^{\prime}\left\langle t^{f+1}\right\rangle+2\left(\alpha^{\prime}-\beta\right)\left\langle t^{f}\right\rangle \\
& -\alpha^{\prime}\left\langle t^{f-1}\right\rangle+\beta\left\langle t^{f-3}\right\rangle-\beta\left\langle t^{4}\right\rangle+\left(\alpha^{\prime}-\beta\right)\left\langle t^{2}\right\rangle-2\left(\alpha^{\prime}-\beta\right)\left\langle t^{1}\right\rangle+2 \alpha^{\prime} .
\end{aligned}
$$

The $f$-th term has to be cancelled. If it is cancelled by the $(f+3)$-th term, then the $(f+1)$-th term is left after the reduction. If it is cancelled by the $(f+1)$-th term, which is in Case $\mathrm{F}$, we have $\alpha^{\prime}=2 \beta, n=2 f-2$, and $\operatorname{red}\left(\left\langle t^{f+3}\right\rangle\right)=\left\langle t^{f-2}\right\rangle$ with $f-2 \geq 3$, thus

$$
\operatorname{red}(F(t))=\beta \cdot\left(-2\left\langle t^{f-1}\right\rangle+\left\langle t^{f-2}\right\rangle+\left\langle t^{f-3}\right\rangle-\left\langle t^{4}\right\rangle+\left\langle t^{2}\right\rangle-2\left\langle t^{1}\right\rangle+4\right) .
$$

If $f>5$, then the top degree is $f-1>4$, we have a contradiction. Otherwise $f=5$, we have

$$
\operatorname{red}(F(t))=\beta \cdot\left(-3\left\langle t^{4}\right\rangle+\left\langle t^{3}\right\rangle+2\left\langle t^{2}\right\rangle-2\left\langle t^{1}\right\rangle+4\right) .
$$

The ratio of the coefficients is different from that of $G(t)$. We have a contradiction.

In any cases, we have a contradiction. The proof of Theorem 1.4 (1) is completed.

\subsection{Type of the lens space $\left(A_{m, n} ; m n, 0\right)$}

We verify the second term $(=m \bar{n})$ of the lens space $\left(A_{m, n} ; m n, 0\right) \cong L\left((m+n)^{2}, m \bar{n}\right)$ by the Reidemeister torsion. Here we are also interested in possibility of the transformation between the parameters $(m, n)$ and $(p, q)$.

We use the notations $\mathfrak{I}(m, n)=\left\{k_{i} \mid i=0,1, \ldots, m+n-1\right\}, u_{j}(j=0,1, \ldots, n)$ and $w_{j}(j=0,1, \ldots, m)$ defined in Section 3 .

\section{Lemma 5.5}

(1) We set $k_{i}^{\prime}=k_{i}(m+n)-i m n(i=0,1, \ldots, m+n-1)$. Then we have $k_{i}^{\prime}+k_{m+n-1-i}^{\prime}=m n$ and $k_{0}^{\prime}=0 \leq k_{i}^{\prime} \leq k_{m+n-1}^{\prime}=m n$.

(2) $\mathfrak{I}(m, n)=\left\{k_{i}^{\prime} \mid i=0,1, \ldots, m+n-1\right\}$.

Proof (1) By the definition of $k_{i}^{\prime}$ and Proposition 3.8 (1), we have

$$
\begin{aligned}
k_{i}^{\prime}+k_{m+n-1}^{\prime} & =k_{i}(m+n)-i m n+k_{m+n-1-i}(m+n)-(m+n-1-i) m n \\
& =\left(k_{i}+k_{m+n-1-i}\right)(m+n)-(m+n-1) m n \\
& =m n(m+n)-(m+n-1) m n=m n
\end{aligned}
$$

By Proposition $3.8(2)$, every element $k_{i} \in \mathfrak{I}(m, n)$ is uniquely expressed of the form $k_{u_{j}}$ or $k_{w_{j}}$. It is easy to see that $k_{0}^{\prime}=0$ and $k_{m+n-1}^{\prime}=m n$.

(i) The case $i=u_{j}(j=1,2, \ldots, n-1)$. Since $k_{i}=k_{u_{j}}=j m$ by Proposition 3.8 (2) and $\operatorname{gcd}(m, n)=1$, we have

$$
k_{i}^{\prime}=j m(m+n)-\left(\left[\frac{j m}{n}\right]+j\right) m n=m n\left(\frac{j m}{n}-\left[\frac{j m}{n}\right]\right)>0 .
$$


Since $k_{i}^{\prime}+k_{m+n-1}^{\prime}=m n$, we have $0<k_{i}^{\prime}<m n$.

(ii) The case $i=w_{j}(j=1,2, \ldots, m-1)$. Since $k_{i}=k_{w_{j}}=j n$ by Proposition 3.8 (2) and $\operatorname{gcd}(m, n)=1$, we have

$$
k_{i}^{\prime}=j n(m+n)-\left(\left[\frac{j n}{m}\right]+j\right) m n=m n\left(\frac{j n}{m}-\left[\frac{j n}{m}\right]\right)>0 .
$$

Since $k_{i}^{\prime}+k_{m+n-1}^{\prime}=m n$, we have $0<k_{i}^{\prime}<m n$. Therefore we have the result.

(2) (i) The case $i=u_{j}(j=1,2, \ldots, n-1)$. By the proof of (1) (i), $k_{i}^{\prime}$ is of the form $k_{i}^{\prime}=j^{\prime} m$ for some $j^{\prime} \in \mathbb{Z}$ and $j^{\prime} \equiv j m(\bmod n)$ is uniquely determined.

(ii) The case $i=w_{j}(j=1,2, \ldots, m-1)$. By the proof of (1) (ii), $k_{i}^{\prime}$ is of the form $k_{i}^{\prime}=j^{\prime} n$ for some $j^{\prime} \in \mathbb{Z}$ and $j^{\prime} \equiv j n(\bmod m)$ is uniquely determined.

From (i), (ii) and (1), the set $\left\{k_{i}^{\prime} \mid i=0,1, \ldots, m+n-1\right\}$ consists of distinct $m+n$ elements, and we have the result.

Lemma 5.6 For a coprime positive pair $(m, n)$, The Alexander polynomial $\Delta_{A_{m, n}}(t, x)$ of $A_{m, n}$ satisfies that

$$
\Delta_{A_{m, n}}\left(T^{m+n}, T^{-m n}\right) \doteq \frac{\left(T^{m n}-1\right)\left(T^{m+n}-1\right)}{\left(T^{m}-1\right)\left(T^{n}-1\right)}
$$

Proof As we remarked in Section 1, the first component $K_{1}$ of $A_{m, n}$ is the $(m, n)$-torus knot $T_{m, n}$. By Theorem 3.2 and the Torres formula (Lemma 2.7), we have

$$
\Delta_{A_{m, n}}(t, 1) \doteq \frac{t^{m+n}-1}{t-1} \cdot \Delta_{T_{m, n}}(t) \doteq \frac{\left(t^{m n}-1\right)\left(t^{m+n}-1\right)}{\left(t^{m}-1\right)\left(t^{n}-1\right)}=\sum_{i=0}^{m+n-1} t^{k_{i}}
$$

By Theorem 3.2, it holds that

$$
\Delta_{A_{m, n}}\left(T^{m+n}, T^{-m n}\right) \doteq \sum_{i=0}^{m+n-1} T^{k_{i}(m+n)-i m n}=\sum_{i=0}^{m+n-1} T^{k_{i}^{\prime}}
$$

and hence we have $\Delta_{A_{m, n}}\left(T^{m+n}, T^{-m n}\right) \doteq \Delta_{A_{m, n}}(T, 1)$ by Lemma $5.5(2)$.

Proof of $\left(A_{m, n} ; m n, 0\right) \cong L\left((m+n)^{2}, m \bar{n}\right)$

Assuming that $M=\left(A_{m, n} ; m n, 0\right)$ is a lens space $L(P, Q)$, it is clear that $P=(m+n)^{2}$ by the first homology. By Lemma 5.6 and the formulae (4.3) and (4.4), we have

$$
\begin{aligned}
\tau\left(M_{1}\right) \doteq & \Delta_{A_{m, n}}\left(T^{m+n}, T^{-m n}\right)\left(T^{m+n}-1\right)^{-1}=\frac{T^{m n}-1}{\left(T^{m}-1\right)\left(T^{n}-1\right)}, \\
& \tau_{(m+n)^{2}}^{\psi_{(M)}} \doteq\left(\zeta_{(m+n)^{2}}^{m}-1\right)^{-1}\left(\zeta_{(m+n)^{2}}^{n}-1\right)^{-1} .
\end{aligned}
$$

Because of the ambiguity of the Reidemeister torsion, the parameters of the lens space is almost decided but the orientation (at the choice \pm ) is left undecided.

$$
M \cong L\left((m+n)^{2}, \pm m \bar{n}\right)
$$


where $\bar{n}$ is taken modulo $(m+n)^{2}$.

Using the knowledge of existence of a lens space surgery $\left(A_{m, n} ; m n, 0\right) \cong L\left(p^{2}, p q-1\right)$ in Theorem 1.2, we can determine the sign of $\pm m \bar{n}$ as follows: Suppose that it is $-m \bar{n}$. Then we have $p q-1 \equiv-m \bar{n}\left(\bmod p^{2}\right)$, and

$$
p q \equiv 1-m \bar{n} \equiv \bar{n}(n-m) \quad\left(\bmod p^{2}\right) .
$$

Since $\operatorname{gcd}\left(p q, p^{2}\right)=p=m+n$ is changed to $\operatorname{gcd}\left(\bar{n}(n-m),(m+n)^{2}\right)=m+n$ (we regard $\bar{n}$ as an integral lift), which contradicts that $\operatorname{gcd}(\bar{n}, m+n)=1$ and $\operatorname{gcd}(n-m, m+n)=$ $\operatorname{gcd}(n-m, 2 m) \leq 2 m<m+n$.

\subsection{Type of the lens space $\left(A_{2,3} ; 7,0\right)$}

Suppose that $(m, n)=(2,3)$ and $\alpha / \beta=7$. Then we have

$$
\Delta_{A_{2,3}}(t, x)=1+t^{2} x+t^{3} x^{2}+t^{4} x^{3}+t^{6} x^{4}
$$

by Theorem 3.2, and (4.3) is computed as

$$
\tau\left(M_{1}\right) \doteq \Delta_{A_{2,3}}\left(T^{5}, T^{-7}\right)\left(T^{5}-1\right)^{-1}=\frac{1+T^{3}+T+T^{-1}+T^{2}}{T^{5}-1} \doteq \frac{1}{T-1} .
$$

Hence we have

$$
\tau^{\psi_{25}}(M) \doteq\left(\zeta_{25}-1\right)^{-1}\left(\zeta_{25}^{7}-1\right)^{-1}
$$

by (4.4). Assuming that $\left(A_{2,3} ; 7,0\right)$ is a lens space, only by our method of Reidemeister-Turaev torsion, we can say that the lens space is homeomorphic to $L(25,7) \cong L(25,-7)$.

By Kirby calculus in Figure 7 and 8 , we have $\left(A_{2,3} ; 7,0\right) \cong L(25,7)$. For the moves in Figure 7, see [Yam3]. By setting $\varepsilon=0$, we have $\left(A_{2,3} ; 6,0\right) \cong L(25,9)$. In the moves "f"in Figure 8, we used the formula in Figure 9 on a -2 -framed unknot. In the second $\mathrm{f}$ move, the union of two (3- and -4-framed) components are regarded $x$, and get unlinked by the positive full twist.

\section{Proof of Theorem 1.5 (Lens space surgeries along $B_{p, q}$ )}

In this section, we prove Theorem 1.5 on lens space surgeries along $B_{p, q}$.

\subsection{Alexander polynomial of $B_{p, q}$}

The goal of this subsection is:

Lemma 6.1 The Alexander polynomial of the link $B_{p, q}$ is

$$
\Delta_{B_{p, q}}(t, x) \doteq \frac{t^{p q} x^{p}-1}{t^{q} x-1} .
$$

where $t$ (and $x$, respectively) is represented by the meridian of the torus knot component $K_{1}$ (that of the unknotted component $K_{2}$ ). 


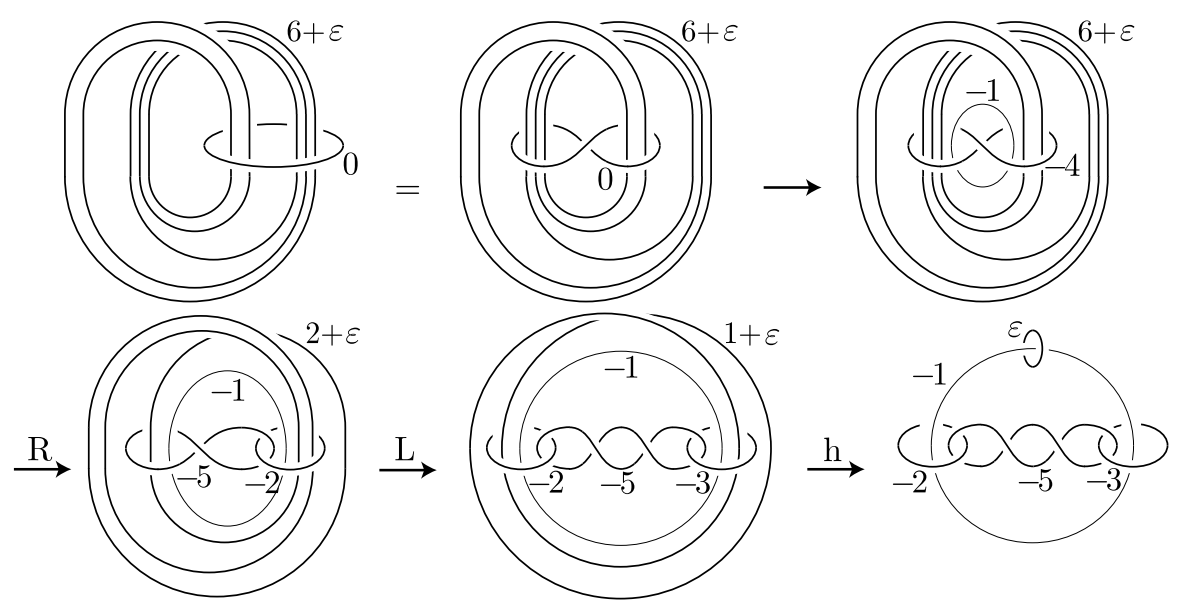

Figure $7:\left(A_{2,3} ; 7,0\right)$ is $L(25,7) \mathrm{I}$

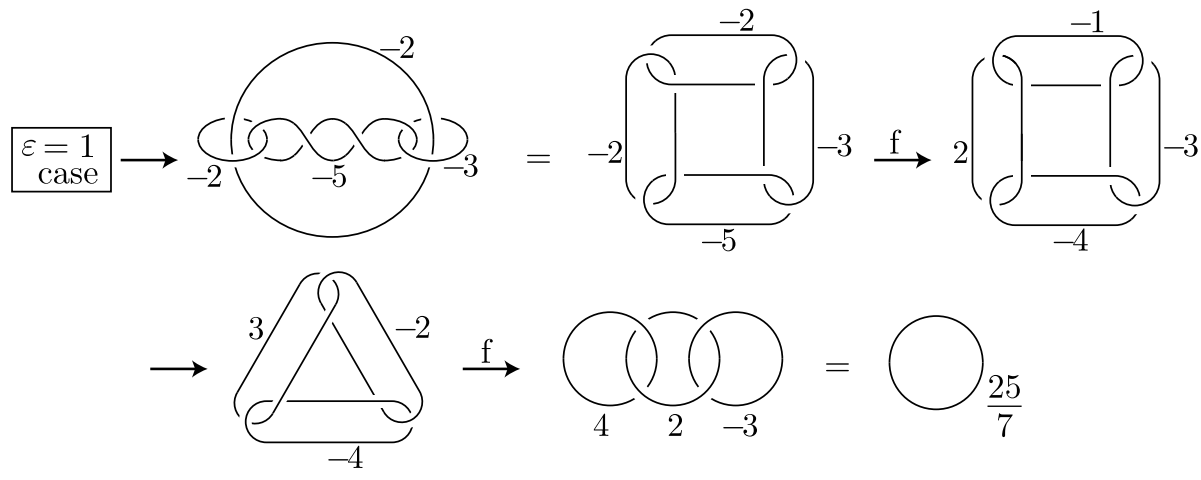

Figure 8: $\left(A_{2,3} ; 7,0\right)$ is $L(25,7)$ II

Proof We add the third component $K_{3}$ to $B_{p, q}=K_{1} \cup K_{2}$ such that $H:=K_{2} \cup K_{3}$ is a Hopf link and $K_{1}$ is isotopic to a simple closed loop in the level torus under the identification of the complement of $H$ and $T^{2} \times(-1,1)$. We set $L:=B_{p, q} \cup K_{3}$ and use the notations defined in Subsection 2.1. The complement $E_{L}$ of $L$ is homeomorphic to that $E_{\tilde{L}}$ of the connected sum $\tilde{L}=\tilde{K}_{1} \cup \tilde{K}_{2} \cup \tilde{K}_{3}$ of two Hopf links ( $\tilde{L}$ is " $B_{0,1} \cup K_{3}$ " in a sense), see Figure 10,

Our strategy of the proof is as follows: First we prove the Alexander polynomial of $L$ by studying the transformation of the homologies from the easier $\tilde{L}$ to $L$, and use the Torres formula (Lemma 2.7) on the sublink $B_{p, q}$ of $L$.

We take a homeomorphism $f: E_{\tilde{L}} \rightarrow E_{L}$ that carries each regular neighborhood of $\tilde{K}_{i}$ to that of $K_{i}(i=1,2,3)$. We will use a tilde for the notation of each component of $\tilde{L}$ : We denote the meridian-longitude system of $\tilde{K}_{i}$ by $\tilde{m}_{i}, \tilde{l}_{i}$, and its Alexander polynomial of $\tilde{L}$ by 


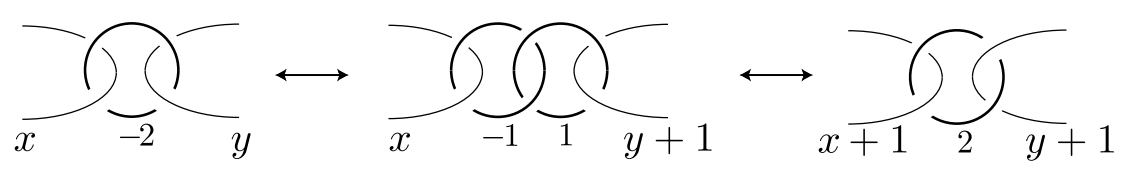

Figure 9: Kirby move $\mathrm{f}$
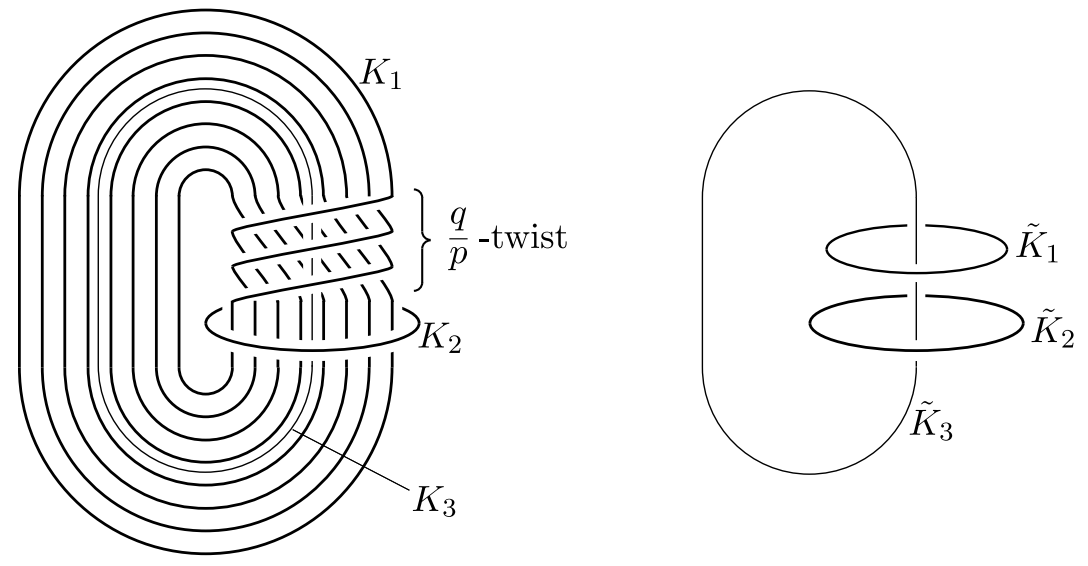

Figure 10: Link $L($ ex. $(p, q)=(8,3))$ and $\tilde{L}$

$\Delta_{\tilde{L}}\left(\tilde{t}_{1}, \tilde{t}_{2}, \tilde{t}_{3}\right)$. In $H_{1}\left(E_{L}\right)$ and $H_{1}\left(E_{\tilde{L}}\right)$, the longitudes are presented by meridians:

$$
\begin{array}{lll}
{\left[l_{1}\right]=\left[m_{2}\right]^{p}\left[m_{3}\right]^{q},} & {\left[l_{2}\right]=\left[m_{1}\right]^{p}\left[m_{3}\right],} & {\left[l_{3}\right]=\left[m_{1}\right]^{q}\left[m_{2}\right],} \\
{\left[\tilde{l}_{1}\right]=\left[\tilde{m}_{3}\right],} & {\left[\tilde{l}_{2}\right]=\left[\tilde{m}_{3}\right],} & {\left[\tilde{l}_{3}\right]=\left[\tilde{m}_{1}\right]\left[\tilde{m}_{2}\right] .}
\end{array}
$$

We note that

$$
\left[m_{2}\right]^{p}\left[l_{2}\right]^{q}=\left[m_{2}\right]^{p}\left(\left[m_{1}\right]^{p}\left[m_{3}\right]\right)^{q}=\left[m_{1}\right]^{p q}\left[l_{1}\right] .
$$

We take integers $a, b$ satisfying $a q-b p=1$. The isomorphism $f_{*}: H_{1}\left(E_{\tilde{L}}\right) \rightarrow H_{1}\left(E_{L}\right)$ induced by $f$ satisfies:

$$
\begin{array}{ll}
f_{*}\left(\left[\tilde{m}_{1}\right]\right)=\left[m_{1}\right], & f_{*}\left(\left[\tilde{l}_{1}\right]\right)=\left[m_{2}\right]^{p}\left[l_{2}\right]^{q}=\left[m_{1}\right]^{p q}\left[l_{1}\right], \\
f_{*}\left(\left[\tilde{m}_{2}\right]\right)=\left[m_{2}\right]^{a}\left[l_{2}\right]^{b}, & f_{*}\left(\left[\tilde{l}_{2}\right]\right)=\left[m_{2}\right]^{p}\left[l_{2}\right]^{q}, \\
f_{*}\left(\left[\tilde{m}_{3}\right]\right)=\left[m_{3}\right]^{q}\left[l_{3}\right]^{p}, & f_{*}\left(\left[\tilde{l}_{3}\right]\right)=\left[m_{3}\right]^{b}\left[l_{3}\right]^{a} .
\end{array}
$$

Thus, setting $t_{i}:=\left[m_{i}\right]$ and $\tilde{t}_{i}:=\left[\tilde{m}_{i}\right](i=1,2,3)$, by (6.1), (6.2) and (6.3), we have

$$
f_{*}\left(\tilde{t}_{1}\right)=t_{1}, \quad f_{*}\left(\tilde{t}_{2}\right)=t_{1}^{p b} t_{2}^{a} t_{3}^{b}, \quad f_{*}\left(\tilde{t}_{3}\right)=t_{1}^{p q} t_{2}^{p} t_{3}^{q}
$$

The Alexander polynomial of $\tilde{L}$ is known.

$$
\Delta_{\tilde{L}}\left(\tilde{t}_{1}, \tilde{t}_{2}, \tilde{t}_{3}\right) \doteq \tilde{t}_{3}-1 .
$$

Substituting (6.4) to this, we have the Alexander polynomial of $L$.

$$
\Delta_{L}\left(t_{1}, t_{2}, t_{3}\right) \doteq t_{1}^{p q} t_{2}^{p} t_{3}^{q}-1 .
$$


Hence, by Torres formula (Lemma 2.7), we have the Alexander polynomial of $B_{p, q}$ :

$$
\Delta_{B_{p, q}}(t, x) \doteq \frac{\Delta_{L}(t, x, 1)}{t^{q} x-1} \doteq \frac{t^{p q} x^{p}-1}{t^{q} x-1} .
$$

\subsection{Conditions from the first homology of $\left(B_{p, q} ; r, 0\right)$}

We set $M=\left(B_{p, q} ; \alpha / \beta, 0\right)$. The arguments are parallel to Subsection 4.1. We let $E$ denote the complement of $B_{p, q}$, and regard $M=E \cup V_{1} \cup V_{2}$, see the notations in Subsection 2.1. We set $M_{1}=E \cup V_{1}$.

We assume that $\operatorname{gcd}(p, \alpha)=1$, which is equivalent to the condition for $H_{1}(M ; \mathbb{Z})$ to be finite cyclic. Then we have $H_{1}(M ; \mathbb{Z}) \cong \mathbb{Z} / p^{2} \beta \mathbb{Z}$.

We determine the first homologies of $E, M_{1}$ and $M$, and represent some elements by the generators. First, $H_{1}(E)$ is a free abelian group of rank 2 generated by $\left[m_{1}\right]$ and $\left[m_{2}\right]$ :

$$
H_{1}(E) \cong\left\langle\left[m_{1}\right],\left[m_{2}\right] \mid-\right\rangle \cong \mathbb{Z}^{2} .
$$

We have

$$
\left[l_{1}\right]=\left[m_{2}\right]^{p} \quad \text { and } \quad\left[l_{2}\right]=\left[m_{1}\right]^{p} .
$$

We attach $V_{1}$ to $E$ to make $M_{1}$. We take integers $\gamma, \delta$ such that $\alpha \delta-\beta \gamma=-1$, and fix the meridian-longitude system $m_{1}^{\prime}, l_{1}^{\prime}$ of the solid torus $V_{1}$. In $H_{1}\left(M_{1}\right)$,

$$
\left[m_{1}^{\prime}\right]=\left[m_{1}\right]^{\alpha}\left[l_{1}\right]^{\beta}=1, \quad\left[l_{1}^{\prime}\right]=\left[m_{1}\right]^{\gamma}\left[l_{1}\right]^{\delta}
$$

and (6.5) also hold. Thus, we have

$$
\begin{aligned}
H_{1}\left(M_{1}\right) & \cong\left\langle\left[m_{1}\right],\left[m_{2}\right] \mid\left[m_{1}\right]^{\alpha}\left[m_{2}\right]^{p \beta}=1\right\rangle \\
& \cong\langle T \mid-\rangle \cong \mathbb{Z}
\end{aligned}
$$

where $T=\left[m_{1}\right]^{\gamma^{\prime}}\left[m_{2}\right]^{\delta^{\prime}}$ by taking integers $\gamma^{\prime}, \delta^{\prime}$ satisfying $\alpha \delta^{\prime}-p \beta \gamma^{\prime}=-1$. By the relations above, we have

$$
\begin{aligned}
{\left[m_{1}\right] } & =\left[m_{1}\right]^{-\alpha \delta^{\prime}+p \beta \gamma^{\prime}} \\
& =\left(\left[m_{1}\right]^{\alpha}\left[m_{2}\right]^{p \beta}\right)^{-\delta^{\prime}}\left(\left[m_{1}\right]^{\gamma^{\prime}}\left[m_{2}\right]^{\delta^{\prime}}\right)^{p \beta}=T^{p \beta} \\
{\left[m_{2}\right] } & =\left[m_{2}\right]^{-\alpha \delta^{\prime}+p \beta \gamma^{\prime}} \\
& =\left(\left[m_{1}\right]^{\alpha}\left[m_{2}\right]^{p \beta}\right)^{\gamma^{\prime}}\left(\left[m_{1}\right]^{\gamma^{\prime}}\left[m_{2}\right]^{\delta^{\prime}}\right)^{-\alpha}=T^{-\alpha} \\
{\left[l_{1}^{\prime}\right] } & =\left[m_{1}\right]^{\gamma}\left[l_{1}\right]^{\delta} \\
& =\left[m_{1}\right]^{\gamma}\left[m_{2}\right]^{p \delta}=T^{p \beta \gamma-p \alpha \delta}=T^{p} .
\end{aligned}
$$

Next, we attach $V_{2}$ to $M_{1}$ to make $M$. By (6.5) and (6.6) in $H_{1}(M)$, we have

$$
\left[m_{2}^{\prime}\right]=\left[l_{2}\right]=\left[m_{1}\right]^{p}=T^{p^{2} \beta}=1, \quad\left[l_{2}^{\prime}\right]=\left[m_{2}\right]=T^{-\alpha},
$$

and

$$
H_{1}(M) \cong\left\langle T \mid T^{p^{2} \beta}=1\right\rangle \cong \mathbb{Z} / p^{2} \beta \mathbb{Z}
$$




\subsection{Proof of Theorem 1.5}

By Surgery formula II (Lemma 2.5) (2), Lemma 6.1 and (6.6), we have

$$
\tau\left(M_{1}\right) \doteq \Delta_{B_{p, q}}\left(T^{p \beta}, T^{-\alpha}\right)\left(T^{p}-1\right)^{-1} \doteq \frac{T^{p(-\alpha+p q \beta)}-1}{\left(T^{-\alpha+p q \beta}-1\right)\left(T^{p}-1\right)}
$$

Let $\iota: \mathbb{Z}\left[H_{1}\left(M_{1}\right)\right] \rightarrow \mathbb{Z}\left[H_{1}(M)\right]$ be a ring homomorphism induced from the natural inclusion, $\psi_{d}: \mathbb{Z}\left[H_{1}(M)\right] \rightarrow \mathbb{Q}\left(\zeta_{d}\right)$ a ring homomorphism such that $\psi_{d}(T)=\zeta_{d}$, and set $\rho_{d}:=\psi_{d} \circ \iota$, where $d$ is a divisor of $p$. Note that $\operatorname{gcd}(d, \alpha)=1$. Then by Surgery formula I (Lemma 2.3), (6.6) and (6.7), we have

$$
\tau^{\psi_{d}}(M) \doteq \tau^{\rho_{d}}\left(M_{1}\right)\left(\zeta_{d}^{\alpha}-1\right)^{-1} \doteq(\alpha-p q \beta)\left(\zeta_{d}^{\alpha}-1\right)^{-2}
$$

Suppose that $M$ is a lens space. Then its Reidemeister torsion is equal to that of a lens space (Example 2.6), i.e. there exist integers $i$ and $j$ such that $\operatorname{gcd}(p, i)=\operatorname{gcd}(p, j)=1$ and

$$
(\alpha-p q \beta)\left(\zeta_{d}^{\alpha}-1\right)^{-2} \doteq\left(\zeta_{d}^{i}-1\right)^{-1}\left(\zeta_{d}^{j}-1\right)^{-1}
$$

By taking $d$-norm (see Subsection 2.2) of both hand-sides, we have a necessary condition

$$
|\alpha-p q \beta|=1
$$

by Lemma 2.9 .

Conversely, suppose $|\alpha-p q \beta|=1$ and set $\varepsilon=\alpha-p q \beta(= \pm 1)$. We can prove that $\left(B_{p, q} ; \alpha / \beta, 0\right)$ is homeomorphic to the lens space by Kirby-Rolfsen moves [Ro] as follows (cf. [Mos, see also [IS, Proposition 4.1]): $M$ is the result of $(\alpha / \beta, 0, \infty)$-surgery along $L=$ $B_{p, q} \cup K_{3}$. It is homeomorphic to that of $(\varepsilon / \beta,-p / a,-a / p)$-surgery $(a q-b p=1)$ along $\tilde{L}$ because of the identification between $E_{L}$ and $E_{\tilde{L}}$ in $(6.3)$, and that of $(-p / a,-(a+\varepsilon p \beta) / p)$ surgery along the Hopf link by $(-\varepsilon \beta)$-twist on $\tilde{K}_{1}$. Therefore we have $M \cong L\left(p^{2} \beta, \alpha\right)$.

\subsection{Other surgeries along $B_{p, q}$}

The link $B_{p, q}$ is included in a family of Burde-Murasugi's links [BM], whose complements admit structures of Seifert fiber spaces. The complement of $B_{p, q}$ is homeomorphic to that of the $(3,3)$-torus link (and to that of the link in Figure 10 also). Thus we can determine all Dehn surgeries along $B_{p, q}$ by a result of the first author and M. Shimozawa [KS] on Dehn surgeries along torus links.

Let $\left(B_{p, q} ; \alpha_{1} / \beta_{1}, \alpha_{2} / \beta_{2}\right)$ be the result of $\left(\alpha_{1} / \beta_{1}, \alpha_{2} / \beta_{2}\right)$-surgery along $B_{p, q}$ where $\alpha_{i}$ and $\beta_{i}(i=1,2)$ are coprime integers with $\beta_{i} \geq 1$.

Theorem 6.2 We set $\varepsilon_{1}=\alpha_{1}-\beta_{1} p q, \varepsilon_{2}=\alpha_{2} q-\beta_{2} p, P=\alpha_{1} \alpha_{2}-\beta_{1} \beta_{2} p^{2}$, and $a$ and $b$ as coprime integers such that $a q-b p=1$. Then the result of surgery $M=\left(B_{p, q} ; \alpha_{1} / \beta_{1}, \alpha_{2} / \beta_{2}\right)$ with $p \geq 2$ is as follows:

(1) If $\varepsilon_{1} \varepsilon_{2} \neq 0$, then $M$ is a Seifert fiber space over $S^{2}$ with at most three singular fibers whose multiplicities are $\left|\varepsilon_{1}\right|,\left|\varepsilon_{2}\right|$ and $p$. 
(2) Suppose $\varepsilon_{1} \varepsilon_{2} \neq 0$. Then $M$ is a lens space if and only if $\left|\varepsilon_{1}\right|=1$ or $\left|\varepsilon_{2}\right|=1$. The resulting lens spaces are

$$
L\left(P,-\beta_{2} \varepsilon_{1}-\beta_{1} \varepsilon_{2} q\right) \cong L\left(P,-\alpha_{1} \beta_{2}-\alpha_{2} \beta_{1} q^{2}+2 \beta_{1} \beta_{2} p q\right)
$$

in both cases.

(i) If $\varepsilon_{1}=0$ (i.e. $\alpha_{1} / \beta_{1}=p q$ ), then $M$ is a connected sum of lens spaces

$$
L\left(\varepsilon_{2}, \alpha_{2} b-\beta_{2} a\right) \sharp L(p,-q) .
$$

(ii) If $\varepsilon_{2}=0$ (i.e. $\alpha_{2} / \beta_{2}=p / q$ ), then $M$ is a connected sum of lens spaces

$$
L\left(\varepsilon_{1},-\beta_{1}\right) \sharp L(p,-q) .
$$

Here we regard $L( \pm 1, Q)\left(\cong S^{3}\right)$ and $L(0, Q)\left(\cong S^{1} \times S^{2}\right)$ for any $Q$ as lens spaces.

Proof Let $L_{p, q}$ be the link $L$ in the proof of Lemma 6.1. Then $L_{1,1}$ is the (3,3)-torus link. We set $L_{1,1}=\hat{L}=\hat{K}_{1} \cup \hat{K}_{2} \cup \hat{K}_{3}$. Let $\hat{m}_{i}$ and $\hat{l}_{i}$ be a meridian and a longitude of $\hat{K}_{i}(i=1,2,3)$, respectively.

Let $h: E_{L} \rightarrow E_{\hat{L}}$ be a homeomorphism inducing an isomorphism $h_{*}: H_{1}\left(E_{L}\right) \rightarrow H_{1}\left(E_{\hat{L}}\right)$ such that

$$
\begin{aligned}
h_{*}\left(\left[m_{1}\right]\right) & =\left[\hat{m}_{1}\right], & & h_{*}\left(\left[l_{1}\right]\right)=\left[\hat{m}_{1}\right]^{-p q+1}\left[\hat{l}_{1}\right], \\
h_{*}\left(\left[m_{2}\right]\right) & =\left[\hat{m}_{2}\right]^{q-b}\left[\hat{l}_{2}\right]^{-b}, & & h_{*}\left(\left[l_{2}\right]\right)=\left[\hat{m}_{2}\right]^{-p+a}\left[\hat{l}_{2}\right]^{a}, \\
h_{*}\left(\left[m_{3}\right]\right) & =\left[\hat{m}_{3}\right]^{a}\left[\hat{l}_{3}\right]^{-p+a}, & & h_{*}\left(\left[l_{3}\right]\right)=\left[\hat{m}_{3}\right]^{-b}\left[\hat{l}_{3}\right]^{q-b} .
\end{aligned}
$$

By the relations, we have

$$
\begin{aligned}
h_{*}\left(\left[m_{1}\right]^{\alpha_{1}}\left[l_{1}\right]^{\beta_{1}}\right) & =\left[\hat{m}_{1}\right]^{\alpha_{1}-\beta_{1}(p q-1)}\left[\hat{l}_{1}\right]^{\beta_{1}}, \\
h_{*}\left(\left[m_{2}\right]^{\alpha_{2}}\left[l_{2}\right]^{\beta_{2}}\right) & =\left[\hat{m}_{2}\right]^{\alpha_{2}(q-b)-\beta_{2}(p-a)}\left[\hat{l}_{2}\right]^{-\alpha_{2} b+\beta_{2} a}, \\
h_{*}\left(\left[m_{3}\right]\right) & =\left[\hat{m}_{3}\right]^{a}\left[\hat{l}_{3}\right]^{-p+a},
\end{aligned}
$$

and hence $M$ is the result of $\left(\left\{\alpha_{1}-\beta_{1}(p q-1)\right\} / \beta_{1},\left\{\alpha_{2}(q-b)-\beta_{2}(p-a)\right\} /\left(-\alpha_{2} b+\beta_{2} a\right), a /(-p+\right.$ a))-surgery along the $(3,3)$-torus link. By [KS, Theorem $3.3(3)]$, if $\varepsilon_{1} \varepsilon_{2} \neq 0$, then $M$ is a Seifert fiber space over $S^{2}$ with at most three singular fibers whose indices are

$$
\left(-1 ; \frac{\beta_{1}}{\varepsilon_{1}}, \frac{-\alpha_{2} b+\beta_{2} a}{\varepsilon_{2}}, \frac{-p+a}{p}\right)=\left(0 ; \frac{\beta_{1}}{\varepsilon_{1}}, \frac{-\alpha_{2} b+\beta_{2} a}{\varepsilon_{2}}, \frac{a}{p}\right),
$$

see Figure 11, Other cases are obtained from it.

Remark 6.3 The convention on lens spaces in [KS is different from that in the present paper. Theorem 1.5 corresponds to the case $\alpha_{2}=0$ and $\beta_{2}=1$ in Theorem 6.2 (2), and we can deduce Theorem 1.5 (2) from Theorem 6.2 (2) after some deformations.

\section{Lens space surgeries along $A_{m, n}$ other than $(r, 0)$-surgery}

Contrasted to the case of $B_{p, q}$ in Subsection 6.4, to determine all Dehn surgeries along $A_{m, n}$ is a hard problem. Related to Conjecture 1.11, in Subsection 7.1 and Subsection 7.2, we compute the Reidemeister torsions of $M$ under the case (1) $\alpha_{1} / \beta_{1}=m n$ and (2) $\alpha_{1} / \beta_{1}=7$ for $(m, n)=(2,3)$, respectively. 


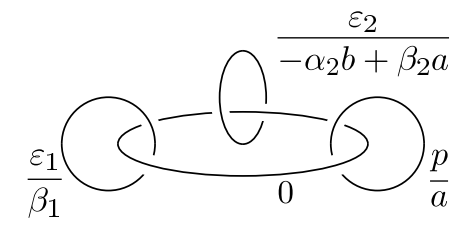

Figure 11: Seifert fiber space $\left(B_{p, q} ; \alpha_{1} / \beta_{1}, \alpha_{2} / \beta_{2}\right)$

\subsection{The case $\left(A_{m, n} ; m n, r\right)$}

For $A_{m, n}=K_{1} \cup K_{2}$, we compute the Reidemeister torsions of $M=\left(A_{m, n} ; m n, r\right)$. We set $r=\alpha / \beta$, where $\alpha$ and $\beta$ are coprime integers with $\beta \geq 1$, and $P=(m+n)^{2} \beta-m n \alpha$. Let $d(\geq 2)$ be a divisor of $P$.

We use the notations $E, M_{1}$ and $M$ defined in Subsection 2.1. By the similar way to Subsection 4.1, we have two lemmas.

Lemma 7.1 We take integers $u$ and $v$ as $(m+n) u-m n v=1$, and set $T=\left[m_{1}\right]^{u}\left[m_{2}\right]^{v}$ in $H_{1}\left(M_{1}\right)$. Then

(1) $T$ is a generator of $H_{1}\left(M_{1}\right)$.

(2) It holds that $\left[m_{1}\right]=T^{m+n}$ and $\left[m_{2}\right]=T^{-m n}$.

Lemma 7.2 Let $T$ be the generator of $H_{1}(M)$ which is induced by that of $H_{1}\left(M_{1}\right)$ in Lemma 7.1. Let $\psi_{d}: \mathbb{Z}\left[H_{1}(M)\right] \rightarrow \mathbb{Q}\left(\zeta_{d}\right)$ be a ring homomorphism such that $\psi_{d}(T)=\zeta_{d}$. Then the core of $V_{2}$ (i.e., $\left.\left[l_{2}\right]\right)$ is mapped to $\zeta_{d}^{(m+n)^{2} \delta-m n \gamma}$ by $\psi_{d}$, where $\gamma$ and $\delta$ are integers such that $\alpha \delta-\beta \gamma=-1$.

Lemma 7.3 The integer $(m+n)^{2} \delta-m n \gamma$ is coprime to $P$.

Proof We have

$$
\left(\begin{array}{ll}
\alpha & \beta \\
\gamma & \delta
\end{array}\right)\left(\begin{array}{c}
-m n \\
(m+n)^{2}
\end{array}\right)=\left(\begin{array}{c}
(m+n)^{2} \beta-m n \alpha \\
(m+n)^{2} \delta-m n \gamma
\end{array}\right)
$$

Since $\alpha \delta-\beta \gamma=-1$, the matrix is invertible over $\mathbb{Z}$, thus we have

$$
\operatorname{gcd}\left((m+n)^{2} \beta-m n \alpha,(m+n)^{2} \delta-m n \gamma\right)=\operatorname{gcd}\left((m+n)^{2}, m n\right)=1 .
$$

Theorem 7.4

$$
\tau^{\psi_{d}}(M) \doteq \frac{\zeta_{d}^{m n}-1}{\left(\zeta_{d}^{m}-1\right)\left(\zeta_{d}^{n}-1\right)\left(\zeta_{d}^{(m+n)^{2} \delta-m n \gamma}-1\right)}
$$

Proof We use the surgery formula II (Lemma 2.5). By Lemma 7.2 and Lemma 7.3, $\zeta_{d}^{(m+n)^{2} \delta-m n \gamma}$ is a primitive $d$-th root of unity. By the Alexander polynomial of $A_{m, n}$ in Theorem 3.2, Lemma 7.1, Lemma 7.2 and Lemma 5.6, we have the result. 
By the Franz lemma (Lemma 2.10), $\tau^{\psi_{d}}(M)$ is same with the Reidemeister torsion of a lens space if and only if

$$
m \equiv \pm 1, \quad n \equiv \pm 1 \quad \text { or } \quad(m+n)^{2} \delta-m n \gamma \equiv \pm m n \quad(\bmod P)
$$

\section{Lemma 7.5}

(i) If $\beta=1$, then $M$ has the same Reidemeister torsions with a lens space $L(P, \pm m \bar{n})$ where $n \bar{n} \equiv 1(\bmod P)$.

(ii) $(m+n)^{2} \delta-m n \gamma \equiv \pm m n(\bmod P)$ is equivalent to $\beta \equiv \pm 1(\bmod P)$.

(iii) Suppose that $\alpha<0$, and $M$ has the same Reidemeister torsions with a lens space. Then we have $\beta=1$.

Proof (i) If $\beta=1$, then we can take $\gamma=1, \delta=0$, thus $(m+n)^{2} \delta-m n \gamma \equiv \pm m n(\bmod P)$.

(ii) Suppose that $(m+n)^{2} \delta-m n \gamma \equiv \pm m n(\bmod P)$. Then by (7.1), it holds that

$$
\left(\begin{array}{c}
-m n \\
(m+n)^{2}
\end{array}\right) \equiv\left(\begin{array}{cc}
-\delta & \beta \\
\gamma & -\alpha
\end{array}\right)\left(\begin{array}{c}
0 \\
\pm m n
\end{array}\right)=\left(\begin{array}{l} 
\pm \beta m n \\
\mp \alpha m n
\end{array}\right) \quad(\bmod P)
$$

hence $\beta \equiv \pm 1(\bmod P)$. Conversely suppose that $\beta \equiv \pm 1(\bmod P)$. Then we can take $\gamma \equiv \pm 1, \delta \equiv 0(\bmod P)$, thus $(m+n)^{2} \delta-m n \gamma \equiv \pm m n(\bmod P)$.

(iii) $P=(m+n)^{2} \beta-m n \alpha=(m+n)^{2} \beta+m n|\alpha|$. By the assumption $2 \leq m<n$, it holds that $m \not \equiv \pm 1$ and $n \not \equiv \pm 1(\bmod P)$. Since $0<\beta<P-m n|\alpha|$, we have $\beta=1$.

Extending the Kirby calculus in [Yam3], one can prove the following:

Lemma 7.6 (An extension of [Yam3]) The surgered manifold $\left(A_{m, n} ; m n, \alpha / \beta\right)$ is a Seifert fiber space over $S^{2}$ with at most three singular fibers whose indices are

$$
\left(-2 ; \frac{n}{m}, \frac{m}{n},-\frac{\alpha}{\beta}\right)
$$

see Figure 12.

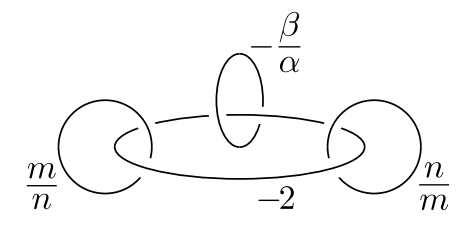

Figure 12: Seifert manifold $\left(A_{m, n} ; m n, \alpha / \beta\right)$

As a corollary, we can prove some lens space surgeries.

Corollary 7.7 The surgered manifold $\left(A_{m, n} ; m n, \alpha / \beta\right)$ is a lens space if and only if $\beta=1$ (thus $\left.P=(m+n)^{2}-\alpha m n\right)$ and the lens space is $L(P, m \bar{n})$, where $n \bar{n} \equiv 1(\bmod P)$. 
Proof (Using Lemma [7.6) We apply [KS, Lemma 2.2] to the description in Figure 12 in the case $\beta=1$. Then the manifold is $L(P, Q)$ with

$$
P=(m+n)^{2}-\alpha m n, \quad Q=m x+\{m(\alpha-2)-n\} y,
$$

where $x, y$ are coprime integers satisfying $n x+m y=1$, and it holds that

$$
\begin{aligned}
n Q & =m n x+\left\{m n(\alpha-2)-n^{2}\right\} y \\
& \equiv m n x+\left\{(m+n)^{2}-2 m n-n^{2}\right\} y(\bmod P) \\
& =m n x+m^{2} y \\
& =m(n x+m y) \\
& =m .
\end{aligned}
$$

\subsection{The case $\left(A_{2,3} ; 7, r\right)$}

We set $(m, n)=(2,3), r=\alpha / \beta$, where $\alpha$ and $\beta$ are coprime integers with $\beta \geq 1$ and $P=25 \beta-7 \alpha$. Let $d(\geq 2)$ be a divisor of $P$. We compute the Reidemeister torsions of $M=\left(A_{2,3} ; 7, r\right)$.

Theorem 7.8 Let $\psi_{d}: \mathbb{Z}\left[H_{1}(M)\right] \rightarrow \mathbb{Q}\left(\zeta_{d}\right)$ be a ring homomorphism which maps a generator of $H_{1}(M)$ to $\zeta_{d}$. Then we have

$$
\tau^{\psi_{d}}(M) \doteq\left(\zeta_{d}-1\right)^{-1}\left(\zeta_{d}^{7 \gamma-25 \delta}-1\right)^{-1}
$$

where integers $\gamma$ and $\delta$ satisfy $\alpha \delta-\beta \gamma=-1$.

In particular, $M$ has the same Reidemeister torsions with a lens space $L(P, \pm(7 \gamma-25 \delta))$.

We can verify the lens space surgeries.

Lemma 7.9 The surgered manifold $\left(A_{2,3} ; 7, \alpha / \beta\right)$ is a lens space $L(25 \beta-7 \alpha, 2 \alpha-7 \beta)$.

Proof We modify the Kirby calculus in Figure 7 and Figure 8 as in Figure 13 ,

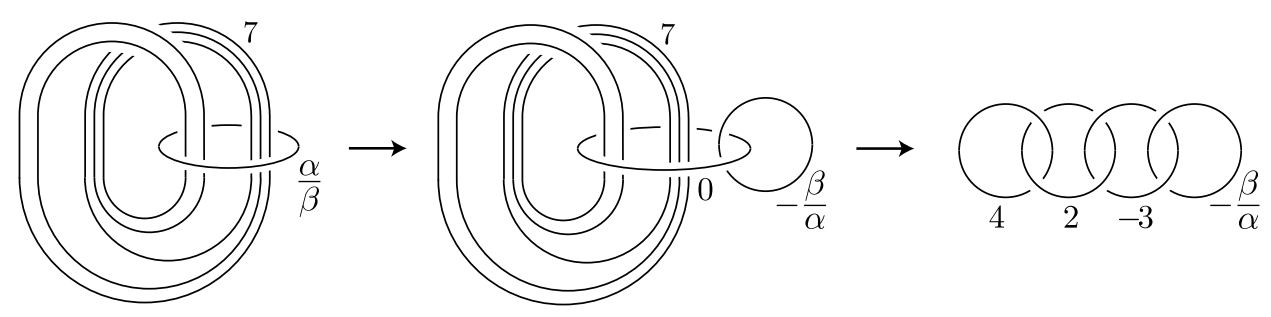

Figure 13: Lens space $\left(A_{2,3} ; 7, \alpha / \beta\right)$

\section{Final remarks}

In this section, we give some remarks concerning our results. 


\subsection{Alternative proof for Theorem 1.4 (1)}

At the beginning of our study, the authors did not know whether $\left(A_{m, n} ; \emptyset, 0\right)$ is proved to be non-Seifert, and our method by Reidemeister-Turaev torsion works without the knowledge. In fact, we are interested in the condition on the Alexander polynomial for a link to admit a lens space surgery.

In the preparation of this paper, K. Motegi informed us that $\left(A_{m, n} ; \emptyset, 0\right)$ is proved to be non-Seifert. We give an alternative rough proof of Theorem $1.4(1)$ by assuming $\left(A_{m, n} ; \emptyset, 0\right)$ is non-Seifert and by using the Cyclic Surgery Theorem [CGLS].

Proof of Theorem 1.4 (1) (Using the results in [DMM1] and [CGLS]) We set $M=$ $\left(A_{m, n} ; r, 0\right)(r \in \mathbb{Q})$. By the Cyclic Surgery Theorem [CGLS], only $r=m n-1$ or $m n+1$ can be a solution of Problem 1.1 other than $r=m n$.

Case $1 \quad r=m n-1$

This case does not occur by Lemma 4.5 (2).

Case $2 r=m n+1$

By Lemma 4.3, we have

$$
\tau^{\psi_{d}^{\prime}}(M) \doteq\left(\xi^{m-1}-1\right)\left(\xi^{m+1}-1\right)(\xi-1)^{-2}\left(\xi^{m}-1\right)^{-2}
$$

where $\xi$ is a primitive $d$-th root of unity $(d \mid m+n$ and $d \geq 2)$. If $M=\left(A_{m, n} ; m n+1,0\right)$ is a lens space, there exists integers $i$ and $j \operatorname{such}$ that $\operatorname{gcd}(i, m+n)=\operatorname{gcd}(j, m+n)=1$, as in (4.8):

$$
\left(\xi^{m-1}-1\right)\left(\xi^{m+1}-1\right)\left(\xi^{i}-1\right)\left(\xi^{j}-1\right) \doteq(\xi-1)^{2}\left(\xi^{m}-1\right)^{2} .
$$

By the similar way to the proof of Lemma 4.10, using Franz lemma [Fz] (Lemma 2.10), we have

$$
\{ \pm(m-1), \pm(m+1), \pm i, \pm j(\bmod m+n)\}=\{ \pm 1, \pm 1, \pm m, \pm m(\bmod m+n)\}
$$

as multiple sets. It has a unique solution $(m, n)=(2,3)$ with $(i, j)=(1,3)$. Thus we have $r=7$.

\subsection{Algebraic generalization}

Our main theorems can be extended to the cases of 2-component links in homology 3-spheres with the same Alexander polynomials as $A_{m, n}$ and $B_{p, q}$.

Theorem 8.1 Let $L_{m, n}$ be a 2-component link in a homology 3-sphere with the same Alexander polynomial as $A_{m, n}$ (Theorem 3.2). If $\left(L_{m, n} ; r, 0\right)$ is a lens space, then we have $r=m n$, or $r=7$ for $(m, n)=(2,3)$. Moreover we have $\left(L_{m, n} ; m n, 0\right) \cong L\left((m+n)^{2}, \pm m \bar{n}\right)$ or $\left(L_{2,3} ; 7,0\right) \cong L(25,7)$ respectively, where $n \bar{n} \equiv 1\left(\bmod (m+n)^{2}\right)$.

Theorem 8.2 Let $L_{p, q}^{\prime}$ be a 2-component link in a homology 3-sphere with the same Alexander polynomial as $B_{p, q}\left(\right.$ Lemma 6.1). If $\left(L_{p, q}^{\prime} ; \alpha / \beta, 0\right)$ is a lens space, then we have $|\alpha-p q \beta|=$ 1. Furthermore, in this case, we have $\left(L_{p, q}^{\prime} ; \alpha / \beta, 0\right) \cong L\left(p^{2} \beta, \pm \alpha\right)$.

We remark that the converses of the theorems above do not always hold in general.

Acknowledgement. The authors would like to thank to Professor Kimihiko Motegi and Professor Takayuki Morifuji for thier useful advices. 


\section{References}

[Ak] S. Akbulut, On 2-dimensional homology classes of 4-manifolds, Math. Proc. Camb. Phil. Soc. 82 (1977), 99-106.

[Ba] K. Baker, Knots on once-punctured torus fibers, dissertation, The University of Texas Austin (2004).

[Ber] J. Berge, Some knots with surgeries yielding lens spaces, (Unpublished manuscript, 1990).

[Bir] J. S. Birman, Braids, links, and mapping class groups, Annals of Mathematics Studies, No. 82. Princeton University Press, Princeton, N.J.; University of Tokyo Press, Tokyo, 1974.

[BL] S. Boyer and D. Lines, Surgery formulae for Casson's invariant and extensions to homology lens spaces, J. Reine Angew. Math. 45 (1990), 181-220.

[BM] G. Burde and K. Murasugi, Surgery formulae for Casson's invariant and extensions to homology lens spaces, Duke Math. J. 37 (1970), 89-93.

[CGLS] M. Culler, M. Gordon, J. Luecke and P. Shalen, Dehn surgery on knots, Ann. of Math. 125 (1987), 237-300.

$[\mathrm{CH}]$ A. Casson and J. Harer, Some homology lens spaces which bound rational homology balls, Pacific J. Math. 96 (1981), no. 1, 23-36.

[DMM1] A. Deruelle, K. Miyazaki and K. Motegi, Networking Seifert surgeries on knots II: The Berge's lens surgeries, Topol. Appl. 156 (2009), no. 6, 1083-1113.

[DMM2] A. Deruelle, K. Miyazaki and K. Motegi, Networking Seifert surgeries on knots, Mem. Amer. Math. Soc. 217 (2012).

[EMM] H. Endo, T. Mark and J. Horn-Morris, Monodromy substitutions and rational blowdowns, J. Topology 4 (2011), no.1, 227-253.

[FS] R. Fintushel and R. Stern, Rational blow downs of smooth 4-manifolds, J. Differential Geom. 46 (1997), no. 2, 181-235.

[Fz] W. Franz, Über die Torsion einer Überdeckung, J. Reine Angew. Math. 173 (1935), 245-254.

[GS] R. Gompf and A. Stipsicz, 4-manifolds and Kirby calculus, Grad.Studies in Math. 20 A.M.S. Providence, RI (1999).

[Go] C. McA. Gordon, Dehn surgery and satellite knots, Trans. Amer. Math. Soc. 275 (1983), no.2, $687-708$.

[IS] K. Ichihara and T. Saito, Surgical distance between lens spaces, Tokyo J. Math. 34 (2011), no.1, 153-164. Preprint version, arXiv: math.GT/0809.3707.

[Kad1] T. Kadokami, Reidemeister torsion and lens surgeries on knots in homology 3-spheres I, Osaka J. Math. 43 (2006), no.4, 823-837.

[Kad2] T. Kadokami, Reidemeister torsion of Seifert fibered homology lens spaces and Dehn surgery, Algebr. Geom. Topol. 7 (2007), 1509-1529.

[Kad3] T. Kadokami, Reidemeister torsion and lens surgeries on knots in homology 3-spheres II, Top. Appl. 155 (2008), no.15, 1699-1707.

[Kad4] T. Kadokami, Hyperbolicity and identification of Berge knots of types VII and VIII, arXiv math.GT/1107.0379.

[KS] T. Kadokami and M. Shimozawa, Dehn surgery along torus links, J. Knot Theory Ramifications 19 (2010), no.4, 489-502. 
[KMS] T. Kadokami, N. Maruyama and M. Shimozawa, Lens surgeries along the $n$-twisted Whitehead link, to appear in Kyungpook Mathematical Journal.

[KY1] T. Kadokami and Y. Yamada, Reidemeister torsion and lens surgeries on $(-2, m, n)$-pretzel knots, Kobe J. Math. 23 (2006), no.1-2, 65-78.

[KY2] T. Kadokami and Y. Yamada, A deformation of the Alexander polynomials of knots yielding lens spaces, Bull. of Austral. Math. Soc. 75 (2007), no.1, 75-89.

[Kaw] A. Kawauchi, A Survay of Knot Theory, Translated and revised from the 1990 Japanese original by the author. Birkhäuser Verlag, Basel, 1996.

[Kir] R. Kirby, A calculus for framed links in $S^{3}$, Invent. Math. 45 (1978), no.1, 35-56.

[Li] P. Lisca, On symplectic fillings of lens spaces, Trans. Amer. Math. Soc. 360 (2008), no. 2, 765-799.

[Mil] J. W. Milnor, A duality theorem for Reidemeister torsion, Ann. of Math. (2), 76 (1962), 137-147.

[Mos] L. Moser, Elementary surgery along a torus knot, Pacific J. Math. 38 (1971), 737-745.

[Pa] J. Park, Seiberg-Witten invariants of generalised rational blow-downs, Bull. Austral. Math. Soc. 56 (1997), no. 3, 363-384.

[Ro] D. Rolfsen, Rational surgery calculus: extension of Kirby's theorem, Pacific J. Math. 110 (1984), $377-386$.

[Sa] T. Sakai, Reidemeister torsion of a homology lens space, Kobe J. Math. 1 (1984), no.1, 47-50.

[SSW] A. I. Stipsicz, Z. Szabo and J. Wahl, Rational blow downs and smoothings of surface singularities, Journal of Topology 1 (2008), no.2, 477-517.

[Tor] G. Torres, On the Alexander polynomial, Ann. of Math. (2), 57 (1953), 57-89.

[Tur1] V. G. Turaev, Reidemeister torsion in knot theory, Russian Math. Surveys 41-1 (1986), 119182.

[Tur2] V. G. Turaev, Introduction to Combinatorial Torsions, Lectures in Math. ETH Zürich, Birkhüser Verlag, Basel (2001).

[Was] L. C. Washington, Introduction to Cyclotomic Fields, Graduate Texts in Mathematics 83, Springer-Verlag, New York (1982).

[Yam1] Y. Yamada, Berge's knots in the fiber surfaces of genus one, lens spaces and framed links, J. Knot Theory Ramifications 14 (2005), no.2, 177-188.

[Yam2] Y. Yamada, Lens space surgeries as A'Campo's divide knots, Algebr. Geom. Topol. 9 (2009), 397-428.

[Yam3] Y. Yamada, Generalized rational blow-down, torus knots and Euclidean algorithm, arXiv math.GT/0708.2316.

[Yam4] Y. Yamada, Canonical forms of the knots in the genus one fiber surfaces, Bulletin of the University of Electro-Communications 22-1 (2010), 25-31.

Teruhisa KADOKAMI

Department of Mathematics, East China Normal University,

Dongchuan-lu 500, Shanghai, 200241, China

kadokami2007@yahoo.co.jp, mshj@math.ecnu.edu.cn

Yuichi YAMADA

Department of Mathematics, The University of Electro-Communications

1-5-1,Chofugaoka, Chofu, Tokyo, 182-8585, JAPAN

yyyamada@sugaku.e-one.uec.ac.jp 